\title{
ANION EXCHANGE AT THE INTERFACES OF MIXED ANION III-V HETEROSTRUCTURES GROWN BY MOLECULAR BEAM EPITAXY
}

\author{
A Dissertation \\ Presented to \\ The Academic Faculty \\ By \\ Terence D. Brown
}

\begin{abstract}
In Partial Fulfillment
Of the Requirements for the Degree

Doctor of Philosophy in Electrical and Computer Engineering
\end{abstract}

Georgia Institute of Technology

December 2003 


\section{ANION EXCHANGE AT THE INTERFACES OF MIXED ANION III-V HETEROSTRUCTURES GROWN BY MOLECULAR BEAM EPITAXY}

Approved by:

Dr. April S. Brown, Advisor

Dr. Gary S. May, Advisor

Dr. Stephen E. Ralph

Dr. William A. Doolittle

Dr. Zhong Lin Wang

Date Approved _ 11/24/2003 


\section{Dedicated to}

My Loving Mother Shirley,

Sisters Kimberly and Brittani,

Brothers Craig and Eric

... Endless Support 


\section{ACKNOWLEDGEMENT}

I would like to express my deepest appreciation to Dr. Gary S. May and Dr. April S. Brown for serving as my thesis advisors, providing valuable guidance and advice, innumerable hours spent reviewing my thesis, their patience and understanding, and unyielding support and encouragement throughout the course of my graduate studies. I would also like to thank Dr. William A. Doolittle for providing valuable discussions regarding molecular beam epitaxy, sharing innovative ideas, and serving as a committee member. I would like to extend thanks to Dr. Stephen E. Ralph and Dr. Zhong Lin Wang for serving as members of my committee.

Special thanks are also extended to my fellow colleagues in the Georgia Tech MBE group and the Intelligent Semiconductor Manufacturing group: Gregory Triplett, Frances Williams, Kyeongkyun Lee, Changhyun Yi, Tong-Ho Kim, and Georgiana Dagnall; extremely talented individuals whom I had the unique opportunity to work with. I gratefully acknowledge the financial support from the Air Force Research Laboratory (contract F33615-98-C-5428), GEM Consortium, and funding provided by the Georgia Institute of Technology President's Fellowship.

This thesis is dedicated to my mother Shirley, sisters Kimberly and Brittani, brothers Craig and Eric, for the understanding, patience, and endless support they have provided throughout my graduate studies. Without their constant encouragement, this work would never have been completed.

"Each leaf of this manuscript is a reminder of the many blessings bestowed upon me from the Lord above."

-Terence D. Brown 


\section{TABLE OF CONTENTS}

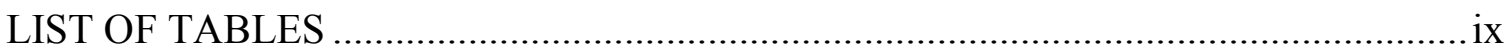

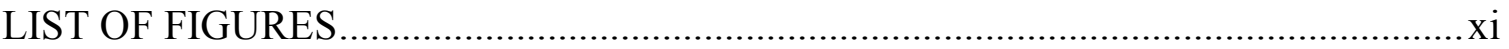

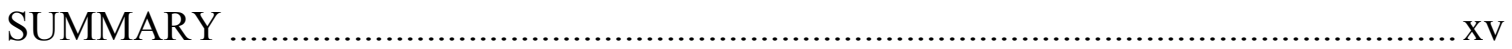

\section{CHAPTER}

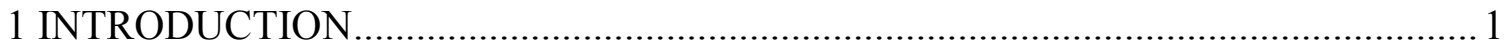

1.1 Molecular Beam Epitaxy ............................................................... 2

1.2 MBE Growth of III-V Compounds .......................................................... 3

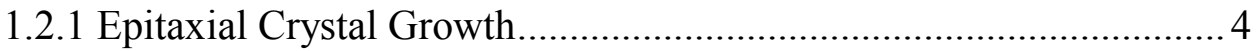

1.2.2 Chemical and Physical Processes................................................ 5

1.2.3 Adsorption and Surface Migration.............................................. 6

1.2.4 Lattice Matching and Strain ...................................................... 9

1.2.5 Heterostructure Growth..................................................... 12

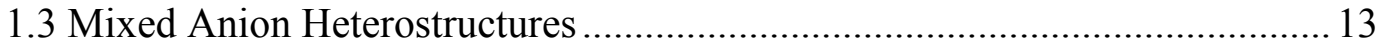

1.3.1 Anion Exchange and Device Performance................................. 13

1.3.2 Anion Exchange and Interface Control .................................... 15

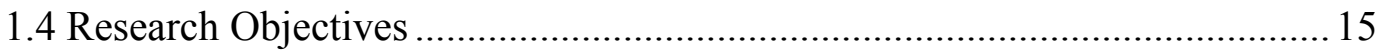

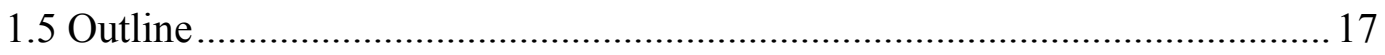

2 MICROSCOPIC PROCESSES AND MICROSTRUCTURES ................................... 18

2.1 Growth Kinetics and Thermodynamics................................................. 18

2.1.1 MBE Growth Models .................................................................. 19 
2.1.2 Growth Modes and Surface Morphology.

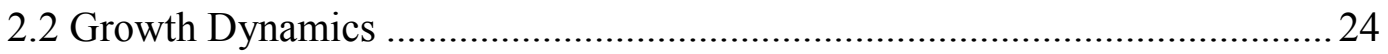

2.2.1 Interatomic Interactions at Surfaces ......................................... 24

2.2.2 Atomic Surface Models and Surface phases ................................26

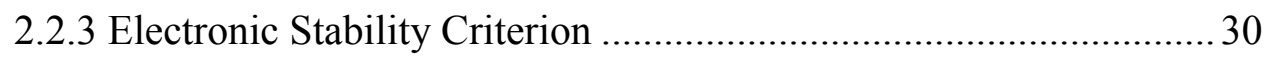

2.2.4 Atomic Growth Model and Growth Mechanisms ......................... 31

2.2.5 Atomic Surface Structures ......................................................... 34

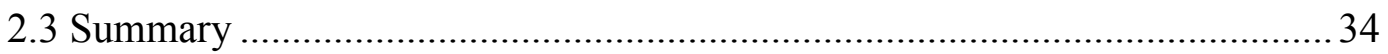

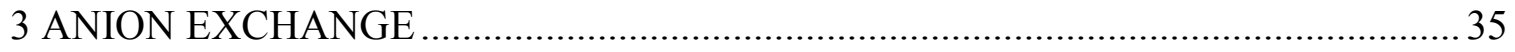

3.1 Anion Exchange \& Related Processes ...................................................... 35

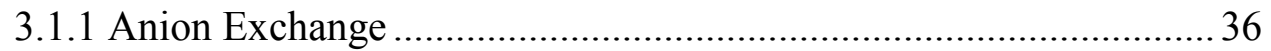

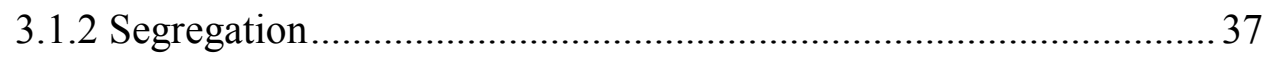

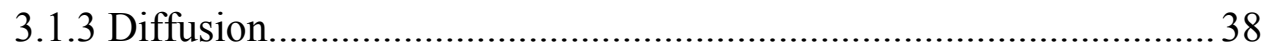

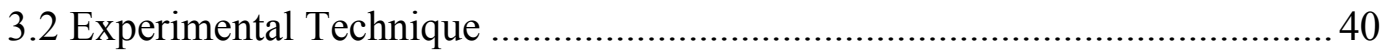

3.2.1 Design of Experiments for III-V Heterostructures....................... 40

3.2.2 Superlattice Structures for Analysis ........................................ 41

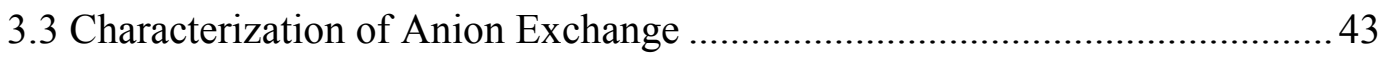

3.3.1 Reflection High-energy Electron Diffraction............................... 44

3.3.2 High-resolution X-ray Diffraction ......................................... 44

3.3.3 X-ray Photoelectron Spectroscopy …......................................... 45

3.3.4 High-resolution Transmission Electron Microscopy ...................... 45

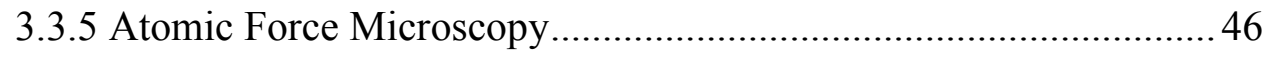


3.4 Semi-Empirical Modeling of Anion Exchange ………..................................4 46

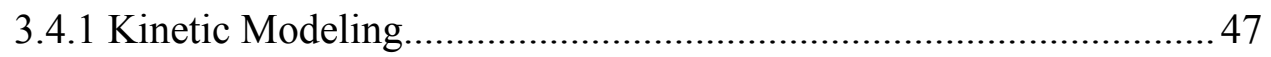

3.4.2 Neural Network Modeling …………………………………........53

3.4.3 Hybrid Neural Network Design ....................................................56

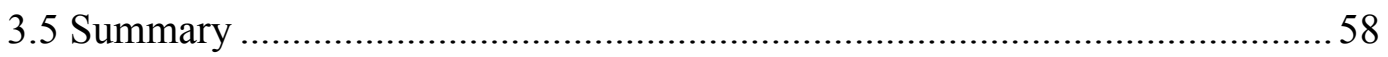

4 ANION EXCHANGE - INTERFACE PROFILES ................................................59

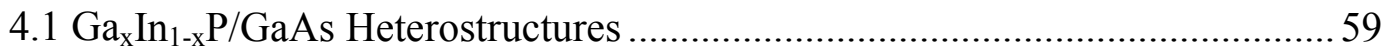

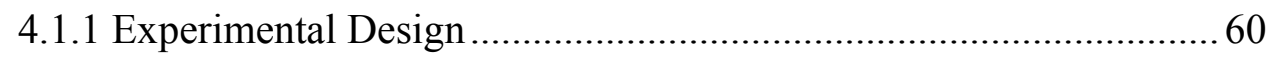

4.1.2 HRTEM and RHEED Analysis...................................................... 62

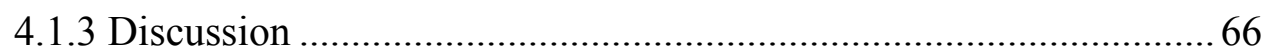

4.2 $\mathrm{GaAs}_{1-\mathrm{y}} \mathrm{Sb}_{\mathrm{y}} / \mathrm{GaAs}$ and $\mathrm{GaSb}_{1-\mathrm{y}} \mathrm{As}_{\mathrm{y}} / \mathrm{GaSb}$ Heterostructures .............................6 67

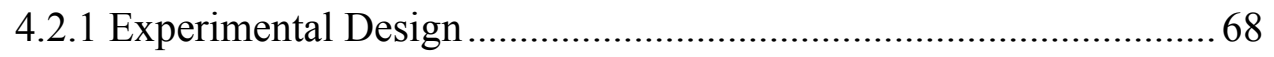

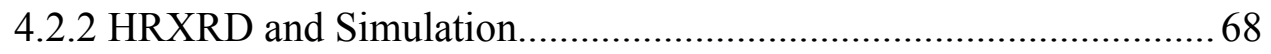

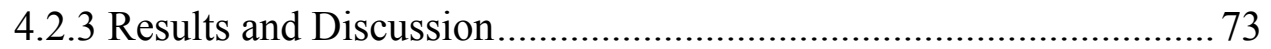

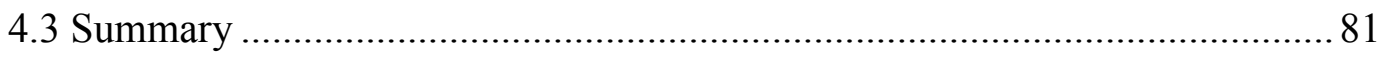

5 ANION EXCHANGE - ATOMIC SURFACE STRUCTURE ……………………......82

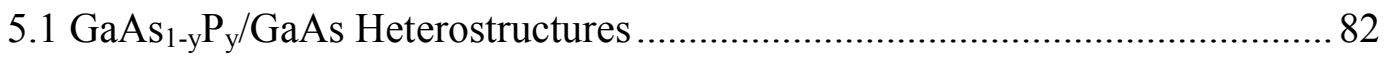

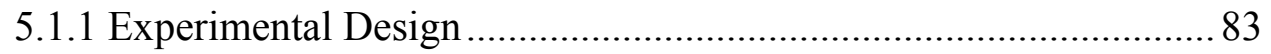

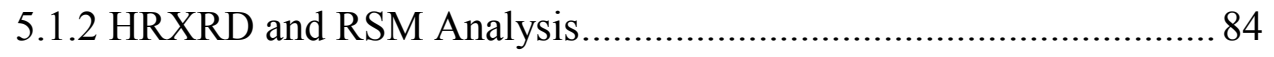

5.1.3 Neural Network Modeling ........................................................... 90

5.1.4 RHEED and Atomic Surface Structure ............................................. 94

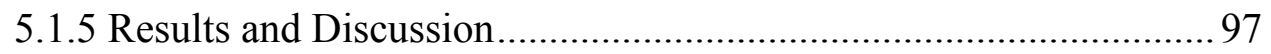

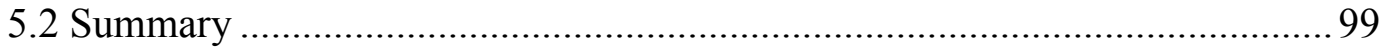


6 ANION EXCHANGE - HYBRID NEURAL NETWORK 100

6.1 Hybrid Neural Network Modeling..... 101

6.1.1 Experimental Design ....................................................... 102

6.1.2 Hybrid Neural Network Architecture........................................ 103

6.1.3 Kinetic Model Parameter Estimation ...................................... 110

6.1.4 Results and Discussion......................................................... 113

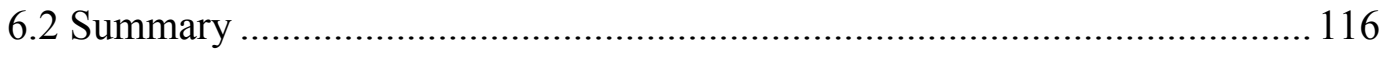

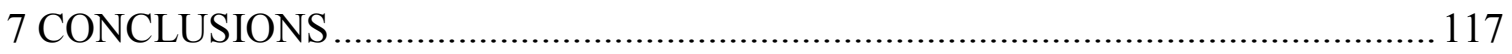

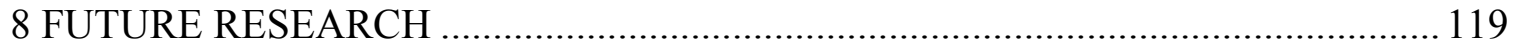

APPENDIX A - STM IMAGES AND ATOMIC SURFACE MODELS ..................... 120

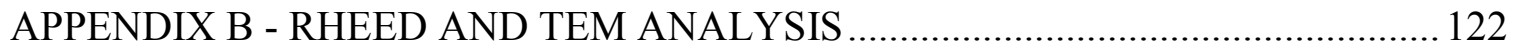

APPENDIX C - RHEED CHARACTERISTIC ABSENCES .................................... 125

APPENDIX D - OBJECT ORIENTED NEURAL NETWORK SIMULATOR............ 127

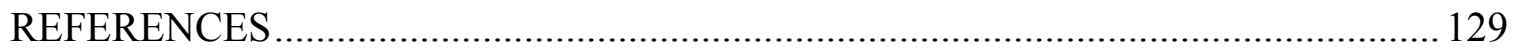

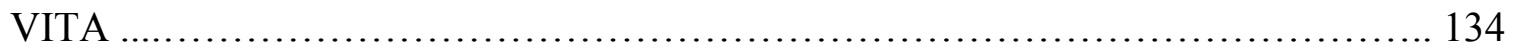




\section{LIST OF TABLES}

Table 4.1 The input parameter settings for the anion exchange As/P experimental design.

Table 4.2 The MBE growth conditions for the GaInP/GaAs SL structures.

Table 4.3 The MBE experimental conditions and HRXRD simulation data obtained for $\mathrm{Sb}_{2}$-exposed GaAs superlattices.

Table 4.4 The MBE experimental conditions and HRXRD simulation data obtained for $\mathrm{As}_{4}$-exposed GaSb superlattices.

Table 5.1 Input parameter settings for the anion exchange As/P experimental design.

Table 5.2 MBE growth conditions for the $\mathrm{P}_{2}$ exposure of As-stabilized GaAs.

Table 6.1 The physical definitions for each of the variables used in the kinetic model.

Table 6.2 Input parameter settings for the anion exchange As/P experimental design.

Table 6.3 The physical ranges of the kinetic parameters that are estimated by the hybrid neural network model.

Table 6.4 The hybrid neural network parameter estimations for $\mathrm{As}_{4}=4 \times 10^{-6}$ Torr.

Table 6.5 The hybrid neural network parameter estimations for $\mathrm{As}_{4}=2 \times 10^{-6}$ Torr.

Table 6.6 The kinetic parameters obtained by the semi-empirical hybrid neural network model for $\mathrm{As}_{4}=4 \times 10^{-6}$ Torr.

Table 6.7 The kinetic parameters obtained by the semi-empirical hybrid neural network model for $\mathrm{As}_{4}=2 \times 10^{-6}$ Torr.

Table B.1 Experimental Conditions, RHEED, and TEM data for the GaInP/GaAs SL structure (Sample 1). 
Table B.2 Experimental Conditions, RHEED, and TEM data for the GaInP/GaAs SL structure (Sample 2).

Table B.3 Experimental Conditions, RHEED, and TEM data for the GaInP/GaAs SL structure (Sample 3). 


\section{LIST OF FIGURES}

Figure 1.1 Schematic of a typical MBE growth chamber [3].

Figure 1.2 Schematic illustration of the surface processes occurring during film growth by MBE [8].

Figure 1.3 The surface interaction potential seen by a molecule impinging on the surface for chemisorbed and physisorbed states [8].

Figure 1.4 The structure for the diamond and zincblende lattice unit [12].

Figure 1.5 The relationship between band gap and lattice constant for semiconductor alloys in the InGaAsP and AlGaAsSb systems [12].

Figure 1.6 The structure of a five layer InAs/AlSb single quantum well heterostructure.

Figure 2.1 Model for the growth of GaAs from $\mathrm{Ga}$ and $\mathrm{As}_{2}$ [10].

Figure 2.2 Model for the growth of GaAs from $\mathrm{Ga}$ and $\mathrm{As}_{4}$ [11].

Figure 2.3 Schematic representation of the three MBE growth modes as a function of the surface coverage $\theta$ in monolayers.

Figure 2.4 The tetrahedral set of four $s p^{3}$ orbitals formed from one $s$ and three $p$ orbitals.

Figure 2.5 The $s p^{2}$ orbitals are formed from one $s$ and two $p$ orbitals. One $p$ orbital remains unchanged and is perpendicular to the plane of the hybrid orbitals.

Figure 2.6 The atomic structure model for the GaAs $(001)-(2 \times 4)$ surface with three As dimers in the surface layer of the unit cell. Filled circles denote As atoms and open circles denote Ga atoms [34].

Figure 2.7 The surface phase diagram and surface reconstruction models for the GaAs (001) surface. Filled circles denote As atoms and open circles denote $\mathrm{Ga}$ atoms.

Figure 2.8 The energy levels of dangling bonds for the GaAs (001) surface [42]. 
Figure 2.9 The sequence of electronically stable intermediate structures determined by the model for the complete monolayer growth cycle. The Ga atoms are indicated by $\circ$, and the As atoms are indicated by $\square$, with atoms closer to the surface indicated by larger symbols [43].

Figure 3.1 The atomic diffusion mechanisms in a 2-D lattice. For substitutional diffusion (left), the dissimilar anion moves among lattice vacancies. For interstitial diffusion (right), the dissimilar anion moves into the space between lattice sites.

Figure 3.2 The basic SL structure designed to study anion exchange using the $\mathrm{III}_{\mathrm{A}^{-}}$ $\mathrm{III}_{\mathrm{B}}-\mathrm{V}_{\mathrm{A}} / \mathrm{III}-\mathrm{V}_{\mathrm{B}}$ heterostructures grown in both the normal (white) and inverted (gray) interface configuration.

Figure 3.3 The SLS structure designed to study anion exchange using the III- $\mathrm{V}_{\mathrm{A}^{-}}$ $\mathrm{V}_{\mathrm{B}} / \mathrm{III}-\mathrm{V}_{\mathrm{A}}$ heterostructures. The structure consists of a mixed anion ternary coherently strained to the binary alloy surface.

Figure 3.4 The basic structure for the multilayer feed-forward neural network showing input, hidden, and output layers.

Figure 3.5 The structural design of the hybrid neural network model capable of predicting the composition at the interfaces of mixed anion III-V heterostructures.

Figure 4.1 The TEM image for Sample 1, the GaInP/GaAs SL structure composed of nine heterointerfaces.

Figure 4.2 The TEM image for Sample 2, the GaInP/GaAs SL structure composed of eleven heterointerfaces.

Figure 4.3 The TEM image for Sample 3, the GaInP/GaAs SL structure composed of thirteen heterointerfaces.

Figure 4.4 The initial HRXRD simulation structures representative of (a) $\mathrm{As}_{4}$ exposed GaSb superlattices and (b) $\mathrm{Sb}_{2}$-exposed GaAs superlattices.

Figure 4.5 The optimized simulation structures representative of (a) As 4 -exposed $\mathrm{GaSb}$ superlattices and (b) $\mathrm{Sb}_{2}$-exposed GaAs superlattices. The simulation structure and corresponding shutter sequence is provided for the first period of each superlattice.

Figure 4.6 The HRXRD $\boldsymbol{\theta} / 2 \boldsymbol{\theta}$ linescans (blue) and simulations (red) for superlattices formed by the $\mathrm{Sb}_{2}$ exposure of As-stabilized GaAs surfaces and immediate overgrowth of GaAs during MBE growth. 
Figure 4.7 The HRXRD $\boldsymbol{\theta} / 2 \boldsymbol{\theta}$ linescans (blue) and simulations (red) for superlattices formed by the $\mathrm{As}_{4}$ exposure of $\mathrm{Sb}$-stabilized $\mathrm{GaSb}$ surfaces and immediate overgrowth of GaSb during MBE growth.

Figure 4.8 The HRXRD $\boldsymbol{\theta} / 2 \boldsymbol{\theta}$ scan for a superlattice formed by the $\mathrm{As}_{2}$ exposure of $\mathrm{Sb}$-stabilized $\mathrm{GaSb}$ surfaces and immediate overgrowth of $\mathrm{GaSb}$ during MBE growth.

Figure 5.1 HRXRD $\boldsymbol{\theta} / 2 \boldsymbol{\theta}$ linescans for SLS structures formed by the $\mathrm{P}_{2}$-exposure of As-stabilized GaAs surfaces and immediate overgrowth of GaAs.

Figure 5.2 The effect of substrate temperature on the phosphorus composition at the interfaces of $\mathrm{GaAsP} / \mathrm{GaAs}$ heterostructures.

Figure 5.3 HRXRD $\boldsymbol{\theta} / 2 \boldsymbol{\theta}$ linescans for SLS structures formed by the $\mathrm{P}_{2}$-exposure of As-stabilized GaAs surfaces and immediate overgrowth of GaAs.

Figure 5.4 Reciprocal space maps around the (115) reflections obtained for $\mathrm{GaAsP} / \mathrm{GaAs}$ heterostructures.

Figure 5.5 The elastic strain in the GaAsP/GaAs SLS structures estimated using the average lattice mismatch.

Figure 5.6 Three-dimensional contour plot indicating the dependence of phosphorus composition at the GaAsP/GaAs interface on substrate temperature and phosphorus exposure time.

Figure 5.7 Three-dimensional contour plot indicating the dependence of phosphorus composition at the $\mathrm{GaAsP} / \mathrm{GaAs}$ interface on substrate temperature and phosphorus exposure time.

Figure 5.8 Sensitivity of the phosphorus incorporation mechanism with respect to substrate temperature for the high and low As-stabilizing pressures.

Figure 5.9 RHEED fractional order characterization technique allows $i n$-situ determination of surface stoichiometry and atomic surface structure [39].

Figure 5.10 Surface reconstruction map for the As-stabilized GaAs (001) surface. 96

Figure 5.11 Three-dimensional contour plot indicating the dependence of phosphorus composition at the $\mathrm{GaAsP} / \mathrm{GaAs}$ interface on the atomic surface structure of the group- $\mathrm{V}$ stabilized surface. 
Figure 5.12 Three-dimensional contour plot indicating the dependence of phosphorus composition at the $\mathrm{GaAsP} / \mathrm{GaAs}$ interface on the atomic surface structure of the group-V stabilized surface.

Figure 6.1 The structure for the multilayer feed-forward neural network showing input, hidden, and output layers.

Figure 6.2 The structural implementation of the hybrid neural network capable of estimating the kinetic parameters for the As/P heterostructures.

Figure 6.3 Experimental phosphorus composition versus the neural network predictions for $\mathrm{As}_{4}=4 \times 10^{-6}$ Torr.

Figure 6.4 Experimental phosphorus composition versus the neural network predictions for $\mathrm{As}_{4}=2 \times 10^{-6}$ Torr.

Figure C.1 RHEED data obtained during the $\mathrm{P}_{2}$ exposure of GaAs (001) surfaces. 125

Figure C.2 Determination of atomic surface structure using the RHEED fractional order characterization technique.

Figure D.1 The main control panel for ObOrNNS version 5.

Figure D.2 The network training interface for ObOrNNS version 5.

Figure D.3 The network testing interface for ObOrNNS version 5. 


\section{SUMMARY}

This research effort utilizes the statistical experimental design approach to investigate the anion exchange process during molecular beam epitaxy (MBE) growth of mixed anion III-V heterostructures. The research performed for this dissertation was part of a larger initiative to advance the understanding of the anion exchange process. The goal of the anion exchange project was to characterize at the atomic scale the mechanisms occurring at the interfaces of mixed anion heterostructures grown by MBE and correlate the effects of anion exchange with growth conditions, electronic properties, and device performance.

The objective of this dissertation research was to investigate the microscopic processes occurring at the interfaces of mixed anion III-V heterostructures grown by MBE. In particular, efforts were made to characterize the dominant chemical and physical mechanisms that lead to anion exchange and to develop semi-empirical models capable of predicting the atomic scale structure and composition at the interfaces of mixed anion heterostructures for the $\mathrm{As} / \mathrm{P}$ and $\mathrm{Sb} / \mathrm{As}$ material systems.

This research considers the MBE growth of 20-period superlattices (SLs) formed by allowing a dissimilar anion flux to impinge on a static group-V stabilized surface. Statistical experimental design was used to determine the effects of substrate temperature, V/III growth flux ratio, and anion exposure time on the anion exchange process. The superlattice structures were analyzed via high resolution x-ray diffraction (HRXRD) and simulation, RHEED analysis, x-ray photoelectron spectroscopy (XPS), and other techniques, which allowed for the determination of both chemical composition and 
atomic structure at the interfaces. Finally, a semi-empirical hybrid neural network was developed that quantifies the effects of MBE growth processes at the interfaces of mixed anion III-V heterostructures by incorporating a first principles kinetic model with backpropagation neural networks. These results were used in conjunction with HRXRD, RHEED, and XPS characterization data to investigate surface dynamics. 


\section{CHAPTER 1}

\section{INTRODUCTION}

Semiconductor heterostructures are the foundation for many current and future device technologies, such as terahertz microwave devices and quantum cascade lasers. The first GaAs/AlGaAs heterostructures were produced early in the development of molecular beam epitaxy (MBE) [1]. The investigation of heterostructure growth and the epitaxial growth of superlattices by MBE in the 1970s opened a whole new dimension for the study of quantum mechanical effects [2]. Since that time, the MBE growth technique has been used extensively in the development of new device concepts. Advanced devices, such as high electron mobility transistors (HEMTs), are currently being manufactured by MBE. The MBE growth technique has become a prominent highvolume production technology, and research into semiconductor heterostructure devices has gained considerable momentum. This is primarily due to the fact that the electronic and photonic industries are constantly expressing the need for higher frequency devices, and the demand for materials processing are increasingly stringent.

Mixed anion heterostructures have received considerable attention for applications in high speed electronic devices, infrared detectors, and quantum lasers. There are several researchers focusing on mixed anion materials in the development of nanostructures, considered the ultimate limit of device performance, where electrons and holes are confined in low dimensional structures. The MBE growth technique will play a critical role in the development of such devices because it is able to prepare compositionally abrupt interfaces with atomic layer precision. The study of microscopic 
processes occurring during epitaxial growth by MBE will allow the development of methods for fabricating low dimensional devices that operate using quantum effects.

This research focuses on the investigation of the microscopic processes occurring at the interfaces of mixed anion III-V heterostructures grown by MBE. The inherent features of the MBE growth technique make it well suited to research encompassing the study of growth processes, atomic surface structures, and materials properties.

\subsection{Molecular Beam Epitaxy}

$\mathrm{MBE}$ is a versatile thin-film growth technique used in the development of advanced compound semiconductor devices. Initially developed in the mid 1960s, MBE is characterized by a low growth rate $(0.5-1.0 \mu \mathrm{m} / \mathrm{h})$, low growth temperature $\left(\sim 580^{\circ} \mathrm{C}\right.$ for GaAs), and the ability to switch constituent beams in a fraction of the time required to grow a single atomic layer of material. The primary advantage of MBE is its ability to produce high-quality material with precise control over chemical composition, thickness, and doping concentrations [3]. Epitaxial growth in MBE proceeds by the impingement of molecular beams on a heated substrate in an ultra-high vacuum environment. The configuration of most MBE systems allows for the in-situ analysis of growth processes via reflection high-energy electron diffraction (RHEED). Figure 1.1 shows a diagram of a typical MBE growth chamber. Most commercially available MBE systems consist of three ultra-high vacuum chambers: a load-lock preparation chamber, an intermediate transfer/analytic chamber, and a growth/deposition chamber. Each of these chambers is independently pumped, and transfer of the sample between chambers is accomplished using magnetically coupled transfer rods, allowing the sample to be moved without breaking vacuum states. 


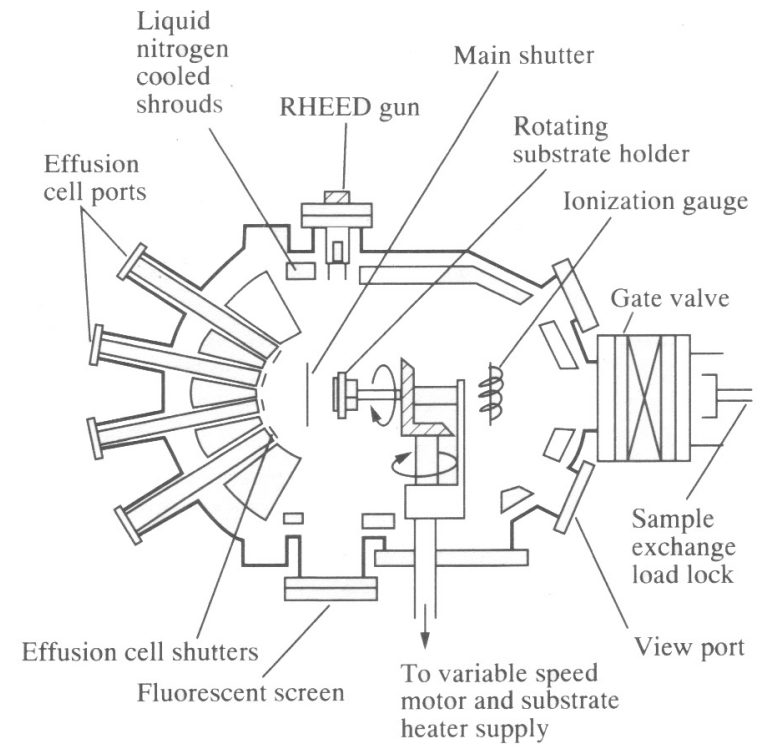

Figure 1.1 Schematic of a typical MBE growth chamber [3].

The unique potential of MBE for device applications has been confirmed by numerous achievements in the field of optoelectronic and high-speed electronic devices $[4,5]$. The capability of creating a wide variety of complex compositional profiles in semiconductors is an essential feature of the MBE growth technique. The following sections introduce the fundamentals of epitaxial crystal growth of semiconductors by MBE and explore the advantages of mixed anion heterostructures for device technology.

\subsection{MBE Growth of III-V Compounds}

Epitaxial growth is a subject with considerable practical application to the production of electronic, optoelectronic, and magnetic devices. This section begins with an introduction to the epitaxial crystal growth of semiconductors using the MBE growth technique. A review of the processes contributing to epitaxial growth is given, and the advantages of heteroepitaxial growth of semiconductors are explored. 


\subsubsection{Epitaxial Crystal Growth}

Thin-film epitaxial crystal growth occurs when the structure and orientation of the growing semiconductor are determined by the substrate. MBE is well suited for epitaxial growth of semiconductors, and the versatility of this growth method makes it very attractive for many applications. The fundamental processes for MBE growth of GaAs have been studied extensively, and studies of other binary compounds have indicated that similar behavior is observed [6,7]. MBE is a precisely controlled ultra-high vacuum technique, in which a heated substrate serves as a seed crystal, while molecular or atomic beams of the constituents impinge on its surface to form the desired thin film. The temperature of the substrate provides the kinetic energy to the impinging atoms and molecules for both dissociation and surface migration to suitable growth sites. In the MBE growth of III-V materials, all incident group-III atoms are assumed to be incorporated, while the group-V elements do not stick to the surface in the absence of a group-III flux. Therefore, the group-III flux determines the growth rate of the film and the excess group-V atoms are desorbed from the surface. The group-III elements and most dopants arrive at the surface in the form of atoms. In contrast, the group-V elements may arrive at the surface in the form of various molecular species. Most often, these species consist of tetrameric or dimeric molecules, depending on whether a standard effusion cell or a two-zone cracking cell is used during growth.

RHEED studies of MBE growth have provided significant insight into the mechanisms controlling the growth process. RHEED patterns reveal real-time surface dynamics, thereby allowing the determination of the evolving surface structure. RHEED has thus emerged as a powerful technique for both in-situ monitoring and atomic 
structure analysis. The RHEED characterization technique will be used extensively during the course of this research. The determination of surface reconstructions, growth rates, and atomic surface structure phases will be accomplished utilizing RHEED analysis. The characterization techniques used during the course of this research are discussed in Section 3.3.

\subsubsection{Chemical and Physical Processes}

The structural perfection of surfaces and interfaces in the epitaxial growth of semiconductors is intimately connected with the processes occurring on the surface. Both chemical and physical surface processes are of fundamental importance in understanding the mechanisms of epitaxial growth. MBE is generally regarded as a kinetically controlled growth method because of the low growth rate and low surface temperature under which growth takes place. A number of surface processes are involved in MBE growth. A schematic illustration of the processes occurring during film growth is provided in Figure 1.2. The most important processes for stoichiometric growth are adsorption of the impinging constituent atoms or molecules, surface migration and dissociation of adsorbed molecules, incorporation of constituent atoms at step edges, and thermal desorption of excess species [8].

Since these growth processes require the formation and breaking of chemical bonds, their rates are controlled by the kinetic energy provided by the substrate temperature. If the kinetic energy is insufficient for the completion of a step within a characteristic time interval, then the reaction will be unable to reach completion and the step is said to be kinetically limited. The surface processes occurring during MBE growth are characterized by a set of kinetic parameters that describe them quantitatively. 
Experimental determination of these thermally activated reaction rates can yield activation energies representative of the binding energies for the surface growth species. The emphasis on MBE growth kinetics stems from this fact, which provides a measurable link between kinetic parameters and complex atomic surface processes.

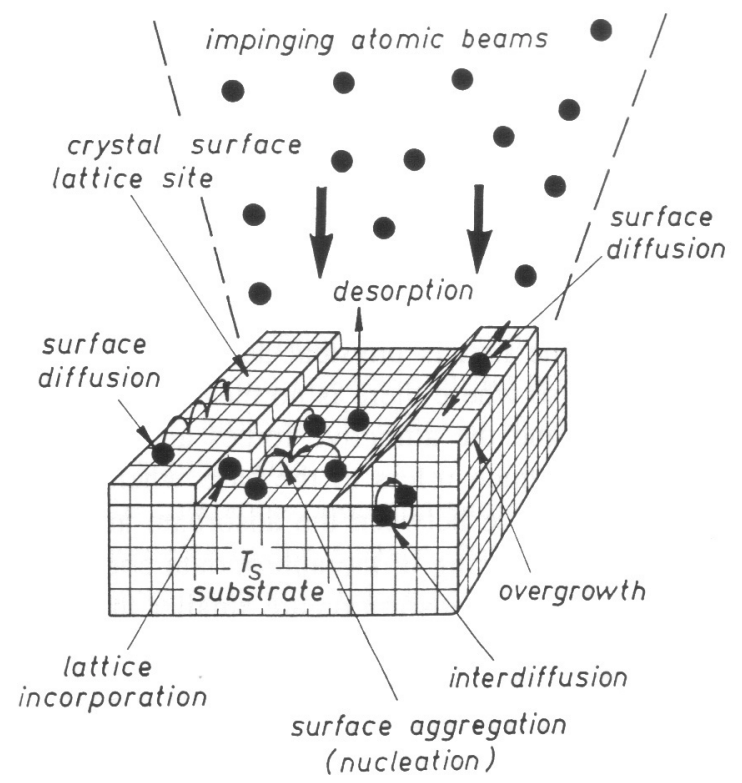

Figure 1.2 Schematic illustration of the surface processes occurring during film growth by MBE [8].

\subsubsection{Adsorption and Surface Migration}

In the study of epitaxial growth by MBE, it is conventional to distinguish between two types of adsorption. The first is physical adsorption, also called physisorption, which is the case where there is no electron transfer between the adsorbate (atom or molecule) and the surface, and the van der Waals forces bind the particle to the surface. This adsorption state is accompanied by fairly intensive surface migration of the adsorbate. The second type of adsorption is chemisorption, which refers to the case when a chemical reaction takes place between the adsorbate and the surface, leading to the formation of 
chemical bonds. The effects of physisorption and chemisorption are additive, i.e., the van der Waals forces are still present during chemisorption and contribute to the total binding energy of the adsorbate on the surface [9].

Due to the nature of the binding forces in physisorption, the adsorbate determines the interaction with the surface, and no dependence on surface orientation exists. Conversely, in chemisorption, the surface plays the dominant role. Studies of the interaction of $\mathrm{As}_{2}$ and $\mathrm{As}_{4}$ molecules with GaAs (001) surfaces indicate that growth proceeds through a two-step condensation process in which the molecular species reach the chemisorbed state via a precursor physisorbed state $[10,11]$. This investigation, which is reviewed in Section 2.1, provides the basis for models of MBE growth kinetics. The surface interaction potentials for chemisorbed and physisorbed states are shown in Figure 1.3. This diagram indicates that for a molecule adsorbed in the physisorbed state, the potential energy required to become chemisorbed at the surface is less than that which is required for desorption from the surface, i.e. $E_{\mathrm{a}}<E_{\mathrm{dp}}$. It should be noted that a variety of surface potential configurations exist during MBE growth.

The quantitative description of adsorption is obtained by defining the thermal accommodation coefficient and the sticking coefficient. During MBE growth, the atoms that are impinging on the surface have an energy distribution corresponding to the effusion cell temperature $T_{\mathrm{i}}$. Upon arriving at the substrate surface, which has temperature $T_{\mathrm{s}}$, the atoms either reevaporate immediately, with an energy corresponding to temperature $T_{\mathrm{e}}$, or exchange energy with the atoms on the surface until they are in thermodynamic equilibrium at $T_{\mathrm{s}}$ [8]. The thermal accommodation coefficient $a$ is a 
measure of the extent to which atoms and molecules arriving at the surface reach thermal equilibrium with the substrate, and is expressed

$$
a=\frac{T_{i}-T_{e}}{T_{i}-T_{s}} .
$$

The sticking coefficient $s$ is defined as the ratio of the number of atoms adhering to the substrate surface to the number of atoms arriving at the surface, and is expressed

$$
s=\frac{N_{a d h}}{N_{t o t}} .
$$

It should be evident based on the definitions provided in this section that physisorption and chemisorption have different sticking coefficients. The sticking coefficient for chemisorption may be dependent on the substrate orientation, the species of impinging molecules, surface coverage by adsorbed atoms, and surface reconstruction. Conversely, the sticking coefficient for physisorption usually shows little or no dependence on the orientation and coverage of the substrate surface.

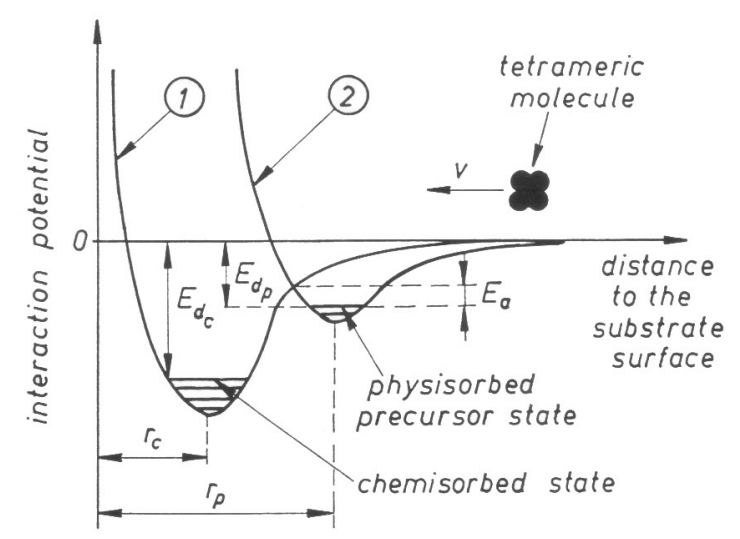

Figure 1.3 The surface interaction potential seen by a molecule impinging on the surface for chemisorbed and physisorbed states [8]. 


\subsubsection{Lattice Matching and Strain}

The growth of binary semiconductors on native substrates (e.g., GaAs-on-GaAs) results in a natural matching of the crystal lattice and formation of high-quality single crystal layers. The inherent constraints of epitaxial crystal growth require that the growing crystal layers be lattice-matched to the substrate. In other words, epitaxial growth of a crystal layer can be accomplished if the lattice structure and lattice constant for the growing material are the same as that of the substrate. This is the criterion for lattice matching. Most semiconductors must be lattice matched to within $0.1 \%$ to prevent the introduction of strain into the epitaxial layer.

The III-V semiconductors in the InGaAsP and $\mathrm{AlGaAsSb}$ material systems each have a zincblende structure. This structure, a variant of the diamond lattice, is shown in Figure 1.4 and consists of two inter-penetrating $f c c$ sublattices with group-III atoms on one sublattice and group- $\mathrm{V}$ atoms on the other. As a result, epitaxial layers of various ternary alloys in these material systems can be grown on GaAs, InP, or GaSb substrates with little or no lattice mismatch. The relationship between the energy band gap $\mathrm{E}_{\mathrm{g}}$ and the lattice constant for alloys in these material systems is illustrated in Figure 1.5. For example, as the ternary compound InGaAs is varied over its compositional range, the band gap changes from 0.36 to $1.43 \mathrm{eV}$, and the lattice constant of the crystal varies from $6.0584 \AA$ for InAs to $5.6533 \AA$ for GaAs [12]. The $\operatorname{In}_{x} G a_{1-x} A s$ alloy can be grown lattice matched to an InP substrate using a ternary composition $x=0.53$. Similarly, the ternary $\mathrm{Ga}_{\mathrm{x}} \mathrm{In}_{1-\mathrm{x}} \mathrm{P}$ with composition $x=0.51$ can be grown lattice matched to a GaAs substrate. There are several options for extending the range of available band gaps using alloy compositions grown lattice matched on specific substrates. The variation of ternary 
composition on the group- $\mathrm{V}$ sublattice allows for ternary alloys such as $\mathrm{GaAs}_{0.51} \mathrm{Sb}_{0.49}$ to be lattice matched to an $\mathrm{InP}$ substrate. Quaternary alloys such as $\mathrm{In}_{\mathrm{x}} \mathrm{Ga}_{1-\mathrm{x}} \mathrm{As}_{\mathrm{y}} \mathrm{P}_{1-\mathrm{y}}$ can also provide additional flexibility in choosing a particular band gap by allowing for variation of compositions on both the group-III and group-V sublattices while being lattice matched to binary substrates such as GaAs or InP.

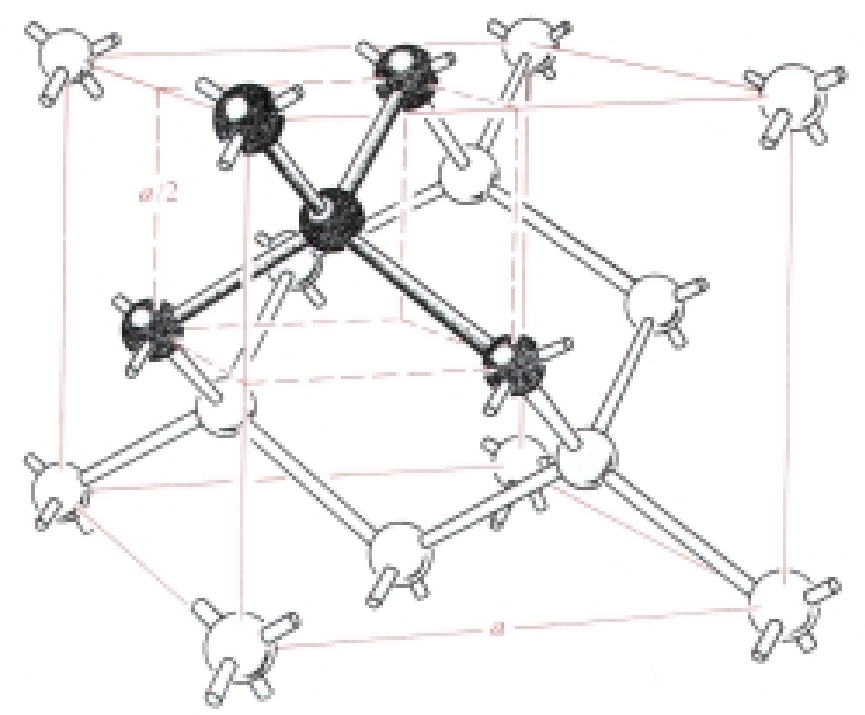

Figure 1.4 The structure for the diamond and zincblende lattice unit [12].

The MBE growth technique is not limited to lattice-matched epitaxial layers. Alloys can be grown with lattice constants differing from those of the underlying substrate if the thickness of the layer is less than a critical thickness. These thin layers of lattice-mismatched crystals will be strained to match the substrate, and a coherent epitaxial layer will be formed. Specifically, for cubic materials, the layer undergoes tetragonal distortion during growth, resulting in layer compression or tension along the surface plane. This type of layer formation is defined as pseudomorphic, as the semiconductor layer is not lattice matched to the substrate without strain. As the 
thickness of the alloy increases, the strain energy in the layer builds until a "critical thickness" is reached when it is energetically favorable for defects, such as dislocations, to be introduced. These dislocations relieve strain, but also degrade the structural, electrical, and optical quality of epitaxial layers, often making them unsuitable for device applications.

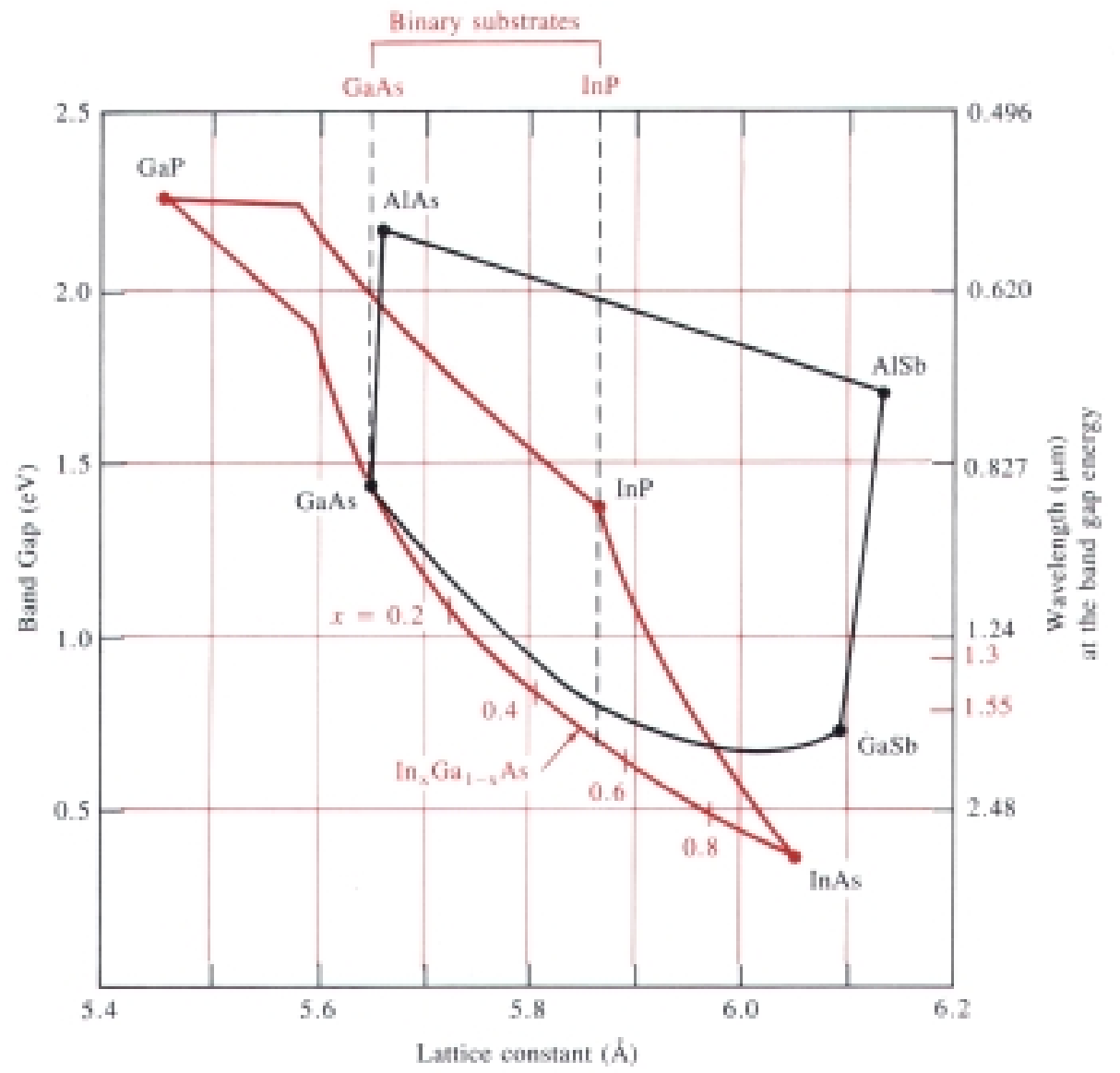

Figure 1.5 The relationship between band gap and lattice constant for semiconductor alloys in the InGaAsP and AlGaAsSb systems [12]. 


\subsubsection{Heterostructure Growth}

Semiconductor heterostructure formation is the process of growing two dissimilar semiconductors epitaxially in a series of layers. The study of heteroepitaxial material systems has long been a primary focus of MBE research. The electronic properties of many compound semiconductor devices are highly dependent on heterointerface structure and composition. Early studies in the field of heterostructure growth focused on the GaAs-AlAs system. These heterostructures were used to design quantum wells and superlattices that immediately rose to the forefront in investigations of electronic properties, carrier transport and growth dynamics $[13,14]$. The advantage of the GaAsAlAs system stems from the fact that both AlAs and GaAs have lattice constants close to $5.65 \AA$. Therefore, the ternary alloy $\mathrm{Al}_{\mathrm{x}} \mathrm{Ga}_{1-\mathrm{x}} \mathrm{As}$ has essentially the same lattice constant over the entire range of compositions $x$ from AlAs to GaAs. This allows for band gap selection using the composition of the ternary alloy AlGaAs, which can be grown on GaAs substrates with very little lattice mismatch. The GaAs-AlAs system has remained a vital tool for research in many areas of solid state physics.

The current trend in heterointerface design involves the growth of mixed anion materials, i.e., ternary alloys that contain two group-V elements. Heterostructures composed of these materials contain dissimilar anions across the interface (e.g., $\mathrm{GaAs}_{0.51} \mathrm{Sb}_{0.49}$-InP and $\mathrm{AlSb}_{0.29} \mathrm{P}_{0.71}$-GaAs). These materials offer significant advantages in band gap engineering. For instance, the band gap of GaAsP is suitable for both visible and IR emitters and detectors for the 0.7-2.0 $\mu \mathrm{m}$ range, and InAsP based structures are useful for devices in the 1.3-3.0 $\mu \mathrm{m}$ range. In heteroepitaxy, there are several important factors controlling the growth process. These include, but are not limited to, strain or 
misfit at the interface, the density of coincident atomic sites at the interface, and interatomic interactions of atoms at the interface. For mixed anion materials, these factors can be adversely affected by physical processes such as anion exchange. Anion exchange results from the tendency for the group $\mathrm{V}$ elements in mixed anion heterostructures to substitute across the interface. This leads to non-abrupt interfaces and modification of the electronic properties of these materials.

\subsection{Mixed Anion Heterostructures}

Mixed anion heterostructures grown by MBE have become the focus of interest for designing next-generation devices such as long-wavelength lasers, high-frequency HEMTs, and heterojunction bipolar transistors (HBTs). This section reviews the effects of anion exchange on the properties of current mixed anion device technologies and introduces the relevant materials issues related to anion exchange at the interfaces of mixed anion heterostructures.

\subsubsection{Anion Exchange and Device Performance}

In order to fully understand the effects of anion exchange in mixed anion device technologies, consider the MBE growth of a five layer InAs/AlSb single quantum well heterostructure. The quantum well, illustrated in Figure 1.6, is of great interest for the design of low-voltage, high-frequency HEMTs. The primary advantages of this material system include the high electron mobility $\left(30,000 \mathrm{~cm}^{2} / \mathrm{V} \cdot \mathrm{s}\right)$ and velocity $\left(4 \times 10^{7} \mathrm{~cm} / \mathrm{s}\right)$ of InAs [15], and the large conduction band offset between InAs and AlSb (1.35 eV) [16]. This large conduction band offset results in good carrier confinement in the InAs quantum well. During growth of these heterostructures there are two critical interfaces at 
which anion exchange may occur, above and below the InAs channel layer. In the event that anion exchange occurs, interface roughness and the formation of dislocations due to anion exchange can lead to carrier scattering and reduced mobilities [17]. Similar effects have been reported in quantum well lasers and modulation doped quantum well structures, where anion exchange can lead to a decrease in carrier transport properties and degradation of device performance.

\begin{tabular}{|c|c|c|}
\hline \multirow{5}{*}{$\begin{array}{l}\text { Critical - } \\
\text { Inte rfaces }\end{array}$} & $\mathrm{AlSb}$ & $10 \mathrm{~nm}$ \\
\hline & InAs channel & $15 \mathrm{~nm}$ \\
\hline & $\mathrm{AlSb}$ & $2 \mu \mathrm{m}$ \\
\hline & GaAs buffer & $250 \mathrm{~nm}$ \\
\hline & \multicolumn{2}{|c|}{ GaAs (001) substrate } \\
\hline
\end{tabular}

Figure 1.6 The structure of a five layer InAs/AlSb single quantum well heterostructure.

While anion exchange can result in numerous device issues, such as band gap discontinuities and low carrier efficiency, applications that exploit the anion exchange process have also emerged. The potential for anion exchange to be used for modification of nanostructures has been demonstrated by Shen et al. [18]. Applications such as these will greatly benefit from quantitative explanations of the microscopic processes that contribute to anion exchange. The extent to which reaction mechanisms occur, as well as kinetic limitations, must be investigated before control of anion intermixing can be fully realized. A better understanding of anion exchange and related processes occurring during the growth of mixed anion heterostructures is critical in the development of mixed anion device technologies. 


\subsubsection{Anion Exchange and Interface Control}

Several studies have investigated interfacial control in mixed anion heterostructures, and many have indicated that the exchange reaction is difficult to suppress [19]. Advanced growth techniques that have shown some success in interface control of anion intermixing include migration-enhanced epitaxy (MEE), V/III pressure ratio minimization, growth interrupt schemes, and single monolayer group-III deposition. A common approach for analysis of anion exchange in the $\mathrm{As} / \mathrm{Sb}$ material system has been the characterization of $\operatorname{InAs} /(\mathrm{Ga}, \mathrm{Al}) \mathrm{Sb}$ superlattices, and $\mathrm{InGaSb} / \mathrm{InAs}$ quantum well structures using various in-situ and ex-situ techniques [20,21]. Work performed by Kaspi et al. [22] used desorption mass spectrometry (DMS) data in conjunction with $\mathrm{XRD}$ analysis to optimize the $\mathrm{As}_{2}$ exposure time, resulting in atomically abrupt InAs/GaSb interfaces. Investigations performed by Xie et al. [23] determined that surfaces terminated by one monolayer of $\mathrm{Ga}$ or In produce a critical $\mathrm{As}_{2}$ flux below which As-for-Sb exchange is drastically reduced. While significant results have been obtained in minimizing anion intermixing, interface control has been addressed primarily in this respect.

\subsection{Research Objectives}

The fundamental principles governing the MBE growth of mixed anion III-V materials are thoroughly investigated in this dissertation. This research considers the MBE growth of 20-period superlattices formed by allowing a dissimilar anion flux to impinge on a static group-V stabilized surface. Statistical experimental design was used to determine the effects of substrate temperature, V/III growth flux ratio, and anion exposure time on the anion exchange process. This research encompasses three essential 
interrelated modules: (1) MBE growth and characterization; (2) kinetic and neural network modeling; and (3) semi-empirical modeling. Each of these modules provides detailed information about the microscopic processes occurring at the interfaces of mixed anion III-V heterostructures.

The primary objective of MBE growth and characterization was to determine the structural properties of the MBE grown superlattices, designed to study anion intermixing. Specifically, characterization techniques were performed in an effort to determine the strain and composition profile at the mixed anion interfaces for each material system. As discussed in Section 3.3, several in-situ and ex-situ characterization techniques have been employed for this research effort. The principal technique used for the analysis of anion intermixing for the SL structures was ex-situ HRXRD. In addition, the surface reconstructions for all of the structures grown were observed in-situ by RHEED, and several of the SL structures were grown with no cap layer, allowing chemical analysis by XPS and analysis of surface roughness by atomic force microscopy (AFM) to be performed on the final interface.

The objective of kinetic and neural network modeling was to develop a kinetic model capable of predicting dissimilar anion composition and implement backpropagation neural networks to obtain preliminary results for anion intermixing at the interfaces of mixed anion III-V heterostructures. The details of the kinetic model developed for this research are discussed in Section 3.4. The neural network modeling technique was performed for the $\mathrm{GaAs}_{1-\mathrm{y}} \mathrm{P}_{\mathrm{y}} / \mathrm{GaAs} \mathrm{SL}$ structures, and results for dissimilar anion composition at the interface were achieved. 
The objective of semi-empirical modeling was to develop a hybrid neural network capable of quantifying the effects of MBE growth processes at the interfaces of mixed anion III-V heterostructures. The hybrid neural network designed for this research was implemented using a software application built based on ObOrNNS, the object-oriented neural network simulator developed by the Intelligent Semiconductor Manufacturing group at Georgia Tech. The ObOrNNS software package has been redesigned with a completely new architecture and programmed using Java. ObOrNNS implements semiempirical modeling by allowing physical models to be incorporated with a neural network via a Java plug-in interface.

\subsection{Outline}

This dissertation is structured as follows. Chapter 2 contains an introduction to the growth kinetics and growth dynamics of MBE. The theoretical and practical aspects of the anion exchange process are discussed in Chapter 3, along with the detailed experimental procedures and development of the kinetic model. In Chapter 4 techniques for determining the interface profiles of heterostructures are introduced. Chapter 5 describes the neural network modeling of anion exchange and the dependence of anion exchange on atomic surface structure. Chapter 6 provides the details concerning the design and development of the hybrid neural network. Finally, Chapter 7 provides the conclusions obtained during this research effort, and Chapter 8 contains suggestions for future work. 


\section{CHAPTER 2}

\section{MICROSCOPIC PROCESSES AND MICROSTRUCTURES}

The study of epitaxial crystal growth at surfaces is divided into two primary areas. Growth kinetics considers the variation in reaction rates as a function of external parameters such as substrate temperature, growth fluxes, and flux ratios. Growth dynamics is the study of the detailed mechanisms occurring at the atomic scale that characterize a given process [24]. This chapter introduces the present state of knowledge concerning the processes controlling crystal growth by MBE.

\subsection{Growth Kinetics and Thermodynamics}

The MBE growth process is, by definition, a dynamic phenomenon. Consideration of thermodynamics as well as kinetics is therefore essential. While thermodynamics represents the behavior of the physical system at equilibrium, kinetics controls the ability of the system to move toward the equilibrium state within the given conditions [25]. The term equilibrium as it relates to MBE implies that the adatom arrival rate (deposition rate for atoms and molecules reaching the substrate surface) is infinitely slow compared to the rates of kinetic processes. When equilibrium conditions for MBE growth are satisfied, complex incorporation kinetics on the surface can be determined using RHEED and various in-situ spectroscopic techniques. 


\subsubsection{MBE Growth Models}

During the early stages of MBE growth studies, experiments using modulated molecular beams, performed by Foxon et al. revealed detailed information about the kinetic factors controlling the growth process. Measurements of thermal accommodation coefficients, surface lifetimes, sticking coefficients, desorption energies, and reaction order were performed. The interaction of both $\mathrm{As}_{2}$ and $\mathrm{As}_{4}$ molecules with GaAs (001) surfaces was established using these results.

In the growth of $\mathrm{GaAs}$ from $\mathrm{Ga}$ and $\mathrm{As}_{2}$, the $\mathrm{As}_{2}$ molecule is initially adsorbed in a mobile precursor physisorbed state. During surface migration, if the $\mathrm{As}_{2}$ molecule encounters paired $\mathrm{Ga}$ lattice sites on the surface, dissociative chemisorption of the adsorbed molecule occurs [10]. The growth model for this process is illustrated in Figure 2.1. In the absence of available Ga lattice sites, the $\mathrm{As}_{2}$ molecule has a measurable surface lifetime, but no dissociation or permanent condensation occurs. The sticking coefficient of $\mathrm{As}_{2}$ is thus proportional to the gallium flux and approaches unity for a Garich surface. Excess $\mathrm{As}_{2}$ molecules are desorbed from the surface, leading to stoichiometric growth of GaAs.

GaAs grown from $\mathrm{Ga}$ and $\mathrm{As}_{4}$ involves a more complex process [11]. Figure 2.2 illustrates the growth model for this process. The $\mathrm{As}_{4}$ molecule is initially adsorbed in a mobile precursor state with a measurable surface lifetime. The growth process proceeds via a pairwise interaction of adsorbed $\mathrm{As}_{4}$ molecules on adjacent Ga lattice sites, leading to a process of dissociative chemisorption. The sticking coefficient of $\mathrm{As}_{4}$ molecules is a function of the Ga beam flux and is always less than or equal to 0.5. If the As4 surface population is small compared to the number of available Ga sites, then the requirement 
for pairwise interaction of $\mathrm{As}_{4}$ molecules is the rate-limiting step, and the desorption of $\mathrm{As}_{4}$ is a second-order kinetic process with respect to its adsorption. When the $\mathrm{As}_{4}$ surface population is increased, the probability that adsorbed $\mathrm{As}_{4}$ molecules arrive at adjacent lattice sites increases, and the desorption rate becomes proportional to the number of molecules being supplied (i.e., a first-order kinetic process). As a result, a larger flux ratio of $\mathrm{As}_{4}$ to gallium as compared to $\mathrm{As}_{2}$ to gallium is required to produce stoichiometric GaAs. When excess $\mathrm{As}_{4}$ molecules are supplied to the surface, the growth process is referred to as "arsenic-stabilized."

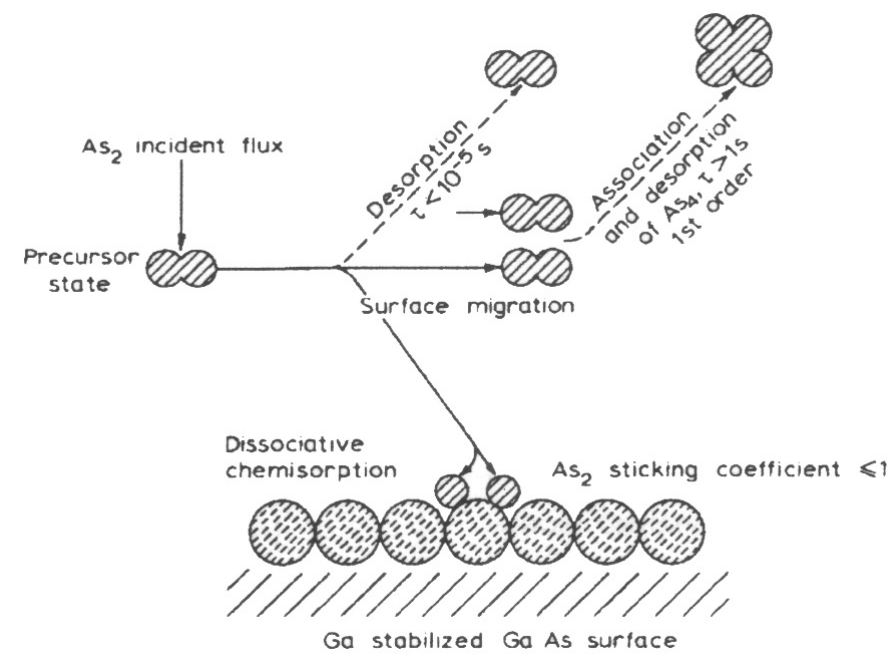

Figure 2.1 Model for the growth of $\mathrm{GaAs}$ from $\mathrm{Ga}$ and $\mathrm{As}_{2}[10]$.

It should be noted for both growth models that As desorption increases with increasing substrate temperature, a process that results in an increase in $\mathrm{Ga}$ chemical potential on the surface (i.e., a Ga adatom population), and ultimately a Ga-stabilized structure being established. In order to maintain an As-stabilized structure, the As lost as a result of an increase in temperature must be replaced by increasing the As beam flux such that the arsenic supply rate exceeds the rate of arsenic desorption. Therefore, the 
surface structure during growth is dependent on the V/III flux ratio, the substrate temperature, and the absolute magnitude of the fluxes. These growth models established from kinetic measurements are valid for a number of III-V compounds.

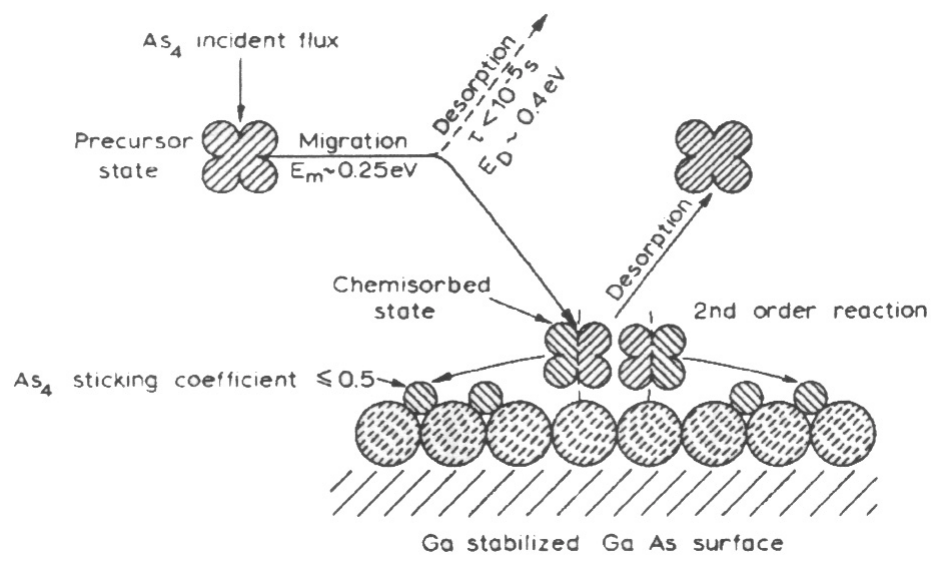

Figure 2.2 Model for the growth of $\mathrm{GaAs}$ from $\mathrm{Ga}$ and $\mathrm{As}_{4}[11]$.

\subsubsection{Growth Modes and Surface Morphology}

There are three equilibrium growth modes that are generally accepted as occurring in epitaxial crystal growth when interdiffusion is not permitted. Bauer was the first to classify these growth modes and their resulting surface morphologies in thermodynamic terms [26]. The growth modes, illustrated in Figure 2.3, are referred to as the 2-D layer-by-layer or Frank-van der Merwe (FM) growth mode, the 3-D island or Volmer-Weber (VW) growth mode, and the layer-plus-island or Stranski-Krastanov (SK) growth mode. The FM growth mode is often observed for homoepitaxial growth of semiconductors. This growth mode occurs because the deposited atoms are more strongly attracted to the substrate than they are to each other, leading to the formation of successive complete layers. Alternatively, the VW growth mode occurs when the attraction of the deposited atoms with each other is stronger than that of the deposited 
atoms with the substrate. In this case, islands form on the surface, a process typically observed in heteroepitaxial growth of materials with a large lattice-mismatch. The SK growth mode, the intermediate case, begins with the formation of a few monolayers, after which subsequent layer growth is unfavorable and islands are formed on the initial layers, often referred to as "wetting" layers.

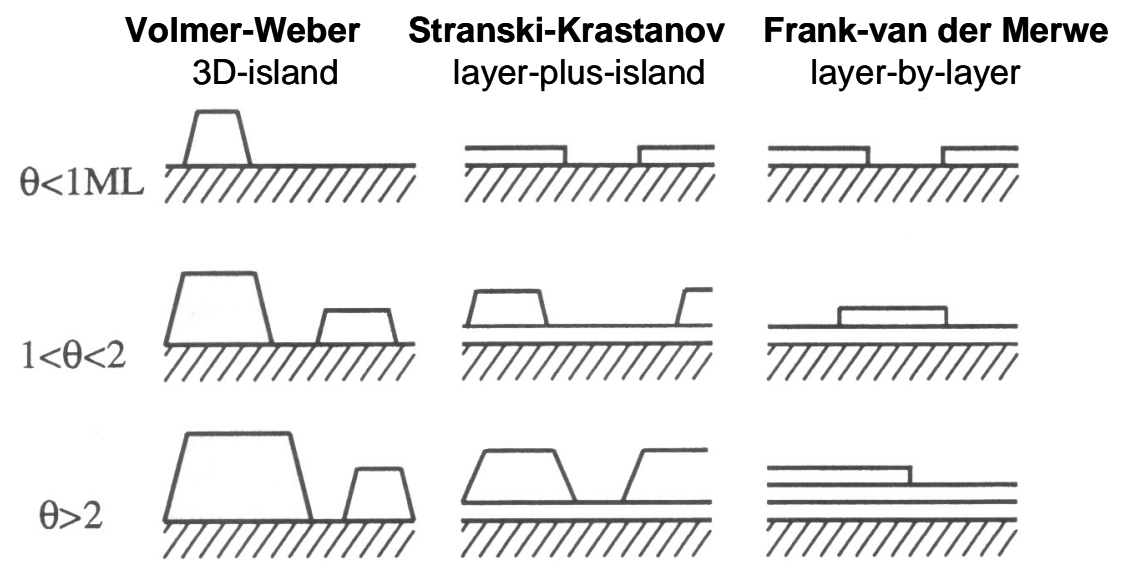

Figure 2.3 Schematic representation of the three MBE growth modes as a function of the surface coverage $\theta$ in monolayers.

These growth modes can be understood from thermodynamic arguments based on the interfacial free energies. Surface energies per unit area are denoted by $\gamma\left(\mathrm{eV} / \mathrm{nm}^{2}\right)$, where $\gamma_{i}, \gamma_{e}$, and $\gamma_{s}$ are the free energy of the interface, the epilayer, and the substrate, respectively. The FM growth mode is favored if the total free energy of the epilayer and the interface is less than that of the substrate, $\gamma_{i}+\gamma_{e}<\gamma_{s}$. In this case, during epilayer formation, the free energy decreases monotonically toward a steady-state value close to that for a bulk crystal of the layer material. If the free energy of the epilayer and the interface is greater than that of the substrate, $\gamma_{i}+\gamma_{e}>\gamma_{s}$, then the VW growth mode is favored. In this case, the free energy increases when epilayer formation begins and the 
layer becomes thermodynamically unstable, leading to a break-up of the layer into regions or islands.

The SK growth mode occurs because the free energy of the interface $\left(\gamma_{i}\right)$ increases as the layer thickness increases, resulting in strained layer growth characterized by pseudomorphic layer formation. While this 2-D layer formation is the initial stage of the SK growth mode, the build-up of strain energy eventually makes layer growth unfavorable. At this stage, continued growth results in the formation of 3-D islands that reduce strain through the formation of misfit dislocations. There is an alternative scenario for the SK growth mode that results in the formation of coherent, dislocationfree 3-D islands atop the wetting layers. These structures, called "quantum-dots," have been observed in the InAs on GaAs (001) system and several other semiconductor systems [27].

In general, these equilibrium growth modes are merely the limiting cases for actual material systems. Epitaxial crystal growth by MBE at temperatures below $600^{\circ} \mathrm{C}$ principally involves kinetic processes with rates that are comparable to or slower than the rate of deposition, i.e., non-equilibrium growth. Under these conditions, surface morphology may vary significantly from that predicted by the equilibrium growth modes. The experiments performed in this research effort were conducted using a "non-growing" (no incident group-III) anion exposure technique that approaches equilibrium and is bound by the SK growth mode, allowing for the analysis of anion exchange via kinetic and thermodynamic principles. Recent investigations by Wang et al. [28] have led to the development of a thermodynamic model that predicts the occurrence of anion exchange for growth by MBE. 


\subsection{Growth Dynamics}

The surface of a semiconductor undergoes a significant number of chemical reactions during growth. As the surface evolves, the bulk crystalline structure at the surface is not preserved. The surface atoms reconfigure themselves in both atomic position and bonding arrangement with respect to the bulk condition in order to lower the surface free energy. The surface, which is thus defined by a unit cell that has a different periodicity than the underlying bulk, is said to reconstruct. The surface reconstruction of a semiconducting material is the starting point for understanding the mechanisms of growth [29]. In general, reconstruction at semiconductor surfaces is not simple, and it is often difficult to understand the detailed geometry. This section introduces the principal reconstructions of the GaAs (001) surface and reviews the atomic growth model for this system.

\subsubsection{Interatomic Interactions at Surfaces}

The concept of bonding in solids is of great importance in growth dynamics, as this aspect is central in determining and understanding the subtle atomistic effects occurring at surfaces and interfaces [30]. A chemical bond can be simply viewed as a force that causes groups of atoms to behave as a unit. Bonds occur when groups of atoms are more stable (lower in energy) together than they are as separate atoms. The bonding of elemental solids can be classified as metallic, covalent, or ionic. In some cases, when these are not present, van der Waals bonding may occur. For the purposes of this research, we will focus on covalent bonding, where electrons are shared by atoms and each electron pair constitutes a covalent bond. 
In order to understand the role of covalent bonding in the formation of reconstructions at semiconductor surfaces, the arrangement of bonds for bulk semiconductors must be introduced. In the diamond structure solids, e.g., silicon $\left(4 s^{2} 4 p^{2}\right)$, the tetrahedral bonding comes from $s p^{3}$ hybridization. This hybridization is a mixing of the native atomic orbitals to form special orbitals for bonding. The $s p^{3}$ orbitals, shown in Figure 2.4, have a $109.5^{\circ}$ interbond angle and are formed from one $s$ and three $p$ orbitals. They occur whenever a set of equivalent tetrahedral atomic orbitals is required by an atom.

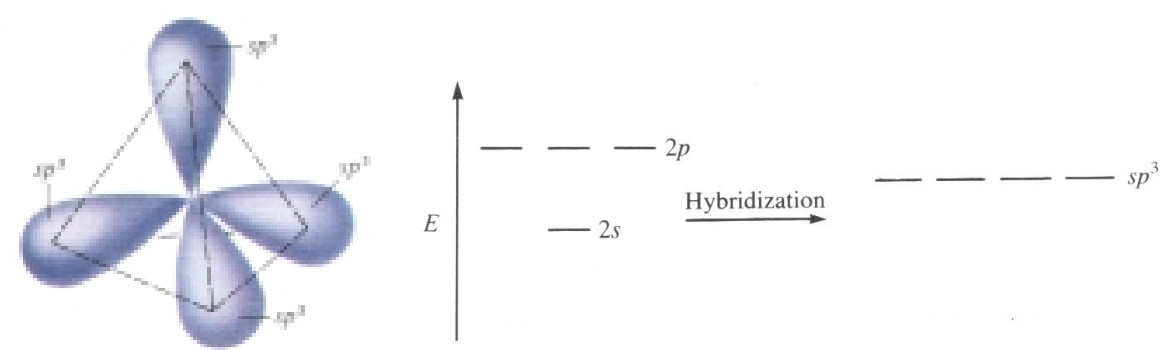

Figure 2.4 The tetrahedral set of four $s p^{3}$ orbitals formed from one $s$ and three $p$ orbitals.

For the III-V compounds (such as gallium arsenide), the atomic valence structures of Ga $\left(4 s^{2} 4 p^{1}\right)$ and As $\left(4 s^{2} 4 p^{3}\right)$ must each be hybridized to form bulk GaAs. The transfer of one electron from As to Ga allows both elements to adopt the $s p^{3}$ hybrid form of the valence band. This coordination of covalent bonds not only contributes to the tetrahedral structure of the bulk, but also dictates the configuration of atoms on the surface of semiconductors. In the bulk, the hybridized orbitals from each atom combine to form a bonding and antibonding molecular orbital. At the surface, some hybrid orbitals cannot form bonds, and partially filled $s p^{3}$ bonds, or "dangling bonds," remain [31]. 
Reducing the density of dangling bonds on the surface contributes to the electronic stability of surface species and consequently lowers the surface free energy. This is the driving mechanism for atomic rearrangement during surface reconstruction. Surface atoms have some freedom to move in directions that change their bond angles, thereby reducing the number of dangling bonds and altering their atomic positions. The tri-valent $\mathrm{Ga}$ atom prefers $s p^{2}$ bonding at the surface, which has a $120^{\circ}$ bond angle, as shown in Figure 2.5. The penta-valent As atom prefers the "native" atomic $s^{2} p^{3}$ bonding at the surface, which has an interbond angle of $94^{\circ}$. This bond rearrangement can potentially result in surface strain. Therefore, the most stable surface arrangement is the one that satisfies both steric and electronic considerations [32]. Steric means that no undue strain is imposed on the covalent bonds of the surface species. The relationship between the surface structure and the dangling bond states on the surface are examined in the next section.

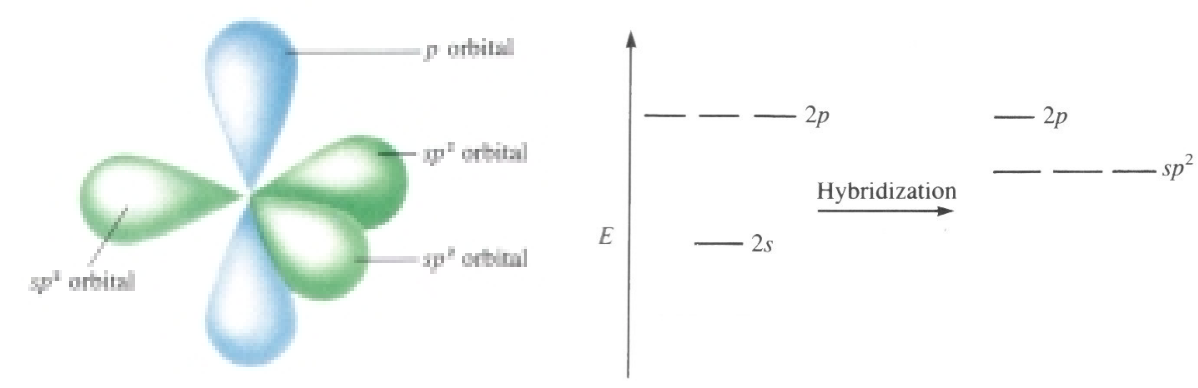

Figure 2.5 The $s p^{2}$ orbitals are formed from one $s$ and two $p$ orbitals. One $p$ orbital remains unchanged and is perpendicular to the plane of the hybrid orbitals.

\subsubsection{Atomic Surface Models and Surface phases}

The most commonly observed surface in MBE heteroepitaxial growth of III-V compounds is the GaAs (001). This surface has associated with it a large number of 
reconstructions in which the surface atoms reconfigure themselves to produce a periodicity different from that of the bulk crystal. These reconstructions are denoted using a convention defined by Wood [33]. The surface structure described by GaAs $(001)-(m \times n)$ is oriented with the [001] direction normal to the surface, and consist of a unit cell that is $m \times n$ times larger than the underlying bulk.

Under normal MBE growth conditions, the GaAs (001) surface exhibits a $(2 \times 4)$ reconstruction for an As-stabilized surface and a $90^{\circ}$ rotated $(4 \times 2)$ reconstruction for a Ga-stabilized surface. These structures arise because the surface atoms dimerize to reduce the density of dangling bonds. The unit cell for the $(2 \times 4)$ reconstruction has a two-fold and four-fold increase in periodicity in the [110] and [110] directions with respect to the bulk lattice spacing. The theoretical atomic structure for this reconstruction, known as the missing dimer row model, was initially proposed by Chadi [34] using a tight-binding based total-energy method. The model has been confirmed both experimentally by Frankel et al. [35] and by the scanning tunneling microscopy (STM) experiments of Pashley et al. [36] The proposed atomic surface model, shown in Figure 2.6, consists of a full atomic layer of Ga terminated with an atomic layer of As surface dimers, such that every fourth dimer is missing. This configuration, with one Asdimer vacancy per unit cell, was proposed under non-growing As-stabilized conditions on the basis that it results in the lowest-energy structure for the surface.

The GaAs (001) surface exhibits a large number of reconstructions that occur as the stoichiometry of the surface is varied. It has been observed that as the substrate temperature is increased, As desorption increases (i.e., the As chemical potential on the surface decreases). Under these conditions, the surface undergoes a sequence of 
structural transitions. These surface phases start from the most As-rich $c(4 \times 4)$ and progress through several different $(2 \times 4)$ phases. They then proceed through the $(4 \times 6)$ and $(3 \times 1)$ phases, and finally reach the $(4 \times 2)$ Ga-rich phase $[37,38]$.

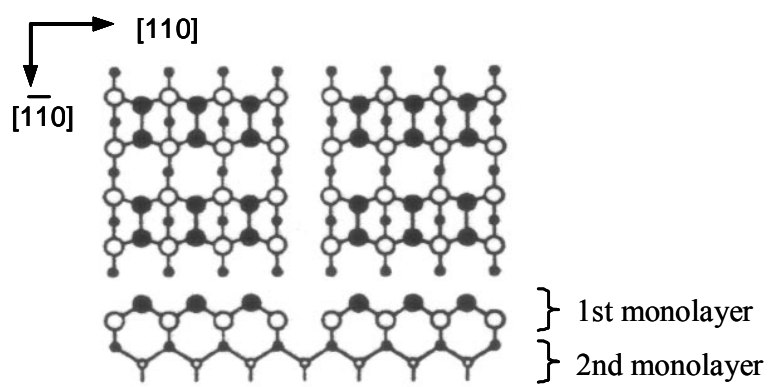

Figure 2.6 The atomic structure model for the GaAs $(001)-(2 \times 4)$ surface with three As dimers in the surface layer of the unit cell. Filled circles denote As atoms and open circles denote Ga atoms [34].

A surface phase diagram for the GaAs (001) reconstructions is provided in Figure 2.7, along with the atomic surface models for the $(2 \times 4)$ phases. These phases were classified by Farrell and Palmstrøm as the $(\alpha, \beta$, and $\gamma)$ phases of the $(2 \times 4)$ and have 0.5 , 0.75 , and $0.75+0.25$ atomic layers of As on the surface respectively [39]. The $\beta 2$ structure, initially proposed by Chadi [34], is defined by two As dimers and two dimer vacancies on the surface with one of the second layer Ga pairs removed. This structure has the same As coverage and almost the same surface-energy as the $\beta$ phase. STM experiments have shown that the $\beta$ and $\beta 2$ phases coexist on the surface under certain conditions [40]. It should be noted that two atomic layers, e.g., one Ga and one As, constitute an (001) monolayer. The lattice unit cell consists of two monolayers and consequently four atomic layers as depicted in the atomic surface models. While extensive information for the GaAs (001) surface phases exists, the present knowledge of 
these structures is still incomplete, and the study of surface mechanisms is dependent on the continued investigation of atomic surface structures.

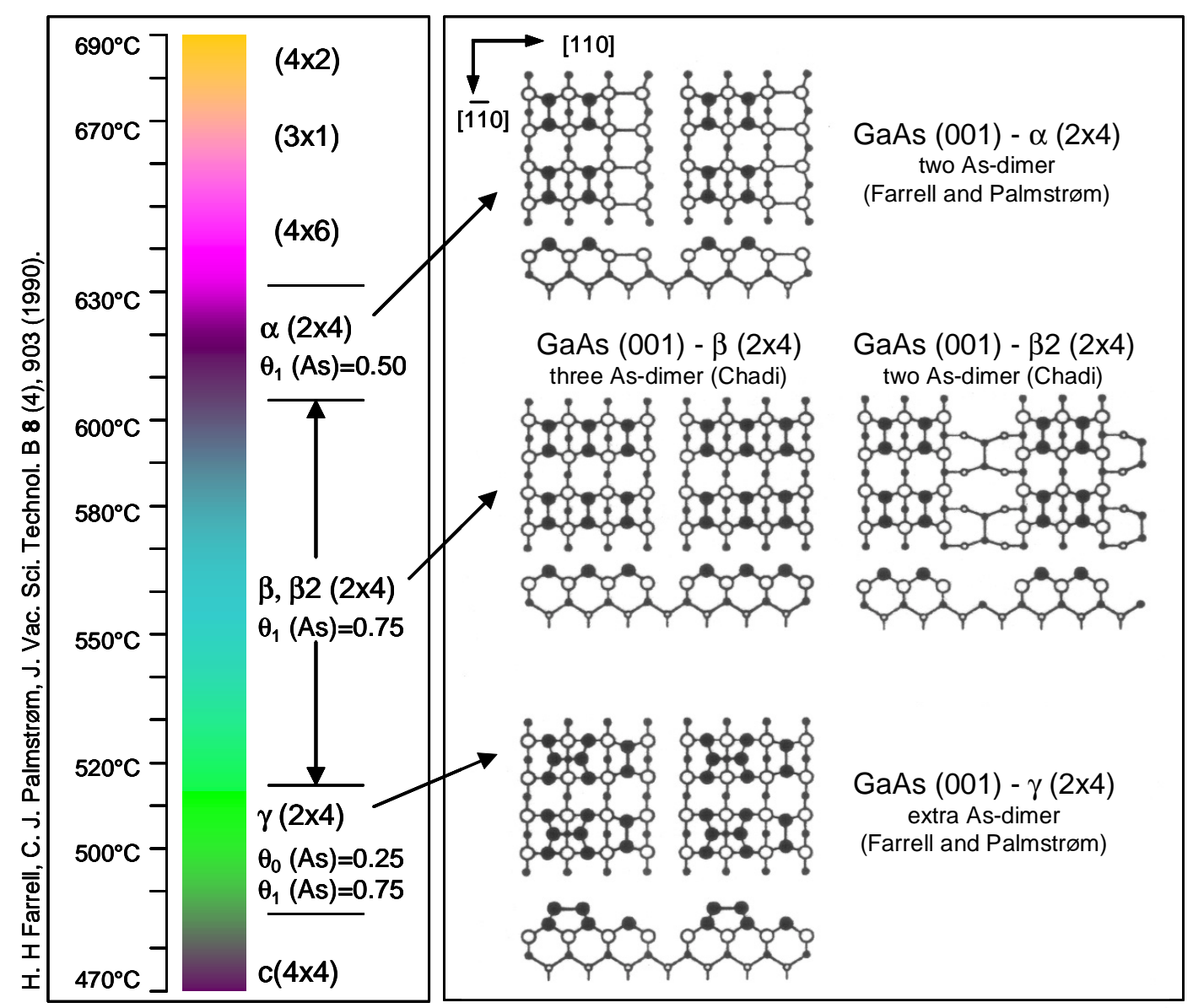

Figure 2.7 The surface phase diagram and surface reconstruction models for the GaAs (001) surface. Filled circles denote As atoms and open circles denote $\mathrm{Ga}$ atoms. 


\subsubsection{Electronic Stability Criterion}

The electronic stability criterion for the principal reconstructions of the GaAs (001) surface can be explained using the electron counting model (ECM). The ECM is the starting point from which atomic surface structures are proposed. The most prominent application of the ECM has been to narrow the possible atomic models for the III-V (001) surfaces $[34,41]$. The ECM states that the lowest-energy surface structure is obtained when the number of available electrons in the surface layer will exactly fill all dangling-bond states in the valence band of the material, leaving the dangling-bond states in the conduction band completely empty [31]. If this condition is satisfied, then the surface will be semiconducting and the band-gap at the surface will be close to that of the bulk. Otherwise, the partially filled dangling bonds may lead to a metallic surface.

The energy levels of dangling bonds can be estimated from the energy levels of the $s$ and $p$ atomic levels from which they are derived [42]. The dangling bond energy levels can then be compared with the conduction-band minimum and valence-band maximum of the bulk semiconductor. This is illustrated in Figure 2.8 for the GaAs (001) surface. The dangling-bond energy level of As lies below the valence-band maximum, whereas the dangling-bond energy level of Ga lies above the conduction-band minimum. Therefore, to satisfy the ECM, all the As dangling bonds should be filled and all Ga dangling bonds should be empty. This criterion was used to propose the atomic surface models in the previous section. It was also implemented to develop a coherent atomic growth model for the GaAs (001) surface [43]. A review of this growth model follows. 


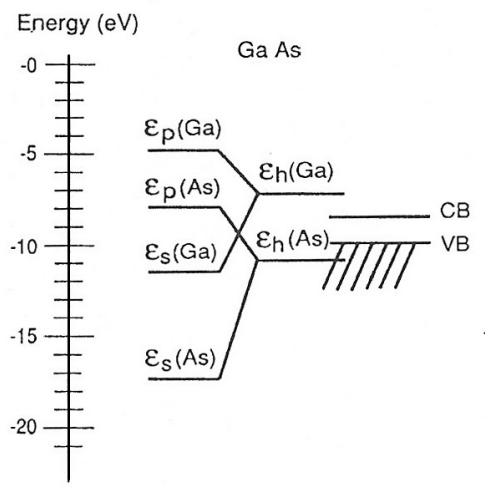

Figure 2.8 The energy levels of dangling bonds for the GaAs (001) surface [42].

\subsubsection{Atomic Growth Model and Growth Mechanisms}

The growth mechanisms that constitute the surface dynamics in growth by MBE on III-V substrates are complex. The growth process involves 16 atoms per unit cell for each monolayer. This large unit cell results in an enormous number of possible intermediate growth steps. The atomic growth model, developed by Harbison et al. [43] is fundamentally important for all III-V compounds. It is discussed here with respect to growth from the GaAs (001) surface. The following assumptions were made for the development of this atomic growth model:

(1) the dominant surface species at any point in the growth cycle must be electronically stable;

(2) the electronically unstable species will undergo chemical change or rearrange to form stable species;

(3) the surface atoms will tend to be dimerized, and surface dimer vacancies can occur;

(4) the physisorbed Ga is monatomic and the physisorbed As is diatomic; and

(5) the basic growth mechanisms are repeated for each monolayer. 
The model describes the MBE growth process in terms of four intermediate stages. Starting from the As-stabilized GaAs $(001)-\beta(2 \times 4)$ surface, these stages correspond to the chemisorption of $0.25,0.5,0.75$, and 1.0 of an atomic layer of Ga. Figure 2.9 illustrates the sequence of electronically stable intermediate structures determined for the growth model. The first stage corresponds to the arrival of Ga atoms at the surface, and the chemisorption of two adjacent Ga atoms across two of the three As dimers in the unit cell to form a Ga dimer. Alone, each $\mathrm{Ga}$ is electronically unstable. However, the formation of the Ga dimer bond stabilizes these species by forming an electronically stable configuration. In order for the second stage of the growth process to proceed, the As dimer vacancy in the first layer must be filled. The chemisorption of an As dimer to fill the vacancy and the chemisorption of a second pair of Ga atoms must occur essentially simultaneously within a given unit cell to achieve an electronically stable structure. This second stage results in 0.5 of an atomic layer of Ga at the surface. At this step, bonding sites for both $\mathrm{Ga}$ and As dimers become available on the surface. The chemisorption of Ga and As dimers to these available sites occurs during stage three. Each of these chemisorption steps individually satisfies the criterion for electronic stability. Due to the significantly larger As flux used during normal MBE growth, the chemisorption of As first is favored statistically. This third stage of the growth model results in 0.75 of an atomic layer of $\mathrm{Ga}$ at the surface. For the fourth stage of the growth model, there are no sites where either Ga or As dimers can individually be incorporated in an electronically stable fashion, much the same as the initial structure in stage two. As before, the correlated chemisorption of an As dimer and a pair of Ga atoms results in a stable surface structure and is determined to occur. This structure is characterized by a 
full atomic layer of $\mathrm{Ga}$ at the surface, and a new site for bonding an As dimer. This As chemisorption step is rapidly completed and the surface is now equivalent to the initial As-stabilized $\beta(2 \times 4)$ surface, completing the growth model.

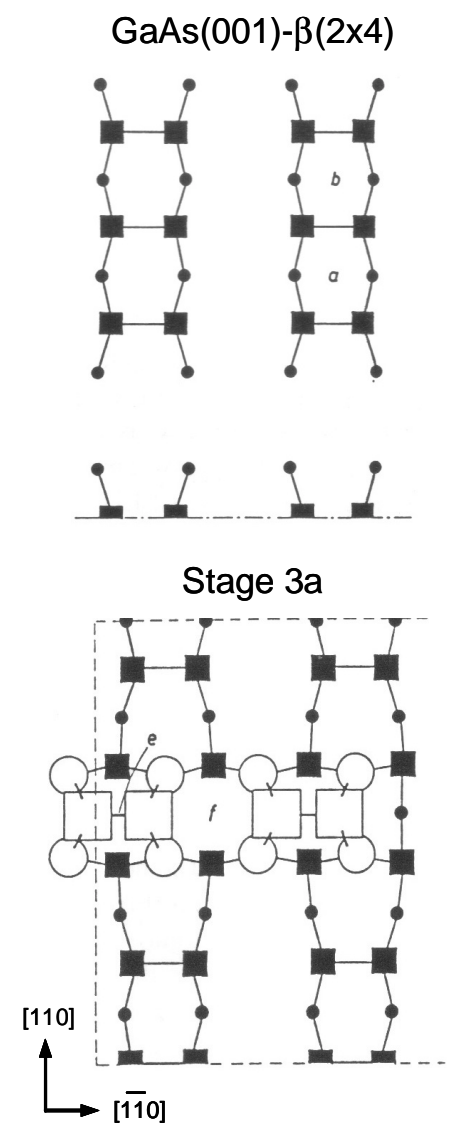

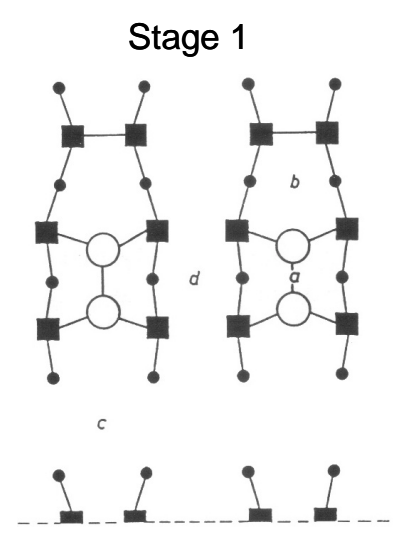

Stage $3 b$

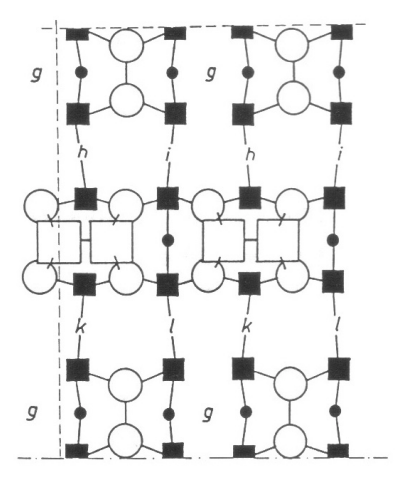

Stage 2

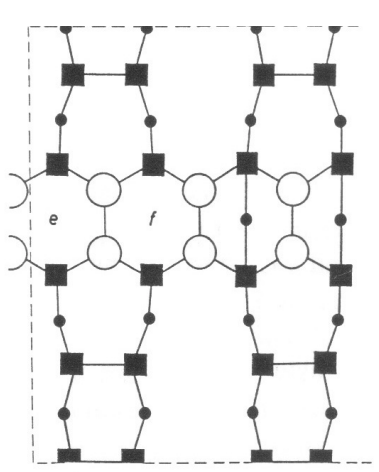

Stage 4

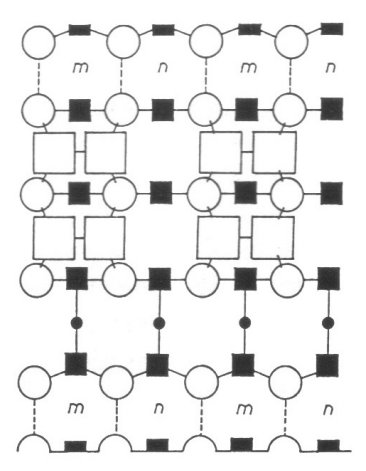

Figure 2.9 The sequence of electronically stable intermediate structures determined by the model for the complete monolayer growth cycle. The Ga atoms are indicated by $\circ$, and the As atoms are indicated by $\square$, with atoms closer to the surface indicated by larger symbols [43]. 


\subsubsection{Atomic Surface Structures}

As we have seen throughout this chapter, many of the effects resulting from microscopic processes have characteristic mechanisms that occur during MBE growth. The observation and interpretation of these mechanisms have been revolutionized by the advent of in-situ STM. This analysis technique has opened the way to obtaining realspace atomic-level information about surface morphology and has revealed unimagined levels of complexity in heteroepitaxial systems [44]. While in-situ STM was not available for this research effort, many of the results obtained by other research groups were invaluable in the determination of atomistic mechanisms observed, during this investigation of anion exchange. A collection of STM images and corresponding atomic surface structures for various reconstructions on both GaAs (001) and GaSb (001) is given in Appendix A.

\subsection{Summary}

The growth kinetics and growth dynamics of the MBE technique have been reviewed in this chapter. The theoretical models for crystal growth by MBE have been introduced, and the relationships between growth modes and surface morphology have been discussed. The occurrence of reconstruction at semiconductor surfaces has been considered, and its relevance for constructing atomic surface models and understanding growth mechanisms has been explored. The effects of anion exchange and other physical processes at the interfaces of mixed anion III-V heterostructures is discussed in the next chapter. 


\section{CHAPTER 3}

\section{ANION EXCHANGE}

The primary objective of this research was to characterize the dominant chemical and physical mechanisms that result in anion exchange. This research investigates the growth kinetics and the growth dynamics of anion exchange. Growth kinetics was examined through the use of a hybrid neural network that extracts kinetic parameters. Growth dynamics was determined through analysis of RHEED reconstructions. The focus of this chapter is to introduce the fundamental aspects of anion exchange and related processes in an effort to establish the experimental procedures necessary for the analysis of anion intermixing. The characterization techniques implemented during this research and the first-principles modeling used to investigate microscopic processes are the foundation for analysis.

\subsection{Anion Exchange \& Related Processes}

The dominant phenomena that have been recognized as causing anion intermixing at the interfaces of mixed anon III-V heterostructures are the segregation of excess stable $\mathrm{Sb}$ on the surface of As-based layers, As-for-Sb exchange in Sb-based layers, and phosphorus diffusion in both As and Sb-based layers. The theoretical and practical aspects of the physical processes observed during the course of this research are discussed in the following sections. 


\subsubsection{Anion Exchange}

During MBE growth of mixed anion materials, the sum of the incident group-V fluxes is typically in excess of the number of available group- $\mathrm{V}$ surface sites; therefore, the anions compete with each other for incorporation into the film. Anion intermixing at the interfaces of mixed anion III-V heterostructures occurs primarily due to anion exchange. The anion exchange reaction occurs when a dissimilar anion replaces or interchanges with a group- $\mathrm{V}$ atom that is already incorporated into the epitaxial layer. Several factors, such as surface reconstruction, microscopic strain, and surface morphology, have a significant impact on the extent to which anion exchange occurs.

The anion exchange process exhibits two distinct modes, observed by Xie et al. for $\mathrm{As}_{2}$ exposure of $\mathrm{GaSb}$, which are dependent on the substrate temperature and incident flux of the dissimilar anion [23]. For a low substrate temperature and a small anion flux, a group-V stabilized surface exposed to a dissimilar anion is depleted of its chemisorbed group-V atoms due to exchange. The anion exchange reaction, a classical Langmuir [45] type, occurs only in the first surface monolayer and results in the formation of a mixed anion alloy, hindering further exchange. The interfacial layer formed by this process is coherently strained, and the exchange process is said to be self-limiting. While significant anion intermixing does not occur for this mode of anion exchange, the presence of an unintended alloy at the interface can have a negative impact on interface properties. In the case of high substrate temperature and a large anion flux, exposure of a group- $\mathrm{V}$ stabilized surface to a dissimilar anion leads to a change in the surface morphology. The surface undergoes a lattice-mismatch strain sufficient enough to induce a 2-D to 3-D surface transition, which allows the dissimilar anion to exchange with 
group-V atoms on the sub-surface. This type of surface transition results in coherent 3-D clusters and is thought to be similar to the strain induced island formation that occurs during the growth of InAs/GaAs quantum dots [27]. Consequently, this anion exchange mode results in a considerable amount of anion intermixing and interface roughening, significantly degrading the electronic properties of the material. It should be noted that a strong correlation between these anion exchange modes and surface reconstruction is present. Interface roughness and layer composition for mixed anion III-V heterostructures are difficult to control principally because the anion exchange process usually occurs, often in conjunction with other processes.

\subsubsection{Segregation}

The growth of mixed anion III-V heterostructures often exhibits surface segregation of one of the anion species. Surface segregation results in anion intermixing at the interface primarily due to segregant incorporation during subsequent layer overgrowth. In the case of surface segregation on bulk binary alloys, segregation occurs at thermodynamic equilibrium and is determined by the sign of the segregation energy. Segregation energy is defined as the energy difference between a state with the segregant at the surface and that with the segregant incorporated in the bulk [46].

In an effort to predict segregation, several theories for estimating segregation energy have been proposed. In principle, surface segregation energy is approximated using factors such as surface energy, binding energy between atoms, and strain energy due to the segregant atom. One method for predicting segregation indicates that the element with the lower surface energy segregates on the surface in equilibrium. During MBE growth of mixed anion materials, the surface on which the segregation mechanism 
occurs is not always at equilibrium; therefore, these theories have limited use. Recent studies of segregation behavior indicate that adsorption energy can be used to predict surface segregation under non-equilibrium conditions [47]. Alternatively, the segregation mechanism does exhibit several characteristic features that may be studied in an effort to further the understanding of this process as it relates to the growth of mixed anion materials. When an element segregates on the surface, the surface concentration of the segregant saturates. The concentration of the segregant at saturation is independent of substrate temperature. For extended exposure times after the segregant saturates, interfacial reactions such as diffusion and exchange may occur if sufficient kinetic energy is provided. The saturation behavior of the segregation process is thought to be caused by a stabilization mechanism associated with the surface free energy. For mixed anion materials, the anions segregating on the surface are gradually incorporated during growth of the next layer. This leads to anion intermixing, with a decreasing segregant composition in the growth direction. Surface segregation results in the formation of coherently strained layers and a graded interface profile, which leads to interface smoothing and modification of the electronic properties of the material.

\subsubsection{Diffusion}

There are two potential diffusion mechanisms that can contribute to anion intermixing at the interfaces of mixed anion III-V heterostructures. These atomic diffusion mechanisms, defined as substitutional and interstitial diffusion, are illustrated for a two-dimensional lattice in Figure 3.1. In the case of substitutional diffusion, the dissimilar anion moves between available lattice sites in the zincblende structure. Specifically, the anion "substitutes" for an atom in the group-V sublattice. In order for 
the substitutional diffusion process to occur, vacancies in the lattice must be present. Theoretically, a finite number of lattice vacancies are always present in semiconductors. During MBE growth at high substrate temperatures, vacancies may also be created due to desorption of the group-V element. In general, substitutional diffusion proceeds at a relatively slow rate because the number of vacancies in the group- $\mathrm{V}$ sublattice is limited. This slow diffusion rate can be advantageous with respect to controlling anion intermixing. For the interstitial diffusion mechanism, the dissimilar anion diffuses through the crystal by moving into the vacant or "interstitial" space between lattice sites. This diffusion mechanism does not require the presence of lattice vacancies. At the atomic scale, a considerable space exists between atoms in the zincblende structure. Interstitial diffusion, therefore, proceeds much more rapidly than substitutional diffusion. This rapid diffusion rate leads to anion intermixing that is difficult to control. It should be noted that only atoms that occupy lattice sites contribute to the carrier transport properties of the material. Consequently, substitutional diffusion directly affects the electronic and optical properties of mixed anion heterostructures, whereas interstitial diffusion often leads to structural defects, which can degrade overall crystalline quality.
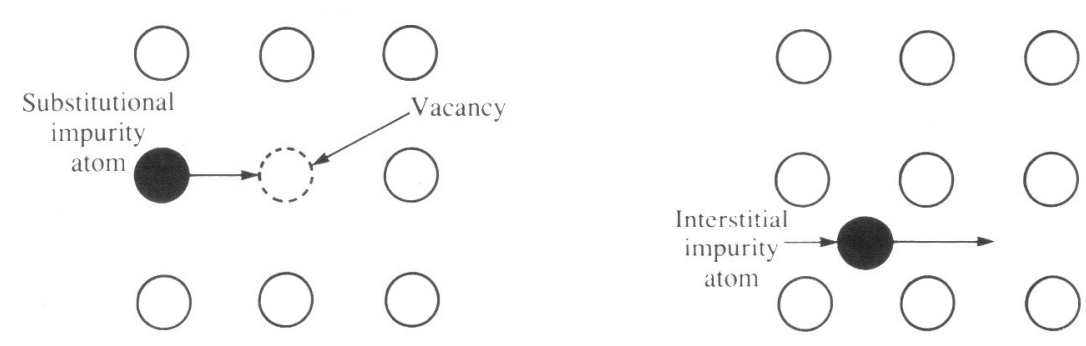

Figure 3.1 The atomic diffusion mechanisms in a 2-D lattice. For substitutional diffusion (left), the dissimilar anion moves among lattice vacancies. For interstitial diffusion (right), the dissimilar anion moves into the space between lattice sites. 


\subsection{Experimental Technique}

The investigation of microscopic processes occurring at the interfaces of mixed anion III-V heterostructures grown by MBE requires an experimental technique that extracts detailed information from the sample set. Traditional experimental techniques implement a "single-variable" investigation approach that explores a group of variables

one at a time. This research effort employs a statistical experimental design technique capable of varying the parameters of interest simultaneously and systematically.

\subsubsection{Design of Experiments for III-V Heterostructures}

Statistical experimental design is an organized method of conducting experiments capable of extracting the maximum amount of information from a limited number of experiments. The essential issues in experimental design are the selection of the parameters to be varied and the determination of the parameter space over which the variation results in a significant and measurable response. The number of parameters used to design a statistical experiment has a direct influence on the number of experimental candidates (trials). This criterion ultimately determines the overall impact on system resources.

The designed experiment developed for each material system in this research effort was implemented using a D-optimal design technique [48]. In contrast to standard classical designs, such as factorials and fractional factorials, D-optimal designs allow the presence of qualitative parameters and a constrained or irregularly shaped design space. The D-optimal method is based on the optimization of the determinant calculated from the covariance matrix of the candidates. In order to obtain a model of high quality, the D-optimal design chooses the candidates that maximize the determinant of the covariance 
matrix. This optimality criterion results in minimizing the generalized variance of the estimated model coefficients for a pre-specified model.

This research uses the $R S /$ Discover statistical software package, which employs the Galil-Kiefer [49] sequential search algorithm to produce D-optimal designs. Once the responses of interest have been measured for each experimental trial, the results may be modeled using a response surface. The response surface method is an empirical approach that is widely used to model complex systems. While this method has the ability to establish a model, this research also explores the use of neural networks to enhance the modeling of anion exchange for MBE growth of mixed anion heterostructures.

\subsubsection{Superlattice Structures for Analysis}

The development of structures that exhibit the dominant MBE growth processes is crucial to the analysis of anion exchange. Two types of mixed anion heterostructures were investigated during the course of this research. The initial experiments, designed to study anion intermixing, used lattice-matched structures composed of a mixed cation ternary grown epitaxially on a binary alloy having a dissimilar anion. These structures, illustrated in Figure 3.2, are designated as the $\mathrm{III}_{\mathrm{A}}-\mathrm{III}_{\mathrm{B}}-\mathrm{V}_{\mathrm{A}} / \mathrm{III}-\mathrm{V}_{\mathrm{B}}$ heterostructures and are composed of heterointerfaces grown in both the normal and inverted interface configuration. A growth interrupt is used at the interface of these structures to allow the dissimilar anion to impinge on a non-stabilized surface, resulting in anion exchange. The experiment for these initial investigations was designed such that the heterointerfaces corresponding to the experimental trials could be grown continuously on a single substrate with buffer layers separating each of them. As a result, a superlattice structure was formed during growth, with the advantage that only one MBE growth run (i.e., 
growth of a single SL structure) was required for an entire experimental design. It should be noted that these structures consisted of mixed anions and mixed cations across the interface, and group-III intermixing was also found to occur, thus complicating the analysis of anion exchange.

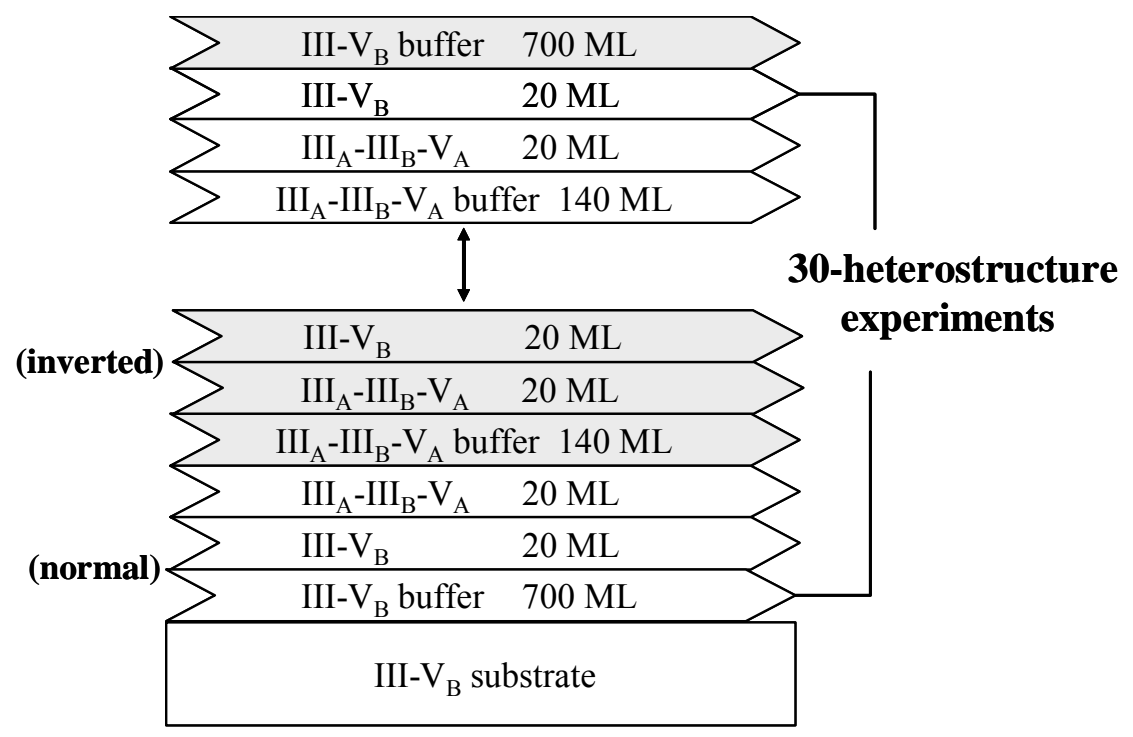

Figure 3.2 The basic SL structure designed to study anion exchange using the $\mathrm{III}_{\mathrm{A}}-\mathrm{III}_{\mathrm{B}}-$ $\mathrm{V}_{\mathrm{A}} / \mathrm{III}-\mathrm{V}_{\mathrm{B}}$ heterostructures grown in both the normal (white) and inverted (gray) interface configuration.

The subsequent experiments, designed to study anion intermixing, used structures developed specifically for anion exchange analysis. These structures were formed by allowing a dissimilar anion flux to impinge on a static group-V stabilized binary alloy surface. Therefore, in these experiments, exchange was induced using a non-growing (no incident group-III) anion exposure technique. The resulting structures, illustrated in Figure 3.3 , consist of a mixed anion ternary coherently strained to the binary alloy surface. These structures are designated as the $\mathrm{III}-\mathrm{V}_{\mathrm{A}}-\mathrm{V}_{\mathrm{B}} / \mathrm{III}-\mathrm{V}_{\mathrm{A}}$ heterostructures and consist of only mixed anions across the interface. In an effort to examine both the 
normal and inverted interface configuration, the exposure of the group-V stabilized surface to the dissimilar anion was followed by overgrowth using the binary alloy. This procedure was repeated up to twenty times on a single substrate in order to determine the potential variation of the exchange process. The complete growth sequence results in the formation of a strained layer superlattice (SLS) structure. The experiment for these investigations required an MBE growth run (i.e., growth of a SLS structure) for each experimental trial.

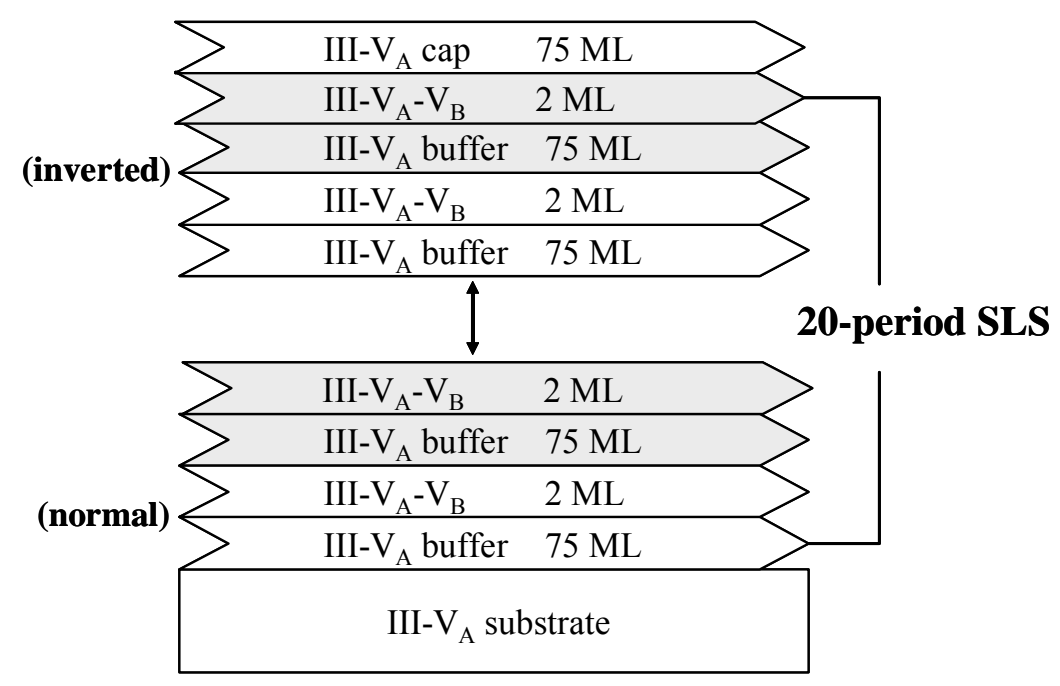

Figure 3.3 The SLS structure designed to study anion exchange using the III- $\mathrm{V}_{\mathrm{A}}-\mathrm{V}_{\mathrm{B}} / \mathrm{III}-$ $\mathrm{V}_{\mathrm{A}}$ heterostructures. The structure consists of a mixed anion ternary coherently strained to the binary alloy surface.

\subsection{Characterization of Anion Exchange}

The superlattice structures designed for analysis of anion exchange were characterized using several in-situ and ex-situ compound semiconductor analysis techniques. The thin-film characterization techniques used during the course of this research are discussed in the following sections. 


\subsubsection{Reflection High-energy Electron Diffraction}

Reflection high-energy electron diffraction (RHEED) is widely used for surface kinetic studies during MBE growth of III-V materials. In MBE monitoring using RHEED, high-energy (10-40 keV) electrons are incident on the substrate at a variable glancing angle $\left(1-3^{\circ}\right)$. This small incidence angle makes RHEED a surface-sensitive technique [50]. The incident electrons undergo diffraction at the surface according to the Bragg law and are scattered onto a phosphorescent screen positioned directly opposite the RHEED gun in the growth chamber. The phosphor screen then contains the twodimensional RHEED pattern, which corresponds to rows of atomic periodicity on the surface. It is possible to obtain information on surface structure, microstructure, and smoothness from RHEED patterns. The RHEED geometry is ideal for MBE as the electron gun and phosphorescent screen are positioned remotely from the substrate, so that they do not interfere with the growth process and are not exposed directly to an evaporation source or to the broad face of a hot substrate.

\subsubsection{High-resolution X-ray Diffraction}

MBE grown III-V heterostructures are often characterized nondestructively using high-resolution x-ray diffraction (HRXRD). This analysis technique provides a means of investigating the detailed structure of semiconductor materials. HRXRD is very sensitive to differences in strain and inter-planar tilts due to the high angular resolution obtainable. Diffraction for this method is due essentially to the existence of certain phase relations [8]. This makes HRXRD an ideal semiconductor analysis technique for characterizing the composition and strain of thin films or epitaxial layers. 
Triple-axis x-ray diffraction is an extension of high resolution x-ray diffraction in which an analyzer crystal is placed in front of the detector in order to restrict its angular acceptance. This configuration allows separation of the effects of strains and tilts in the material, and in reciprocal space a small volume is defined that is sampled by the detector at a given setting. Thus, complete two-dimensional reciprocal-space maps (RSMs) may be obtained, giving very detailed information on thin epitaxial layers and multilayer structures.

\subsubsection{X-ray Photoelectron Spectroscopy}

Angle-resolved x-ray photoelectron spectroscopy (XPS) is a near surface semiconductor analysis technique. The advantage of this technique is that large chemical shifts in peak position dependent on the surface chemical composition can be observed. This makes it very useful for the study of isoelectronic compounds often formed on the surface of a binary semiconductor material during exposure by a dissimilar anion. The use of XPS in determining the chemical composition of mixed anion III-V interfaces was explored in this research.

\subsubsection{High-resolution Transmission Electron Microscopy}

High resolution transmission electron microscopy (HRTEM) is used for determination of structural properties. This technique is based on the diffraction of electrons as they pass through a very thin film or epitaxial layer. There are two basic modes of TEM operation. High resolution imaging is usually performed in the bright field mode, for which the (000) transmitted beam contributes to the image [50]. The size of the objective aperture in bright-field mode directly determines the information to be 
emphasized in the final image. It should be noted that for HRTEM, the sample preparation thinning process is generally destructive.

\subsubsection{Atomic Force Microscopy}

Atomic force microscopy (AFM) is an invaluable tool for characterization of semiconductor films. The basic technique is that of a high-resolution profilometer. AFM is used to determine surface micro-roughness for semiconductor materials, with angstrom-level precision. The desired surface is scanned in a raster pattern, and the data is reconstructed in order to obtain a nanometer-scale topographic image. This technique has the ability to image both insulating and conducting surfaces.

\subsection{Semi-Empirical Modeling of Anion Exchange}

The investigation of the growth kinetics of anion exchange was accomplished by implementing a semi-empirical (or "hybrid") modeling technique. Semi-empirical modeling incorporates the best aspects of fundamental physical modeling and purely empirical modeling methods. For the MBE growth of mixed anion heterostructures, the physical model provides the essential relationships between the growth conditions and the compositional profiles at the interfaces. The physical model describes the MBE growth mechanisms through the use of expressions derived from first principles. These expressions consist of reaction rate constants, which serve as fitting parameters by providing a means of generalizing the physical model to the growth process. The empirical model provides a method for determining the reaction rate constants, using statistically designed experiments that characterize the process responses. The statistical experimental design technique implemented for this research provides a basis for 
developing models of anion exchange. This section introduces a quantitative physical model based on first order kinetic theory and uses this model to design a semi-empirical model of anion exchange based on hybrid neural networks.

\subsubsection{Kinetic Modeling}

The development of a model that provides quantitative considerations of kinetics and identification of the microscopic processes occurring during MBE growth is of great significance for the advancement of mixed anion materials. The MBE growth models that currently exist provide only semi-quantitative analysis of growth kinetics and tentative identification of processes [51,52]. The majority of these models are unable to determine the dependence of the composition of a ternary alloy on the growth parameters, particularly in the case of mixed anion materials. These models provide only basic qualitative descriptions of changes in the growth rate or decomposition rate with respect to substrate temperature, and even simplified models require very complex calculations.

The kinetic model developed for this research effort examines several processes occurring at the interfaces of mixed anion heterostructures, including anion exchange, surface desorption, surface segregation, and diffusion. The model can be used to determine the contribution of each of these microscopic processes to the composition of the dissimilar anion at the interface as a function of substrate temperature, flux of the impinging anion, and anion exposure time. The analytical expressions for anion exchange and segregation used to develop this kinetic model are based on work performed by Bandić et al. [53] and have been modified for the purposes of this research to incorporate an extended set of growth processes. In an effort to obtain a physical understanding of these processes, the first order kinetic model explicitly incorporates 
three primary phases of the growth sequence for the SLS structures: (1) anion exposure; (2) binary alloy overgrowth; and (3) group-V surface stabilization, as described in the following sections.

\subsubsection{Anion Exposure}

The first phase of mixed anion heterostructure formation for the SLS structures involves the exposure of a static group-V stabilized binary alloy surface to a dissimilar anion. The microscopic processes, which have been determined to occur during this phase of the growth sequence, include anion exchange at the surface, surface desorption, and diffusion of the dissimilar anion. These processes occur in the absence of a group-III flux (i.e., non-growing conditions). Therefore, these microscopic processes are governed by the reconstruction of the group-V stabilized surface and the surface composition of the impinging dissimilar anion. Taking this into account and assuming that no nucleation occurs, a first-order rate equation describing the dynamics of the change in surface concentration $y$ of the dissimilar anion is defined using the equation

$$
\frac{d y}{d t}=s \Phi(1-y)-\frac{y}{\tau_{d}}
$$

The first term in this equation, $s \Phi(1-y)$, represents the increase in the surface concentration of the dissimilar anion on the group- $\mathrm{V}$ stabilized surface due to anion exchange. This process is proportional to the flux $\Phi\left(\right.$ molecules $\left./ \mathrm{cm}^{2} \cdot \mathrm{s}\right)$ of the impinging dissimilar anion and the surface coverage $(1-y)$ of the native group- $\mathrm{V}$ element, which is determined by the static surface reconstruction. If we assume that the group-V stabilized surface reconstruction contains vacancies, then the surface coverage can be approximated as the fraction of native group- $\mathrm{V}$ surface sites available to be exchanged with the 
dissimilar anion. It should be noted that an overall efficiency factor $s\left(\mathrm{~cm}^{2}\right)$ is used to model the probability of the exchange reaction proceeding. This factor provides an estimate of the sticking coefficient of the dissimilar anion and may depend on surface chemistry, strain, and substrate temperature. Therefore, the sticking coefficient is one of the primary fitting parameters for the semi-empirical model. The second term in this equation, $-y / \tau_{d}$, represents the decrease in the surface concentration of the dissimilar anion due to surface desorption. This process is modeled in terms of the reaction rate constant, $\tau_{d}^{-1}=\tau_{0 d}^{-1} \exp \left(-E_{d} / k_{B} T_{s}\right)$, where $\tau_{0 d}^{-1}($ molecules/s) is the reaction frequency and $E_{d}(e V)$ is the activation energy. The solution to Equation (3.1) for the exposure of a group- $\mathrm{V}$ stabilized surface to a dissimilar anion is expressed as

$$
y\left(T_{s}, t_{\exp }, \Phi\right)=s \Phi \tau\left[1-\exp \left(-t_{\exp } / \tau\right)\right]
$$

where $\tau \equiv\left(\tau_{d}^{-1}+s \Phi\right)^{-1}$, and $t_{\exp }$ is the exposure time of the impinging dissimilar anion. This equation describes the surface concentration $y$ (mole fraction) of the dissimilar anion due to the microscopic processes of anion exchange and surface desorption for the initial phase of the growth sequence. It should be noted that in the limit of high dissimilar anion flux $(\Phi \rightarrow \infty)$ or low substrate temperature $\left(T_{s} \rightarrow 0\right)$ such that desorption rate $\tau_{d}^{-1}$ is negligible, this equation is reduced to $y=\lim _{\substack{\Phi \rightarrow \infty \\ T S \rightarrow 0}}\left(1-\exp \left(-t_{\exp } / \tau\right)\right)$, and $y \rightarrow 1$ is obtained. This indicates that the anion exchange process is expected to fully convert the group- $\mathrm{V}$ stabilized surface in the steady state.

In order to completely model the first phase of the growth sequence, the diffusion process must also be considered. When the group- $\mathrm{V}$ stabilized surface becomes fully converted by the anion exchange process, a steady state is reached, and diffusion of the 
dissimilar anion into the bulk binary alloy may occur. The diffusion process follows Fick's second law of diffusion, and is analytically modeled in terms of the concentration $N$ of the diffused dissimilar anion, using the rate equation

$$
\frac{\partial N}{\partial t}=\frac{D \partial^{2} N}{\partial x^{2}}
$$

where $D=D_{0} \exp \left(-E_{a} / k_{B} T_{s}\right)$ is the diffusion coefficient, and $x$ is the diffusion length. This partial differential equation is solved using the boundary condition defined by the constant-source diffusion model. Constant-source diffusion requires that the surface concentration be held constant throughout the diffusion process. When this boundary condition is satisfied, the solution to Equation (3.3) for the diffusion of a dissimilar anion into a bulk binary alloy is expressed as

$$
N\left(x, t_{\text {exp }}\right)=N_{0} \operatorname{erfc}\left(x / 2 \sqrt{D t_{\text {exp }}}\right)
$$

where $N_{0}$ is the dissimilar anion concentration at the surface. This equation describes the concentration of the dissimilar anion for the layer at diffusion length $x$. The constantsource diffusion process results in a complementary error function (erfc) distribution of the dissimilar anion in the bulk. Consequently, the total contribution of the diffusion process to the concentration of the dissimilar anion in the bulk binary alloy is defined as the dose $Q$, and is determined by integrating Equation (3.4).

$$
Q=\int_{0}^{\infty} N\left(x, t_{\exp }\right) \cdot d x=2 N_{0} \sqrt{D t_{\exp } / \pi}
$$

It should be noted that the dissimilar anion concentration $N_{0}$ at the surface is equivalent to the steady state value of the surface concentration described by Equation (3.2). Therefore, the final result for the concentration of the dissimilar anion due to the 
microscopic processes, which occur during the first phase of the growth sequence, is expressed as

$$
y\left(T_{s}, t_{\exp }, \Phi\right)=s \Phi \tau\left[1-\exp \left(-t_{\exp } / \tau\right)\right] \times\left[1+2 \sqrt{D t_{\exp } / \pi}\right\rfloor
$$

This result is combined with the surface segregation modeling in the next section in order to obtain a kinetic model for anion intermixing.

\subsubsection{Binary Alloy Overgrowth}

The second phase of mixed anion heterostructure formation for the SLS structures involves the overgrowth of the anion exposed surface using the binary alloy. The microscopic processes, which have been determined to occur during this phase of the growth sequence, include surface segregation and surface desorption of the dissimilar anion. These processes occur during MBE growth, due to the presence of the dissimilar anion at the interface following the anion exposure phase. The surface segregation

process is modeled in terms of the reaction rate constant, $\tau_{s}^{-1}=\tau_{0 s}^{-1} \exp \left(-E_{s} / k_{B} T_{s}\right)$, where $\tau_{0 s}^{-1}\left(\right.$ molecules/s) is the reaction frequency, and $E_{s}(\mathrm{eV})$ is the activation energy. This process contributes to the gradual incorporation of the dissimilar anion into the growing layers. In contrast, the surface desorption process represents a net decrease in the dissimilar anion concentration. The reaction rate constant $\tau_{d}^{-1}$ is used to model this process consistent with the previous section. These reaction rate constants are used to model the concentration $S_{n}(t)$ of the dissimilar anion for monolayer $n$, during binary alloy growth, where the concentration of the initial anion exposed surface is normalized to be unity. It should be noted that since both surface segregation and surface desorption processes occur during binary alloy growth, the dissimilar anion concentration for each 
layer $n$ decays exponentially and is defined as $S_{n}(t)=S_{n}(0) \exp \left(-t / \tau_{\|}\right)$, where $\tau_{\|}^{-1} \equiv\left(\tau_{s}^{-1}+\tau_{d}^{-1}\right)$. Using this expression, the relationship between the dissimilar anion concentration of monolayers $n$ and $n+1$ is derived for the growth interval $0 \leq t \leq t_{M L}$, and modeled using the equation

$$
S_{n+1}(0)=S_{n}(0)\left[1-\exp \left(-t_{M L} / \tau_{\|}\right)\right] \frac{\tau_{d}}{\tau_{s}+\tau_{d}}
$$

where $t_{M L}$ is the time required to grow a single monolayer of the binary alloy, i.e., reciprocal growth rate $(\mathrm{ML} / \mathrm{s})$. It can be shown that $S_{n}(0)$ represents geometrical progression. As a result, this equation describes the fraction of the dissimilar anion lost from layer $n$, which segregates rather than desorbs and contributes to the dissimilar anion concentration of the next surface layer. Similarly, the fraction of the dissimilar anion concentration lost from layer $n$ due to desorption is expressed as

$$
\delta_{n}=S_{n}(0)\left[1-\exp \left(-t_{M L} / \tau_{\|}\right)\right] \frac{\tau_{s}}{\tau_{s}+\tau_{d}}
$$

The expressions, derived in Equation (3.7) and Equation (3.8), are used to determine the concentration of the dissimilar anion during binary alloy growth. The net decrease in the dissimilar anion concentration, due to the surface desorption process is derived by taking the sum of $\delta_{n}$ over the layers grown. It should be noted that the surface desorption process is the only mechanism that reduces the dissimilar anion concentration during binary alloy growth. Therefore, the total dissimilar anion concentration incorporated due to the surface segregation process is simply $R=1-\sum_{n=0} \delta_{n}$, and can be expressed as 


$$
R=\frac{\tau_{s}+\tau_{d}}{\tau_{d}+\tau_{s} \exp \left(t_{M L} / \tau_{\|}\right)}
$$

This equation is the final result for the concentration of the dissimilar anion due to the microscopic processes occurring in the second phase of the growth sequence. Combining this equation with the anion exposure result in Equation (3.6), the completed kinetic model is obtained and expressed as

$$
y\left(T_{s}, t_{\exp }, \Phi\right)=s \Phi \tau\left[1-\exp \left(-t_{\exp } / \tau\right)\right] \times\left[\frac{\tau_{s}+\tau_{d}}{\left(\tau_{d}+\tau_{s} \exp \left(t_{M L} / \tau_{\|}\right)\right)}+2 \sqrt{D t_{\exp } / \pi}\right]
$$

The kinetic model developed for this research effort quantitatively describes the complete growth sequence for the SLS structures. This model identifies the contributions of the microscopic processes of anion exchange, surface desorption, diffusion, and surface segregation and can be used to determine the composition of the dissimilar anion at the interfaces of mixed anion heterostructures. In order to accurately implement this model, several unknown constants or fitting parameters must be determined. The parameters for this model are the efficiency factor or sticking coefficient $s$, the diffusion coefficient and its activation energy $D_{0}, E_{a}$, the surface desorption coefficient and its activation energy $\tau_{0 d}^{-1}, E_{d}$, and the segregation coefficient and activation energy $\tau_{0 s}^{-1}, E_{s}$. The estimation of these parameters is addressed later in this chapter by designing a hybrid neural network semi-empirical model.

\subsubsection{Neural Network Modeling}

Neural networks have recently shown success for semiconductor applications requiring models of dynamic physical processes [54]. This is primarily due to the fact that neural networks possess the unique capability of learning complex nonlinear 
relationships, even for noisy input and output patterns. In principle, neural networks are able to resolve arbitrary nonlinear mappings via massively parallel interconnection of simple computational elements or processing units, called "neurons". In the standard "black-box" configuration, a neural network consists of multiple layers of these elementary processors. Individual neurons are interconnected in such a way that the information necessary to model the desired process is stored in the weight of the connections between them. The functionality of each neuron is to compute the weighted sum of its inputs and determine its activation level using a non-linear sigmoidal function, such as the hyperbolic tangent. This activation function enables neural networks to generalize with a degree of freedom not available in statistical regression techniques [55].

The neural network model used for this research is a multilayer feed-forward neural network. The basic structure for this type of network is illustrated in Figure 3.4. The most common method of learning for feed-forward neural networks is the error backpropagation (BP) algorithm. This algorithm is classified as a supervised learning technique and requires that the network be trained using a set of input/output data, whose functional relationship the network attempts to learn. Network learning is designed to determine an appropriate set of network weights that facilitate mapping the desired process. For the standard BP algorithm, these weights are initially random. An input vector is presented to the network, and the network outputs are determined by propagating the inputs through the layers of the network. The output of the final layer is compared to the desired or measured output data, and the squared difference between the two vectors determines the network error. This network error is then propagated backward through the network, and learning occurs by minimizing the error via 
modification of the weights one layer at a time. The mathematical approach for error minimization is known as gradient descent, which adjusts the network weights by an amount proportional to the derivative of the error with respect to the previous weights. After the network weights are updated, the procedure is repeated for the set of input/output data until convergence is achieved.

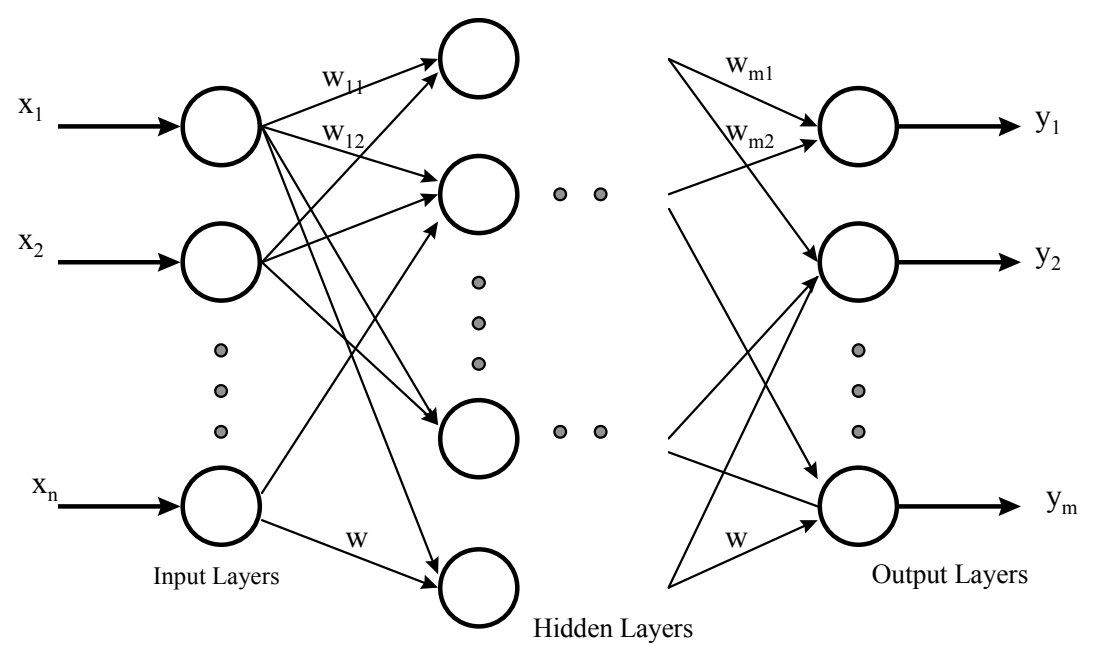

Figure 3.4 The basic structure for the multilayer feed-forward neural network showing input, hidden, and output layers.

Neural network models provide advantages in both accuracy and robustness over statistical models. This research implements a back-propagation neural network model for a preliminary investigation of the microscopic processes that contribute to anion intermixing at the interfaces of mixed anion III-V heterostructures. Unfortunately, standard "black-box" neural networks offer limited insight into understanding the underlying physical process. This shortcoming was addressed by developing a hybrid neural network which incorporates partial knowledge regarding the first principles relationships inherent in the process being modeled. 


\subsubsection{Hybrid Neural Network Design}

The hybrid neural network model developed for this research effort incorporates the first principles kinetic model, expressed in Equation (3.10), with a back-propagation neural network. The first principles kinetic model specifies the interactions of the microscopic processes from physical considerations, while the neural network complements this model by estimating unknown or unmeasured process parameters in such a way as to completely satisfy the first principles relationships. It should be noted that the kinetic model is always consistent with first principles; therefore, the generalization and extrapolation performed by the neural network is confined only to the unmeasured parameters of the process, preventing non-physical predictions [56].

The structure of the hybrid neural network model is illustrated in Figure 3.5. The method of learning implemented for this hybrid neural network is a modified error backpropagation algorithm that also requires the network be trained using a set of input/output data. The neural network component of the hybrid implementation has the MBE process conditions and the experimental dissimilar anion composition as its inputs. The outputs of the neural network component are the unknown parameters, required to implement the kinetic model. Using these estimated process parameters, the kinetic model component of the hybrid neural network can predict the dissimilar anion composition $\left(y_{p}\right)$. This predicted composition is the final output of the hybrid neural network model. It should be noted that network learning for this modeling technique is designed to determine an appropriate set of network weights for the neural network component that facilitate process parameter estimation. 


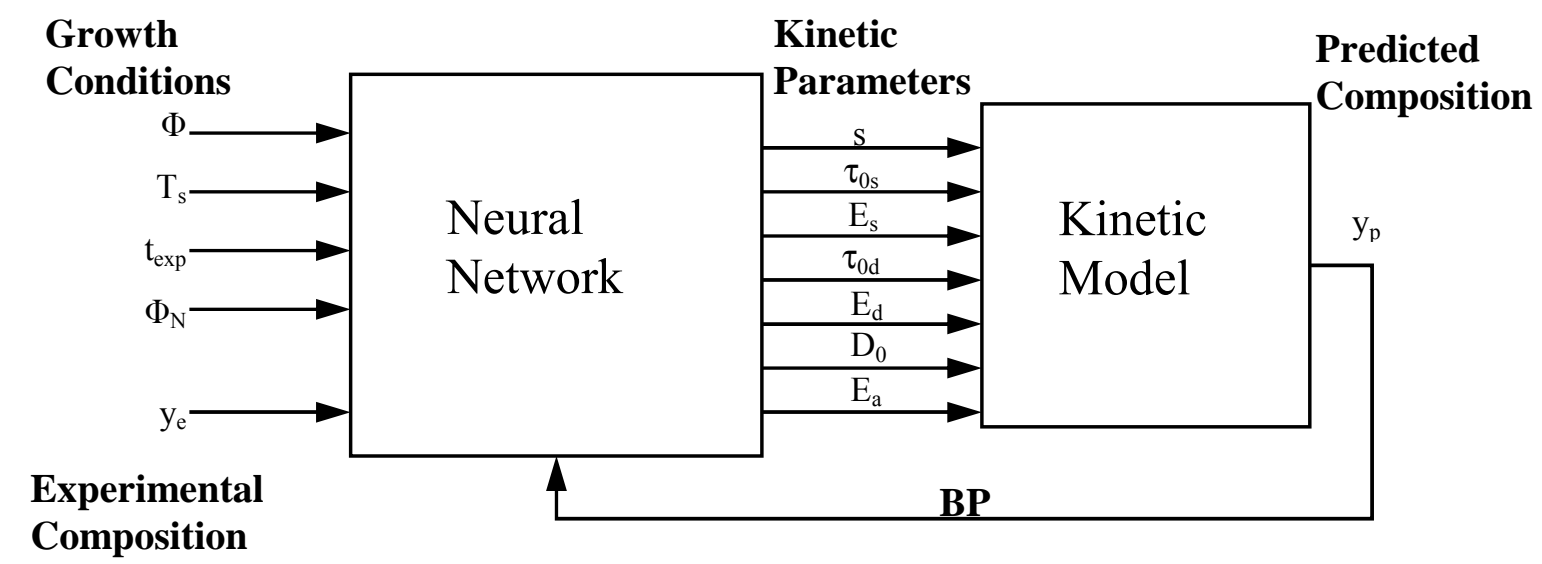

Figure 3.5 The structural design of the hybrid neural network model capable of predicting the composition at the interfaces of mixed anion III-V heterostructures.

The output of the kinetic model component can be used to determine the error of the hybrid neural network. The predicted composition is compared to the experimental composition data, and the mean squared difference between the two vectors determines the network error. This network error can be "back-propagated" through the kinetic model component, essentially by computing the partial derivatives of the kinetic model with respect to each of the process parameters [56]. Using this procedure, the contribution of each kinetic parameter to the hybrid neural network error is obtained, and these are used to calculate an error signal for the neural network component. This network error is then propagated backward through the neural network component, and learning occurs by minimizing the error via modification of the neural network component weights. This procedure is repeated for the set of input/output data until convergence is achieved, at which point the process parameters for the kinetic model are known explicitly and can be used in the first principles kinetic model to predict the composition at the interfaces of mixed anion heterostructures. 


\subsection{Summary}

The effects of anion exchange, segregation, and diffusion at the interfaces of mixed anion heterostructures have been introduced, and the characterization and modeling techniques used for this research have been established. The research efforts described in this chapter provide an essential foundation for constructing a semiempirical model of anion exchange based on hybrid neural networks. The hybrid neural network model developed in this section is implemented in Chapter 6 and provides a greater understanding of the microscopic processes, occurring at the interfaces of mixed anion III-V heterostructures. The compositional profiles present at the interfaces of mixed anion heterostructures is the focus of the next chapter. 


\section{CHAPTER 4}

\section{ANION EXCHANGE - INTERFACE PROFILES}

The determination of the compositional profiles present at the interfaces of mixed anion III-V heterostructures was the initial stage for the analysis of anion exchange performed in this research. This chapter introduces a fundamental investigation of anion exchange for both $\mathrm{P} / \mathrm{As}$ heterostructures and $\mathrm{As} / \mathrm{Sb}$ heterostructures. The results are examined based on the understanding of anion exchange outlined thus far and clearly identify the microscopic processes that lead to the formation of specific interface profiles. The primary material systems used for the analysis of the compositional profiles were the $\mathrm{GaInP} / \mathrm{GaAs}$ system and both the GaAsSb/GaAs and GaSbAs/GaSb system.

\subsection{Ga $\mathrm{In}_{1-\mathrm{x}} \mathrm{P} / \mathrm{GaAs}$ Heterostructures}

There is considerable interest in substituting $\mathrm{Ga}_{\mathrm{x}} \mathrm{In}_{1-\mathrm{x}} \mathrm{P} / \mathrm{GaAs}$ heterostructures for AlGaAs/GaAs. The large band offset and absence of issues associated with the chemical reactivity of aluminum during device processing make the GaInP/GaAs heterostructures an attractive alternative. The $\mathrm{Ga}_{\mathrm{x}} \operatorname{In}_{1-\mathrm{x}} \mathrm{P}$ ternary is lattice matched to GaAs with a composition of $x=0.5$ and has several applications in visible light emitting devices. The lattice constant of GaInP is almost a linear function of the composition, varying between the binary values of $a_{\mathrm{GaP}}=5.4512 \AA$ and $a_{\mathrm{InP}}=5.8686 \AA$. GaInP has a direct to indirect band gap change that occurs at $x=0.74$, which is far away from the GaAs lattice matched composition [57]. The growth of GaInP/GaAs by gas-source MBE has been reported, and the P-for-As exchange reaction was found to occur at the interfaces [58]. While 
several materials related issues are known to occur during growth of $\mathrm{GaInP} / \mathrm{GaAs}$, these heterostructures have been used to design HBTs with superior characteristics compared to $\mathrm{AlGaAs} / \mathrm{GaAs}$ devices $[59,60]$.

\subsubsection{Experimental Design}

The investigation of anion intermixing at the interfaces of $\mathrm{Ga}_{\mathrm{x}} \mathrm{In}_{1-\mathrm{x}} \mathrm{P} / \mathrm{GaAs}$ heterostructures grown by MBE proceeded by implementing a D-optimal experiment consisting of 29 heterointerfaces. The designed experiment for these structures required that the heterointerfaces be grown continuously on a single substrate, forming a 29-period GaInP/GaAs SL. Each period of the SL consists of a $400 \AA$ buffer layer grown under optimal conditions (i.e., GaAs growth at $T_{\mathrm{s}}=600^{\circ} \mathrm{C}, P_{\mathrm{As}_{4}}=6 \times 10^{-6}$ Torr or GaInP growth at $T_{\mathrm{s}}=520^{\circ} \mathrm{C}, P_{\mathrm{P}_{2}}=5 \times 10^{-6}$ Torr with a $2 \times 4$ surface reconstruction) and a heterointerface composed of $50 \AA$ of GaAs and $50 \AA$ of GaInP grown at the conditions specified by the experimental trials with a growth interrupt at the interface. The growth interrupt allows the static surface of the $50 \AA$ GaAs or GaInP layer to be exposed to the dissimilar anion, using a non-growing anion exposure technique. In order to decrease the overall thickness of this structure, the GaInP/GaAs SL was divided into two 9-period SL structures (Sample 1 and Sample 2) and one 11-period SL structure (Sample 3). These structures were grown such that Sample 2 and 3 contain the last two interfaces from the previous sample as their first two heterointerfaces, allowing for duplicate trials and a total of 33 heterointerfaces.

The six input parameters for this statistical experiment included substrate temperature $\left(T_{\mathrm{s}}\right)$, phosphorus flux, arsenic flux, arsenic species $\left(\mathrm{As}_{4}\right.$ or $\left.\mathrm{As}_{2}\right)$, layer growth order (normal or inverted configuration), and anion exposure time ( $\left.t_{\exp }\right)$ at the interface. 
The experimental ranges for each parameter are provided in Table 4.1, and the growth conditions for the designed experiment are provided in Table 4.2. Epitaxial growth was performed on undoped GaAs (001) epi-ready substrates in a Riber 2300 solid-source molecular beam epitaxy (SSMBE) system equipped with a valved As cracker and a threezoned valved P cracker. The surface reconstructions at the interfaces of the SL structures were observed during MBE growth using in-situ RHEED. The structural properties of the GaInP/GaAs SLs were characterized using ex-situ HRTEM.

Table 4.1 The input parameter settings for the anion exchange As/P experimental design.

\begin{tabular}{|l|l|l|l|}
\hline Anion Exchange As/P Experimental Design \\
\hline Parameter Description & Abbreviation & Symbol & Settings \\
\hline Substrate Temperature & $\mathrm{T} \_$Sub & $T_{\mathrm{s}}$ & $475^{\circ} \mathrm{C}$ to $520^{\circ} \mathrm{C}$ \\
\hline Anion Exposure Time & Int_Sk & $t_{\exp }$ & $10 \mathrm{~s}$ to $60 \mathrm{~s}$ \\
\hline Arsenic Species & As_sp & & $\mathrm{As}_{4}\left(600^{\circ} \mathrm{C}\right)$ or $\mathrm{As}_{2}\left(900^{\circ} \mathrm{C}\right)$ \\
\hline As$_{4}$ Flux (BEP) & $\mathrm{As}_{4} \_$Flux & $P_{\mathrm{As}_{4}}$ & $2 \times 10^{-6}$ Torr, $6 \times 10^{-6} \mathrm{Torr}$ \\
\hline $\mathrm{P}_{2}$ Flux (BEP) & $\mathrm{P}_{2}$ Flux & $P_{\mathrm{P}_{2}}$ & $2 \times 10^{-6}$ Torr, $5 \times 10^{-6} \mathrm{Torr}$ \\
\hline Layer Growth Order & Grth_Ord & & Normal or Inverted \\
\hline
\end{tabular}

Table 4.2 The MBE growth conditions for the GaInP/GaAs SL structures.

\begin{tabular}{|l|l|l|}
\hline GaInP/GaAs Growth Conditions \\
\hline Growth Condition & Symbol & Settings \\
\hline Ga Flux (BEP) & $P_{\mathrm{Ga}}$ & $1.5 \times 10^{-7}$ Torr \\
\hline In Flux (BEP) & $P_{\mathrm{In}}$ & $3.0 \times 10^{-7}$ Torr \\
\hline GaAs Growth Rate & $v_{\mathrm{GaAs}}$ & $167 \AA / \mathrm{min}$ \\
\hline GaInP Growth Rate & $v_{\mathrm{GaInP}}$ & $83 \AA / \mathrm{min}$ \\
\hline
\end{tabular}




\subsubsection{HRTEM and RHEED Analysis}

The characterization of anion exchange at the interfaces of $\mathrm{Ga}_{\mathrm{x}} \mathrm{In}_{1-\mathrm{x}} \mathrm{P} / \mathrm{GaAs}$ heterostructures requires the determination of both interface composition and structure. The lattice matched $\mathrm{GaInP} / \mathrm{GaAs}$ heterostructures grown for this experiment were analyzed via HRTEM and shown to exhibit misfit dislocations at several of the interfaces. The [110] cross-section dark field TEM images of the SL structures were formed by the (002) reflection (normal to the interface), which provides a condition where the interface is near the edge-on position, allowing for detailed structural characterization. The (002) type reflection is also extremely sensitive to the chemistry of the structure. The results for Sample 1 are shown in Figure 4.1 where the GaInP layers are imaged as bright bands, the GaAs layers are imaged as dark gray bands, and the growth direction is up.

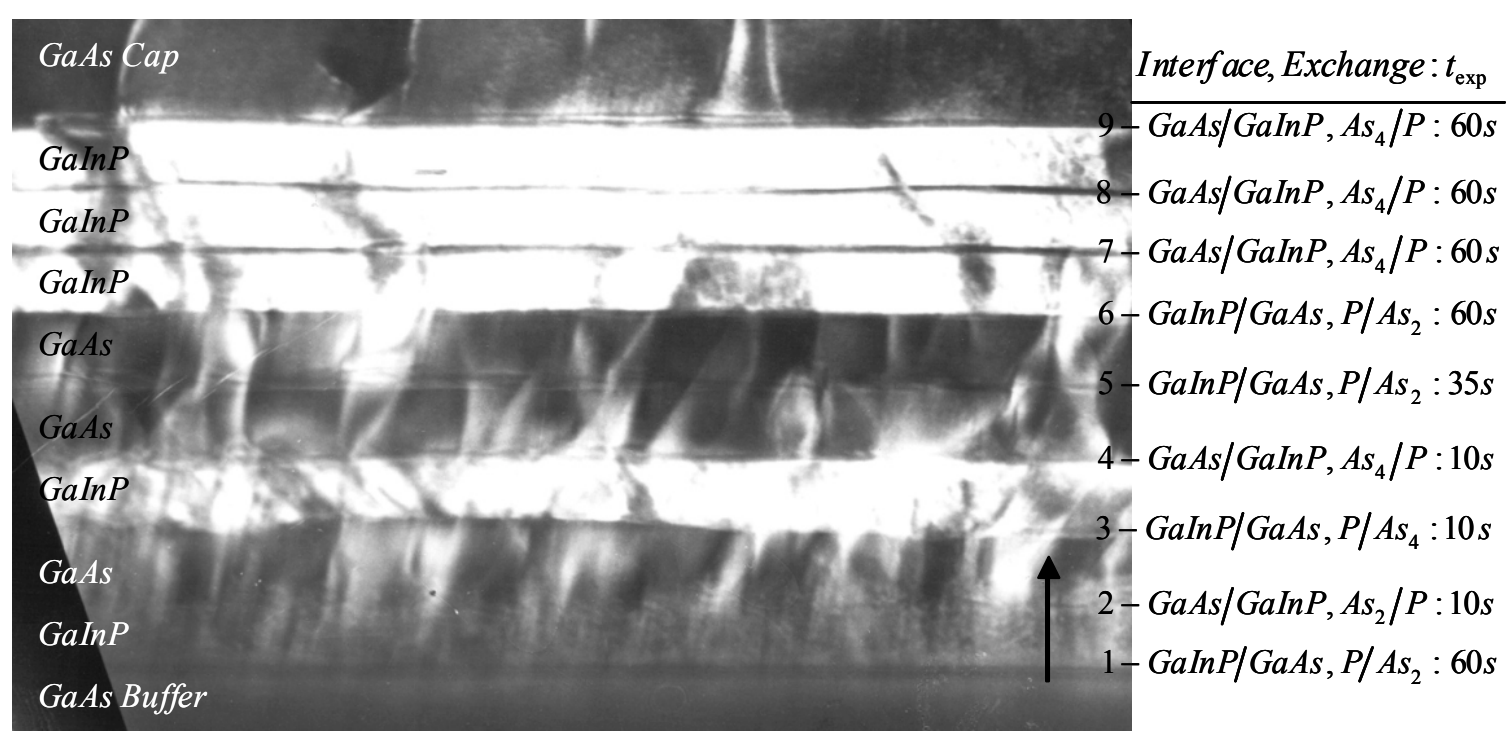

Figure 4.1 The TEM image for Sample 1, the GaInP/GaAs SL structure composed of nine heterointerfaces.

The mixed anion heterointerfaces corresponding to the experimental trials are indicated adjacent to the image, along with the type of exchange (As/P or P/As) and the 
dissimilar anion exposure time. The majority of the heterointerfaces for Sample 1 exhibit relatively smooth interfaces after exposure to the dissimilar anion and subsequent growth. The interfaces that are directly observed as having roughened regions correspond to the $2^{\text {nd }}(2)$ heterointerface $\left(\mathrm{As}_{2}\right.$ exposure of GaInP) and the $3^{\text {rd }}(3)$ heterointerface $\left(\mathrm{P}_{2}\right.$ exposure of $\mathrm{As}_{4}$ grown GaAs). In general, this indicates that the anion exchange reaction occurs to a greater extent for these two anion exposure conditions. The $\mathrm{As}_{2}$ exposure of GaInP appears to result in a graded interface or mixed region, while the $\mathrm{P}_{2}$ exposure of $\mathrm{As}_{4}$ grown GaAs exhibits interfacial roughening, indicating anion replacement and ensuing local strain. The results obtained for Sample 2 are shown in Figure 4.2, and in comparison to the first sample, significantly more misfit dislocations are apparent.

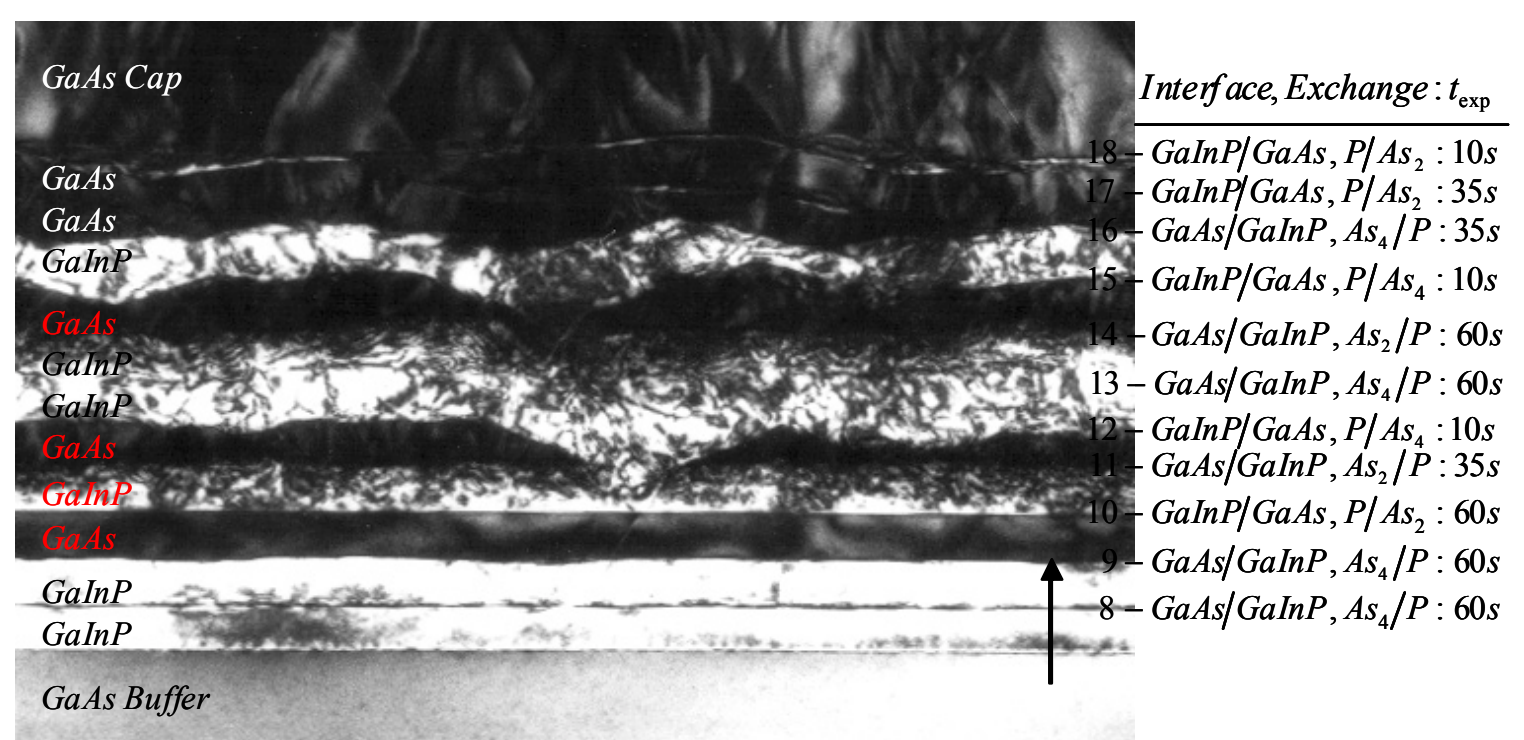

Figure 4.2 The TEM image for Sample 2, the GaInP/GaAs SL structure composed of eleven heterointerfaces.

The initial three heterointerfaces for Sample 2, consisting of $\mathrm{As}_{4}$-exposed GaInP and $\mathrm{P}_{2}$-exposed $\mathrm{As}_{2}$ grown GaAs, have reasonably abrupt interfaces. Conversely, the $4^{\text {th }}$ (11) and $7^{\text {th }}$ (14) heterointerfaces with $\mathrm{As}_{2}$-exposed GaInP exhibit anion intermixing, and 
the $5^{\text {th }}(12)$ and $8^{\text {th }}(15)$ heterointerfaces with $\mathrm{P}_{2}$-exposed $\mathrm{As}_{4}$ grown $\mathrm{GaAs}$ show evidence of extensive interfacial roughening. These results are in agreement with those obtained for Sample 1, indicating that a correlation exists between the constituents of anion exchange and the resulting interface profile. It should be noted that the first $400 \AA$ GaInP layer in Sample 2 is grown lattice-matched on the $2000 \AA \mathrm{As}_{4}$ grown GaAs buffer with an atomically abrupt interface. This non-exposure growth indicates that a sharp $\mathrm{GaInP} / \mathrm{GaAs}$ interface can be obtained when exchange is minimized and that anion exchange is the mechanism driving surface morphology at the interface of mixed anion heterostructures.

The results obtained for the heterointerfaces in Sample 1 and Sample 2 indicate that the $\mathrm{P}_{2} / \mathrm{GaAs}_{4}$ and $\mathrm{As}_{2} / \mathrm{GaInP}$ exchange processes can lead to interface degradation, while the $\mathrm{P}_{2} / \mathrm{GaAs}_{2}$ and $\mathrm{As}_{4} / \mathrm{GaInP}$ exchange processes result in minimal interface roughening. Based on these results, the last three heterointerfaces in Sample 2 are expected to be relatively smooth. This is not the case due to memory effects, i.e., the presence of defects such as interface roughness and dislocations that propagate from the layers below. The increase in dislocations for Sample 2 may be attributed to a substrate temperature controller malfunction. The $400 \AA$ GaAs and GaInP layers for Sample 2 indicated in red were grown while the controller was unable to reach the target substrate temperature, resulting in substrate temperature fluctuations. As noted above, the last two heterointerfaces from Sample 2 are repeated in Sample 3. These heterointerfaces are shown in Figure 4.3, and exhibit abrupt interface structures.

The heterointerfaces observed in Sample 3 support the apparent relationship between anion exchange conditions (i.e., species of the dissimilar anion and type of 
surface) and interface structure discussed thus far. Specifically, we note that the $4^{\text {th }}(20)$, $5^{\text {th }}(21), 9^{\text {th }}(25)$, and $12^{\text {th }}(28)$ heterointerfaces in Sample 3 are $\mathrm{As}_{2} / \mathrm{GaInP}$ exchange and exhibit intermixing. The $6^{\text {th }}(22), 8^{\text {th }}(24)$, and $10^{\text {th }}(26)$ heterointerfaces are $\mathrm{P}_{2} / \mathrm{GaAs}_{4}$ exchange, resulting in interface roughness, and the remaining heterointerfaces are $\mathrm{P}_{2} / \mathrm{GaAs}_{2}$ and $\mathrm{As}_{4} / \mathrm{GaInP}$ exchange with reasonably abrupt interfaces. Although the heterointerfaces in Sample 3 have intermixing and roughness present for more than half of the interfaces along with memory effects, the effects of anion exchange on the interface profiles are still distinct.

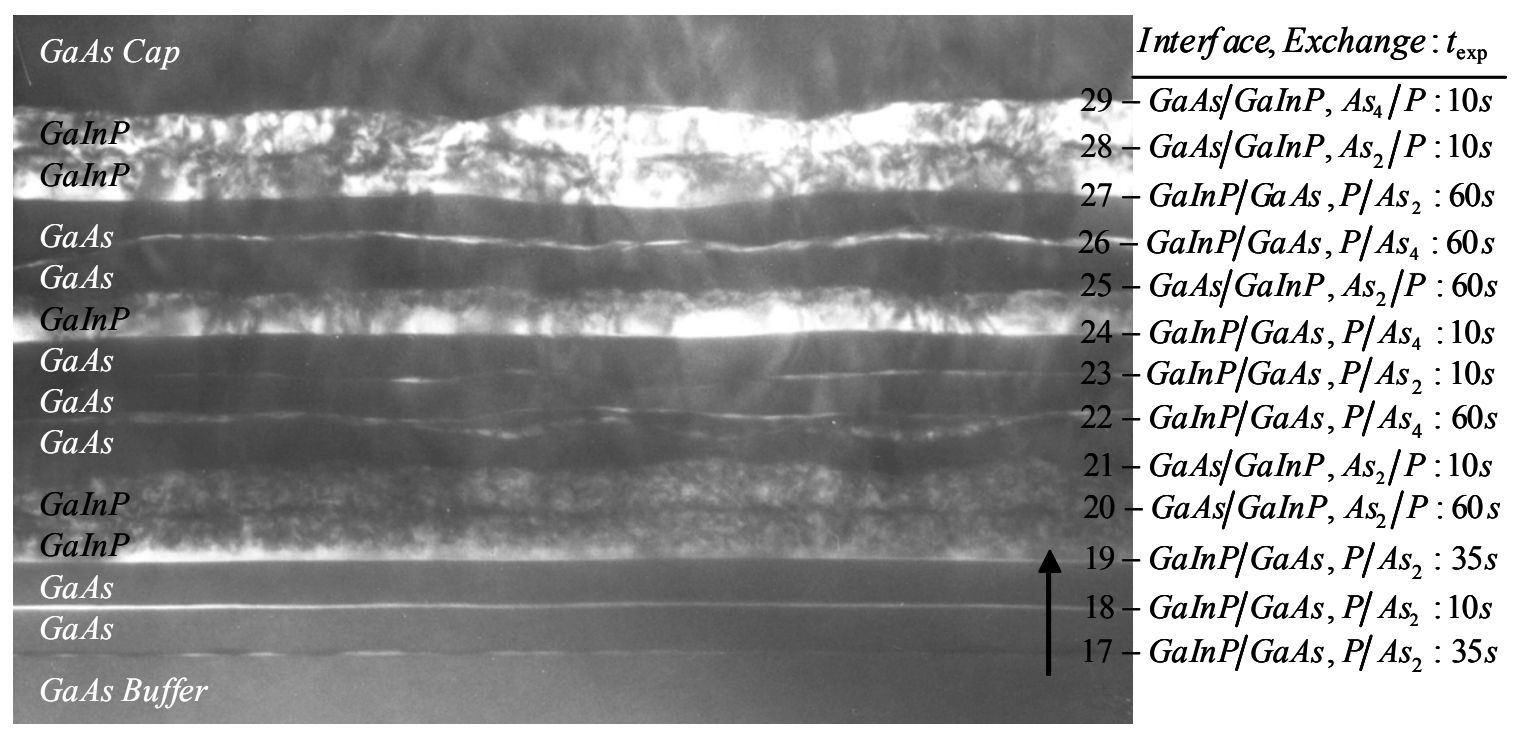

Figure 4.3 The TEM image for Sample 3, the GaInP/GaAs SL structure composed of thirteen heterointerfaces.

The surface reconstructions present during $\mathrm{MBE}$ growth of the $\mathrm{GaInP} / \mathrm{GaAs} \mathrm{SL}$ structures were monitored in-situ via RHEED. The results obtained from RHEED observations in the [011] direction confirm the interface morphology observed by HRTEM. Specifically, for the heterointerfaces with minimal interface roughness, a $2 \times$ RHEED pattern was observed during anion exchange, corresponding to a relatively 
smooth surface. Conversely, the heterointerfaces with interface roughness exhibited both a spotty $2 \times$ and a disordered $1 \times$ RHEED pattern when exposed to the dissimilar anion, indicating surface nucleation and the formation of 3-D islands on the surface.

\subsubsection{Discussion}

The microscopic processes occurring at the interfaces of the GaInP/GaAs SL structures can be identified on the basis of the interface profiles present. The heterointerfaces formed by the $\mathrm{As}_{4}$ exposure of $\mathrm{GaInP}$ and the $\mathrm{P}_{2}$ exposure of $\mathrm{As}_{2}$ grown

GaAs exhibit an exchange process that occurs over the first few monolayers without considerable strain. In contrast, the exchange process for the $\mathrm{P}_{2}$ exposure of $\mathrm{As}_{4}$ grown GaAs increases the strain at the surface during growth, and the interface roughening observed is likely due to a 2-D to 3-D surface transition. A similar exchange process occurs for the $\mathrm{As}_{2}$ exposure of GaInP, with the distinction that the surface strain is decreased by means of a diffusion mechanism. There is significant evidence that the dissimilar anon species and surface structure are the primary factors contributing to anion intermixing. There exist secondary effects associated with changes in substrate temperature, group-V pressures, and dissimilar anion exposure time; however, only small variations such as increased roughening for longer exposures have been observed. The detailed experimental conditions for each heterointerface, along with qualitative descriptions of the HRTEM images, and a summary of the RHEED pattern data are provided in Appendix B.

In order to fully investigate the effects of anion exchange during the MBE growth of GaInP/GaAs heterointerfaces, cross-sectional TEM was used to study the material defects present in these structures. The microstructure analysis performed by Wang et al. 
indicated that special misfit dislocation configurations, known as dislocation dipoles, were present at the $\mathrm{GaInP} / \mathrm{GaAs}$ interface [61]. These dislocations are generated during epitaxial growth, but are not produced by conventional lattice mismatch. They are likely the result of the exchange process and subsequent 3-D island formation occurring for the $\mathrm{P}_{2} / \mathrm{GaAs}_{4}$ heterointerfaces. Dislocations were also observed inside the GaInP layers with threading dislocations increasing in density with the number of epilayers. These dislocations were determined to be coincident with the presence of lateral compositional modulation in the GaInP layers [62]. The relationship between the microscopic processes occurring during growth and the observed microstructures is difficult to determine. However, it is well known that compositional modulation is caused by processes on the growing surface rather than in the bulk. Therefore, it is reasonable to conclude that compositional modulation arises from strain-induced surface roughening due to anion exchange at the interfaces of $\mathrm{GaInP} / \mathrm{GaAs}$ heterostructures.

\section{2 $\mathrm{GaAs}_{1-\mathrm{y}} \mathrm{Sb}_{\mathrm{y}} / \mathrm{GaAs}$ and $\mathrm{GaSb}_{1-\mathrm{y}} \mathrm{As}_{\mathrm{y}} / \mathrm{GaSb}$ Heterostructures}

The $\mathrm{GaAs}_{1-\mathrm{y}} \mathrm{Sb}_{\mathrm{y}} / \mathrm{GaAs}$ heterostructure system has obtained considerable attention for its potential in optical devices. Light emitting diodes, laser diodes, and vertical cavity surface emitting lasers based on the GaAsSb material system have been achieved $[63,64,65]$. The material system also offers the opportunity to investigate the electronic and optical properties in a staggered band alignment heterojunction [66,67]. While progress has been made experimentally in identifying band alignments, investigations of anion incorporation for MBE grown GaAsSb indicate that the exchange process makes composition control in this material system difficult [68]. 


\subsubsection{Experimental Design}

The investigation of anion intermixing at the interfaces of $\mathrm{As} / \mathrm{Sb}$ heterostructures proceeded by constructing two D-optimal experiments, one for each type of heterointerface, and growing test structures at the endpoints of the experimental parameter space. These experiments focused on the characterization of 20-period $\mathrm{GaAs}_{1-}$ ${ }_{\mathrm{y}} \mathrm{Sb}_{\mathrm{y}} / \mathrm{GaAs}$ and $\mathrm{GaSb}_{1-\mathrm{y}} \mathrm{As}_{\mathrm{y}} / \mathrm{GaSb}$ SLS structures. Conditions such as substrate temperature and anion exposure time were varied to impact the degree of anion intermixing. Epitaxial growth was performed on undoped GaAs (001) and GaSb (001) epi-ready substrates in a Varian Gen-II solid-source molecular beam epitaxy system equipped with both As and Sb valved crackers. RHEED was used to observe the surface reconstructions during $\mathrm{Sb}_{2}$ exposure of $\mathrm{GaAs}$ and $\mathrm{As}_{4}$ exposure of $\mathrm{GaSb}$. Growth rates were determined in-situ by RHEED oscillations and set to be $0.5 \mathrm{ML} / \mathrm{s}$ for GaAs and 0.8 $\mathrm{ML} / \mathrm{s}$ for GaSb. Structural properties were measured by high-resolution x-ray diffraction, using $\mathrm{Cu} K \alpha$ radiation to obtain $\theta / 2 \theta$ linescans for all samples. Dynamical simulations were conducted for samples that exhibited anion exchange (i.e., superlattice formation). HRXRD simulation and analysis proceeded by using the Philips High Resolution Epitaxy simulation software to determine the composition at the SLS interfaces.

\subsubsection{HRXRD and Simulation}

HRXRD was used to characterize and compare the exchange process at the interfaces of the $\mathrm{As} / \mathrm{Sb}$ heterostructures. Superlattices are often characterized nondestructively using HRXRD. Dynamical simulation of diffraction peaks can provide accurate approximations of layer structures, compositions, and strain profiles. Investigations performed by Wang et al. [69] have indicated that anion intermixing in 
$\mathrm{As} / \mathrm{Sb}$ heterostructures occurs due to either $\mathrm{Sb}$ segregation into As-based overlayers or As incorporation into Sb-based underlayers. The SLS structures designed for this research have been characterized using this fundamental behavior as the basis for simulation and analysis of the anion exposed surfaces.

The initial approach for simulating the $\mathrm{As} / \mathrm{Sb}$ heterostructures consisted of modeling the basic anion intermixing present within the superlattice based on growth mechanisms. In-plane d-spacing measurements obtained from RHEED horizontal linescans indicate that the intermixed layers are commensurate with the substrate for both $\mathrm{Sb}_{2}$-exposed GaAs and $\mathrm{As}_{4}$-exposed GaSb material. These SLS layers, which are formed as a result of anion exposure, consist of coherently strained GaAsSb and are modeled as such. Two-dimensional equivalent alloy compositions and SL period thickness were determined from the zero-order SL peak position and satellite peak positions respectively. Each superlattice interface was initially modeled as consisting completely of exchanged layers (i.e., a uniform intermixed layer of one or more MLs of material). Figure 4.4 shows the basic simulation structures for an $\mathrm{As}_{4}$-exposed GaSb SLS and an $\mathrm{Sb}_{2}$-exposed GaAs SLS. This is the simplest model for anion intermixing, and provides insight into the average incorporation of the impinging anion. While these simulations do not predict SL peak intensities well, they provide exact agreement with experimental SL peak positions and serve as reference spectra during simulation structure optimization.

After the SL peak positions are confirmed, determination of the SL peak intensities requires a more complex approach. Analysis performed by Huang et al. [70] examined the sensitivity of SL peak intensities for various structural parameters. The analysis showed that changes in interface composition resulted in only small variations in 
SL satellite peak intensities for layers of constant thickness. They determined that the higher-order SL satellites were much more sensitive to the relative thickness of the modeled layers. This indicates that the structure of each SL layer, consisting of a layer thickness and compositional profile, is the most important factor in determining SL peak intensity.

(a) $\mathbf{G 3 2 0}-\mathrm{As}_{4} / \mathbf{G a S b}$

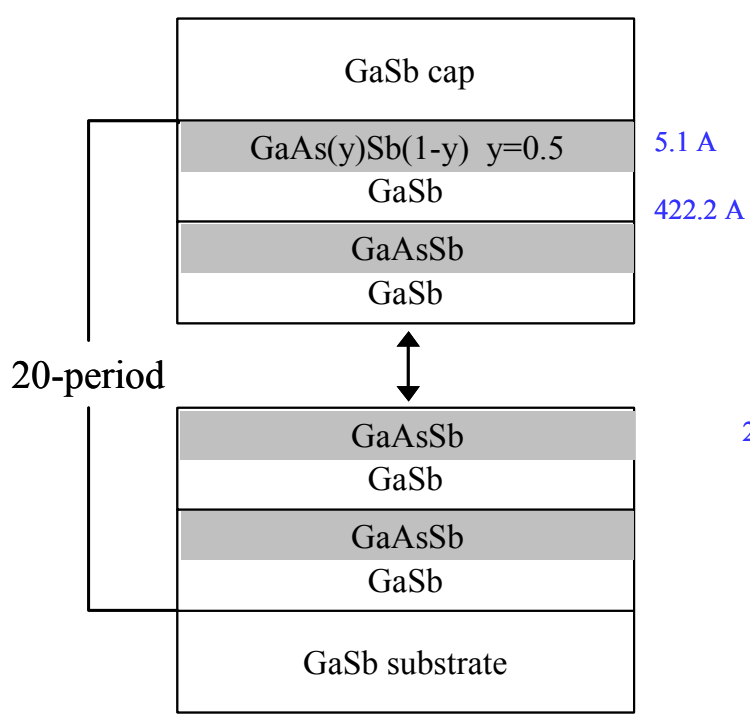

(b) $\mathbf{G 1 7 1}-\mathrm{Sb}_{2} / \mathrm{GaAs}$

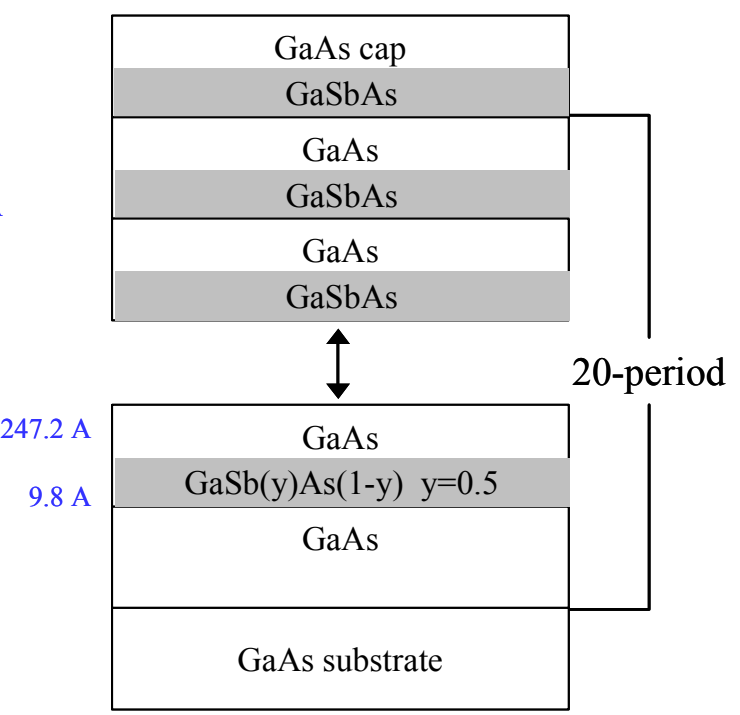

Figure 4.4 The initial HRXRD simulation structures representative of (a) $\mathrm{As}_{4}$-exposed $\mathrm{GaSb}$ superlattices and (b) $\mathrm{Sb}_{2}$-exposed GaAs superlattices.

In order to obtain exact SL peak intensity fitting, one must systematically iterate through combinations of SL layer structures. In the case of the As/Sb material system, the prevalent structures consist of the compositional interface profiles produced by anion exchange and segregation. Optimization of these simulated structures can be achieved using an iterative approach along with the following criteria. First, the thickness of each monolayer of the simulated structure must be equivalent to the calculated pseudomorphic thickness for one monolayer at the modeled composition. Second, the compositions of 
the modeled layers must be chosen such that the zero-order SL peak position relative to the substrate is maintained. Finally, the simulation must predict the order of the SL satellite peak intensities, meaning that it must correctly resolve which peak has the largest intensity, which is second largest, and so forth.

Simulations of $\mathrm{As}_{4}$-exposed $\mathrm{GaSb}$ material required modeling of exchanged layers only, while simulations of $\mathrm{Sb}_{2}$-exposed GaAs material required modeling of both exchanged layers and layers containing segregation (i.e., an exponentially decaying compositional profile toward the sample surface). Modeling of exchanged layers required simulating $\mathrm{GaSb}_{1-\mathrm{y}} \mathrm{As}_{\mathrm{y}}$ layers with constant composition $y$, and increasing the thickness of these layers a monolayer at a time until the correct SL peak intensities were obtained. Segregation modeling was accomplished using $\mathrm{GaAs}_{1-\mathrm{y}} \mathrm{Sb}_{\mathrm{y}}$ layers with exponential composition variations $\left(y=e^{-t}\right)$ at the interfaces, where $t$ is the layer thickness in the growth direction. Figure 4.5 shows the optimized simulation structures for an $\mathrm{As}_{4}$-exposed $\mathrm{GaSb}$ SLS and a $\mathrm{Sb}_{2}$-exposed GaAs SLS. The x-ray simulations showed a high degree of sensitivity to both the thickness of the segregated layers and the number of exchanged monolayers. The segregation length primarily affected the intensity of the negative-order satellite peaks with larger segregation lengths resulting in lower intensities than the experimental data [71]. The number of exchanged monolayers affected the positive-order satellite peaks with excess exchanged MLs resulting in lower intensities than measured. 
(a) $\mathbf{G 3 2 0}-\mathrm{As}_{4} / \mathrm{GaSb}$

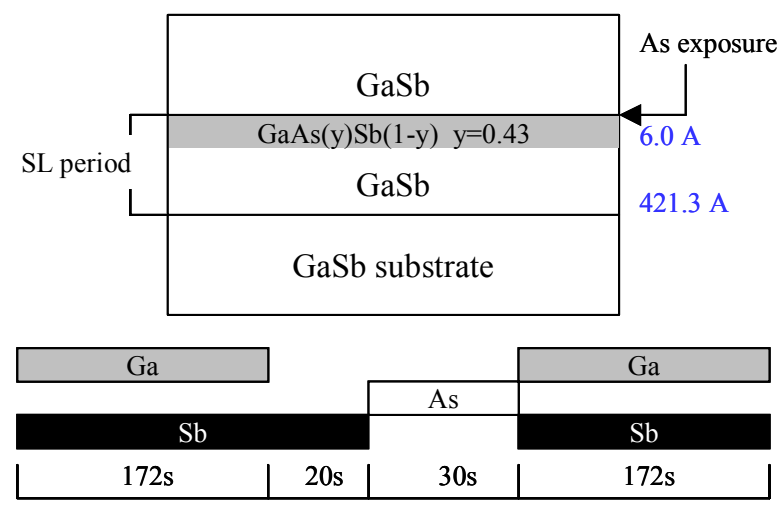

As exposure of $\mathrm{Sb}$-stabilized $\mathrm{GaSb}$

\section{(b) $\mathbf{G 1 7 1}-\mathrm{Sb}_{2} / \mathrm{GaAs}$}

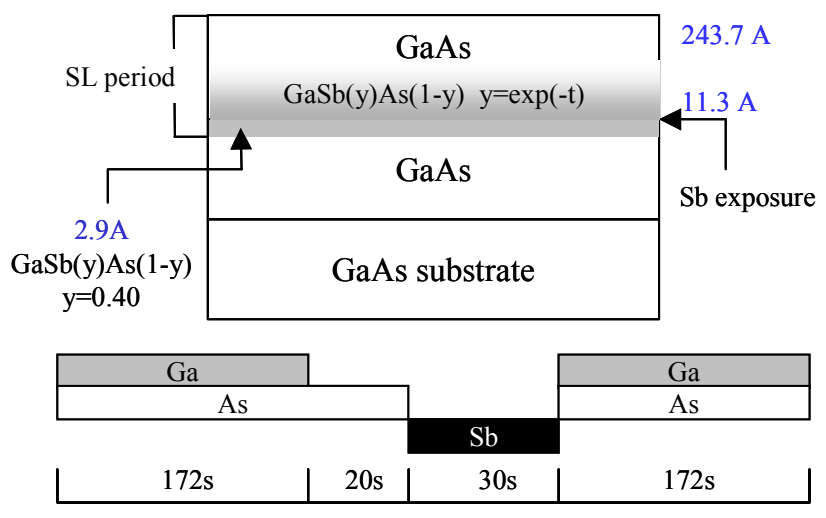

$\mathrm{Sb}$ exposure of As-stabilized GaAs

Figure 4.5 The optimized simulation structures representative of (a) As 4 -exposed GaSb superlattices and (b) $\mathrm{Sb}_{2}$-exposed GaAs superlattices. The simulation structure and corresponding shutter sequence is provided for the first period of each superlattice. 
The goodness-of-fit for each HRXRD simulation was quantified using the root mean squared error (RMSE) of the $\mathrm{x}$-ray spectrum. The RMSE values have been expressed as a percentage of the maximum x-ray intensity for each superlattice. These RMSE values are less than $4 \%$ for $\mathrm{As}_{4}$-exposed GaSb SLS and less than $5 \%$ for $\mathrm{Sb}_{2}$ exposed GaAs SLS, indicating considerable agreement between simulated and experimental x-ray spectra. Excellent reproducibility was obtained for the overall analysis technique. Samples $*$ G405 and $*$ G406 are duplicate $\mathrm{Sb}_{2}$-exposed GaAs experiments for samples G172 and G173 respectively. These GaAsSb/GaAs SLS maintain consistent simulation structures with previous samples and RMSE values of less than $8 \%$ of the maximum x-ray intensity. Similar reproducibility has been obtained for $\mathrm{As}_{4}$-exposed $\mathrm{GaSb}$ superlattices.

\subsubsection{Results and Discussion}

The analysis of Sb-for-As exchange on GaAs was performed utilizing a set of 20period $\mathrm{GaAs}_{1-\mathrm{y}} \mathrm{Sb}_{\mathrm{y}} / \mathrm{GaAs}$ superlattices. Antimony incorporation in the SLS was achieved by the $\mathrm{Sb}_{2}$ exposure of an As-stabilized GaAs surface and an immediate overgrowth of GaAs. The substrate temperature $\left(T_{\mathrm{s}}\right)$ for these samples ranged from $330^{\circ} \mathrm{C}$ to $590^{\circ} \mathrm{C}$ and pressures of $P_{\mathrm{As}_{4}}=2 \times 10^{-6}$ Torr and $P_{\mathrm{Sb}_{2}}=4 \times 10^{-6}$ Torr were used. Sb exposure time $\left(t_{\mathrm{Sb}}\right)$ ranged from $30 \mathrm{~s}$ to $100 \mathrm{~s}$. HRXRD measurements revealed well-defined superlattices with at least five positive-order and five negative-order satellite peaks for samples in the $420^{\circ} \mathrm{C}$ to $550^{\circ} \mathrm{C}$ temperature range. Characteristic x-ray spectra for $\mathrm{Sb}_{2}$-exposed $\mathrm{GaAs}$ SLS and their corresponding simulations are shown in Figure 4.6. HRXRD simulations indicate that these samples consist of a $1.0 \mathrm{ML} \mathrm{GaAs}_{1-\mathrm{y}} \mathrm{Sb}_{\mathrm{y}}$ exchange layer with an average $\mathrm{Sb}$ composition $\left(y_{\mathrm{Sb}}\right)$ of less than 0.50, and 3-5 MLs of Sb segregation with an 
exponentially decaying composition $\left(y_{\mathrm{b} \mathrm{sb}}-y_{\mathrm{t}} \mathrm{sb}\right)$ of 0.40 to 0.10 from layer bottom to top. The small contribution of exchange to the composition profile of these samples is likely due to the dominant effects of the $\mathrm{Sb}$ segregation process at the interfaces.

GaSbAs/GaAs 20-period SL $\left(\mathrm{Sb}_{2}\right)$

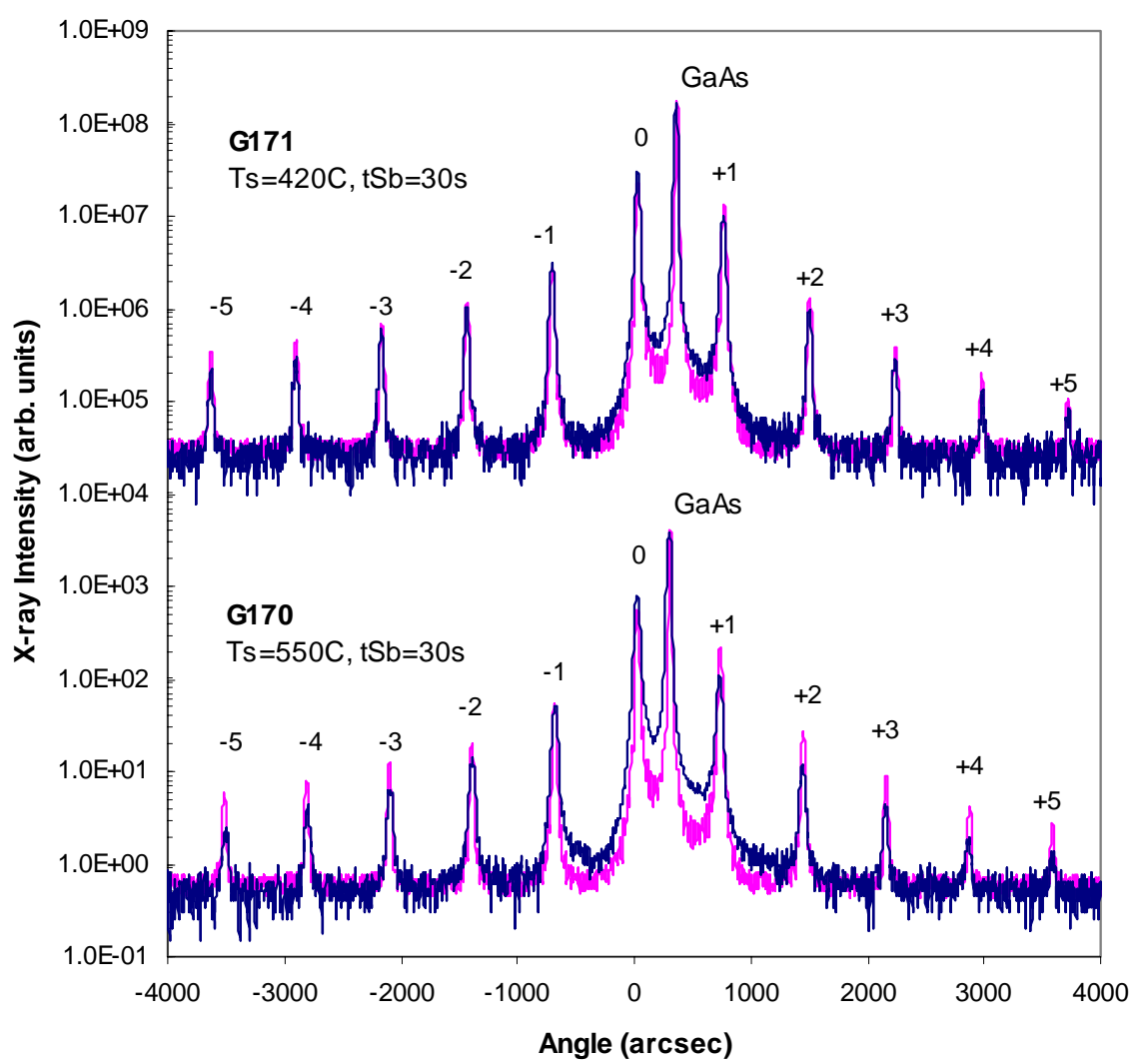

Figure 4.6 The HRXRD $\theta / 2 \theta$ linescans (blue) and simulations (red) for superlattices formed by the $\mathrm{Sb}_{2}$ exposure of As-stabilized GaAs surfaces and immediate overgrowth of GaAs during MBE growth.

Upon examination of the contributions of both anion exchange and segregation relative to exposure time for the $\mathrm{Sb}_{2}$-exposed $\mathrm{GaAs}$ structures, we observed several interesting relationships between growth conditions and $\mathrm{Sb}$ composition at the interface. It is evident that the total $\mathrm{Sb}$ incorporation for a range of short exposures is virtually 
equivalent. We obtained similar average Sb compositions for several short exposure superlattices. This indicates that the surface population of $\mathrm{Sb}$ does not increase for short exposures. Superlattices with exposure times of less than 30s show only small increases in $\mathrm{Sb}$ composition. As expected, $\mathrm{Sb}$ desorption increases with temperature causing a net decrease in $\mathrm{Sb}$ composition.

A set of 20-period $\mathrm{GaSb}_{1-\mathrm{y}} \mathrm{As}_{\mathrm{y}} / \mathrm{GaSb}$ superlattices was grown for the analysis of As-for-Sb exchange on GaSb. Each superlattice period was formed by the $\mathrm{As}_{4}$ exposure of a Sb-stabilized GaSb surface and an immediate overgrowth of GaSb. The substrate temperature $\left(T_{\mathrm{s}}\right)$ for the set of samples was varied from $400^{\circ} \mathrm{C}$ to $500^{\circ} \mathrm{C}$ and pressures of $P_{\mathrm{As}_{4}}=4 \times 10^{-6}$ Torr and $P_{\mathrm{Sb}_{2}}=2 \times 10^{-6}$ Torr were used. The As exposure time $\left(t_{\mathrm{As}}\right)$ ranged from 10s to 30s. HRXRD measurements revealed well-defined superlattices with at least eight positive-order and eight negative-order satellite peaks for samples in the $420^{\circ} \mathrm{C}$ to $450^{\circ} \mathrm{C}$ temperature range. Characteristic $\mathrm{x}$-ray spectra for $\mathrm{As}_{4}$-exposed GaSb SLS and their corresponding simulations are shown in Figure 4.7. Best-fit simulations for samples exposed with As for $10 \mathrm{~s}$ consist of a $1.0 \mathrm{ML} \mathrm{GaSb}_{1-\mathrm{y}} \mathrm{As}_{\mathrm{y}}$ exchange layer with As composition $\left(y_{\mathrm{As}}\right)$ ranging from 0.33 to 0.47 . Simulation and analysis of the $30 \mathrm{~s}$ Asexposed samples revealed a 2.0 ML GaSb $b_{1-y} A s_{y}$ exchange layer with an average As composition $\left(y_{\mathrm{As}}\right)$ of 0.45 . Best-fit simulations were obtained by distributing the As composition such that the first monolayer contained the majority of the exchange. This suggests that the first layer of $\mathrm{Sb}$ atoms depletes as the As-for-Sb exchange reaction proceeds; and a self-limiting As-rich layer forms, hindering further exchange [23]. 


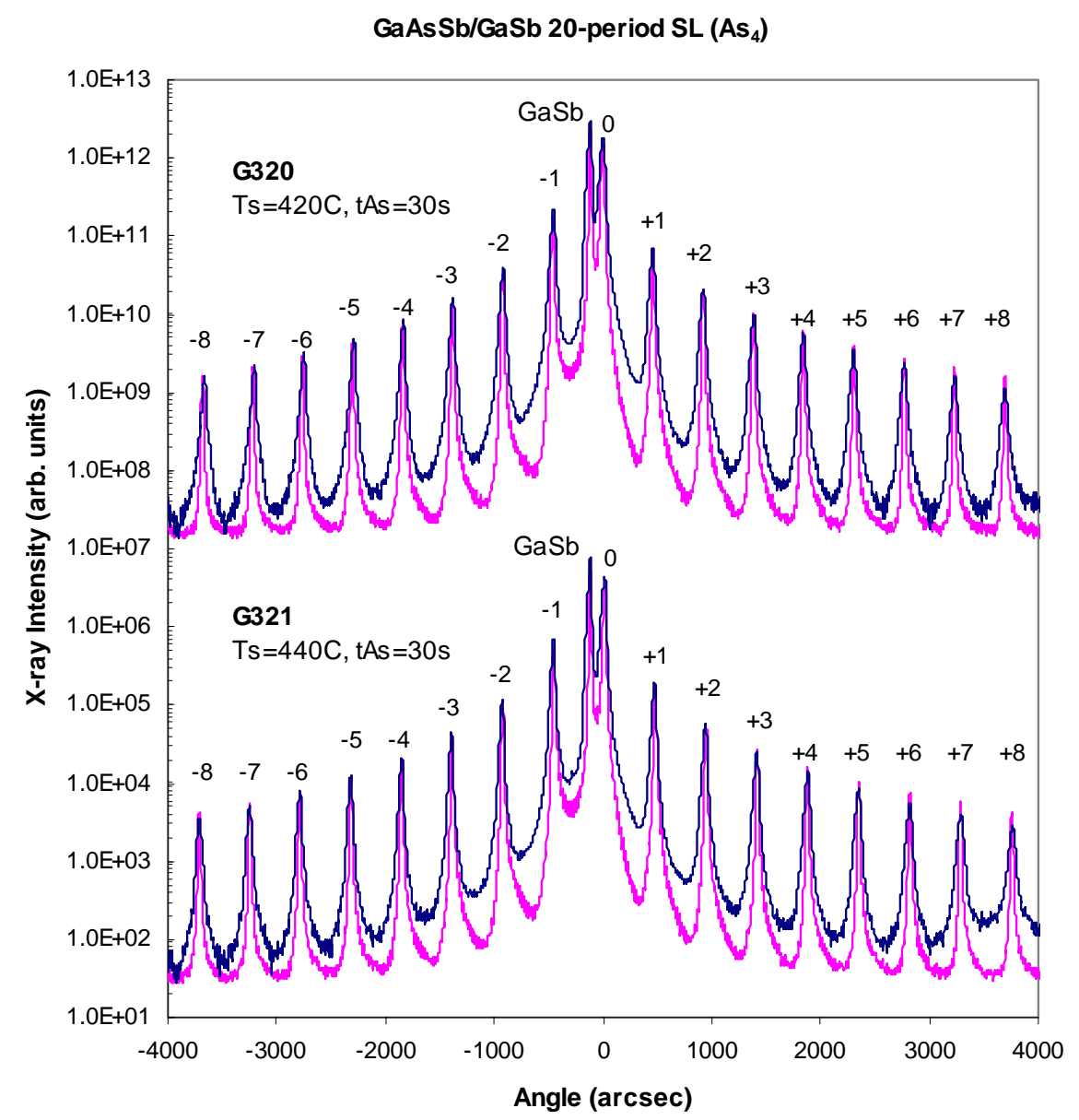

Figure 4.7 The HRXRD $\theta / 2 \theta$ linescans (blue) and simulations (red) for superlattices formed by the $\mathrm{As}_{4}$ exposure of $\mathrm{Sb}$-stabilized $\mathrm{GaSb}$ surfaces and immediate overgrowth of $\mathrm{GaSb}$ during MBE growth.

The simulation results obtained for the $\mathrm{As}_{4}$-exposed GaSb SLS indicate an unexpectedly low total composition of exchanged anions. Table 4.3 contains a summary of the simulation data obtained for several $\mathrm{Sb}_{2}$-exposed GaAs SLS. The simulation data for the $\mathrm{As}_{4}$-exposed GaSb SLS is provided in Table 4.4. There are several comparisons of significant interest that exist with respect to the simulation results. First, the primary process that contributes to anion intermixing for $\mathrm{As}_{4}$-exposed $\mathrm{GaSb}$ is anion exchange. 
This is not the case for $\mathrm{Sb}_{2}$-exposed GaAs for which surface segregation dominates. Second, both anion exposed surfaces exhibit a range of short exposures for which total exchanged anion remains essentially the same for a set temperature. This was observed in $\mathrm{Sb}_{2}$-exposed $\mathrm{GaAs}$ for exposures of $5 \mathrm{~s}$ to $30 \mathrm{~s}$, and in $\mathrm{As}_{4}$-exposed $\mathrm{GaSb}$ for exposures of less than 10s. Third, the $\mathrm{Sb}_{2}$-exposed GaAs superlattices have a larger incorporation of $\mathrm{Sb}$ than the $\mathrm{As}_{4}$-exposed GaSb SLS have As incorporated. One would not expect this to be the case based on bond formation energies [72]. In order to determine if the low As incorporation is related to the use of $\mathrm{As}_{4}$, an $\mathrm{As}_{2}$-exposed GaSb SLS was grown.

The $\mathrm{x}$-ray diffraction spectrum for an $\mathrm{As}_{2}$-exposed GaSb SLS is provided in Figure 4.8. This SLS shares the same growth conditions as the As 4 -exposed GaSb SLS sample G320. These $\mathrm{x}$-ray spectra indicate that $\mathrm{As}_{4}$ is less reactive than $\mathrm{As}_{2}$ to $\mathrm{GaSb}$ surfaces, resulting in a lower As incorporation into GaSb layers. The simulation results corresponding to $\mathrm{Sb}$-for-As exchange on GaAs and As-for-Sb exchange on GaSb provide fundamental insight into the characteristics of the anion exchange process. Both material systems exhibited an anion exchange process with limited contribution to compositional interface profiles relative to the increased exposure times. This indicates that anion exchange can be limited by secondary microscopic processes and may exhibit selflimiting characteristics in some material systems. The details of the characterization work performed for the $\mathrm{As} / \mathrm{Sb}$ heterostructures may be found in reference [73]. 


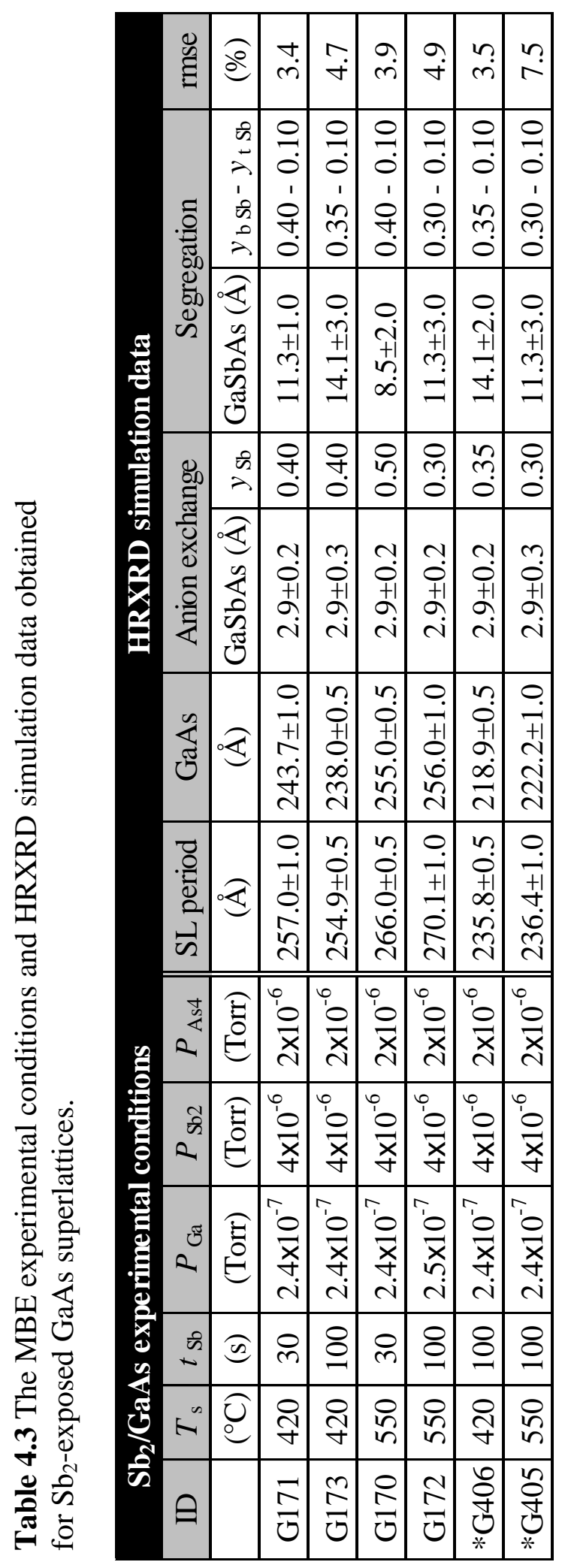




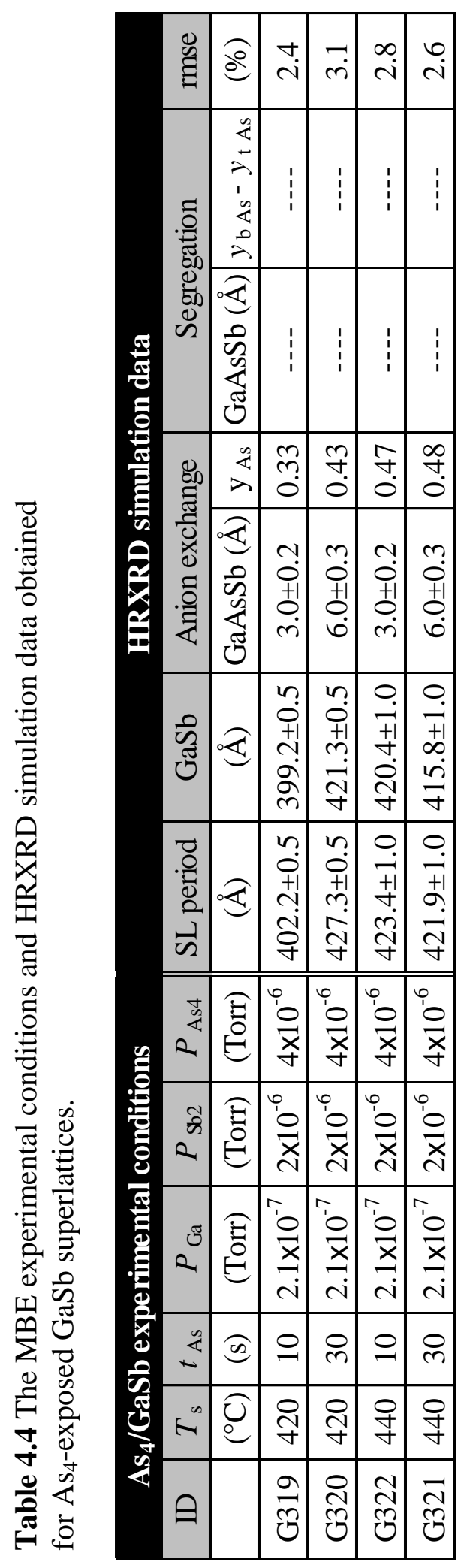




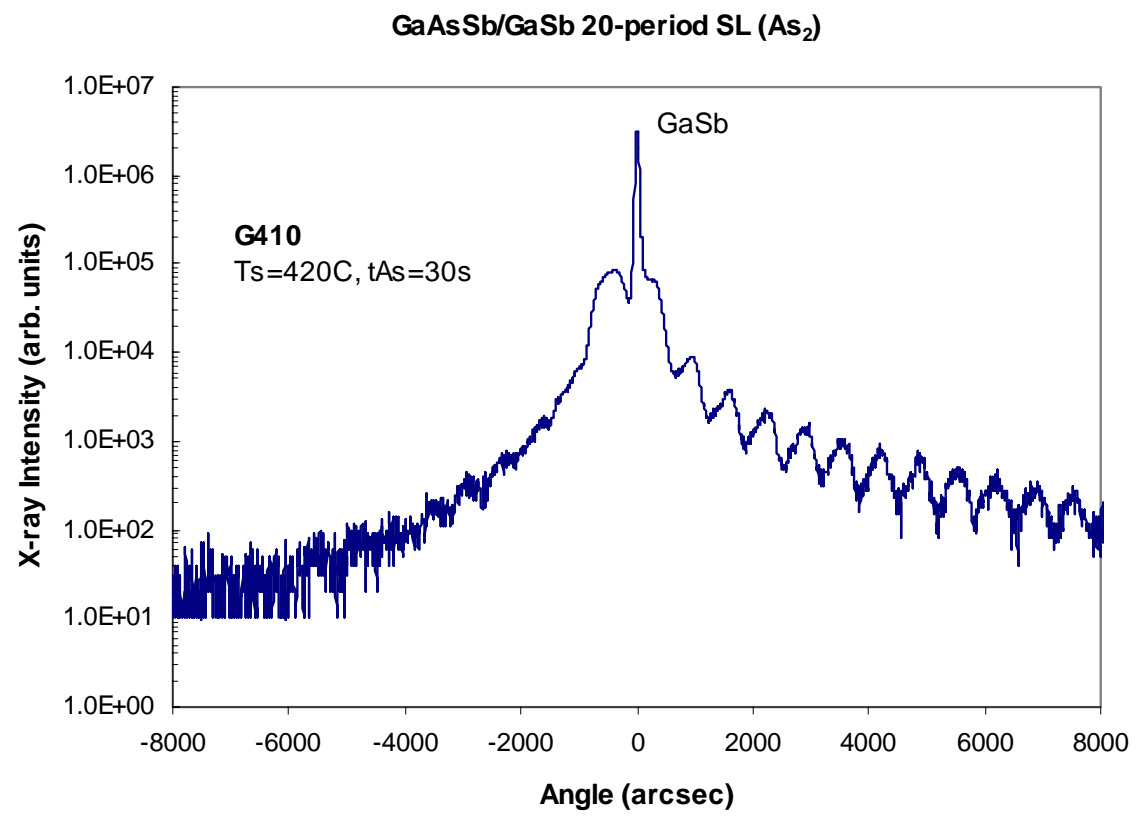

Figure 4.8 The HRXRD $\theta / 2 \theta$ scan for a superlattice formed by the $\mathrm{As}_{2}$ exposure of Sbstabilized GaSb surfaces and immediate overgrowth of GaSb during MBE growth. 


\subsection{Summary}

The compositional interface profiles present for the MBE growth of GaInP/GaAs heterostructures have been determined and correlated with specific anion exchange processes. These structures were examined based on the surface processes contributing to anion intermixing and the formation of structural defects. The $\mathrm{P}_{2} / \mathrm{GaAs}_{4}$ and $\mathrm{As}_{2} / \mathrm{GaInP}$ exchange processes were found to result in considerable interface degradation. The compositional interface profiles for the $\mathrm{GaAsSb} / \mathrm{GaAs}$ and GaSbAs/GaSb structures were determined using HRXRD dynamical simulations. The primary surface process contributing to anion intermixing for $\mathrm{As}_{4}$-exposed $\mathrm{GaSb}$ was determined to be anion exchange, while surface segregation dominated the anion intermixing for $\mathrm{Sb}_{2}$-exposed GaAs. The relationship between atomic surface structure and anion exchange at the interfaces of mixed anion heterostructures is investigated in the next chapter. 


\section{CHAPTER 5}

\section{ANION EXCHANGE - ATOMIC SURFACE STRUCTURE}

The research performed for determining the compositional profiles at the interfaces of mixed anion heterostructures revealed several observations of significance. Specifically, the anion exchange process generates the initial strain energy in the mixed anion layer, and strain accommodation via tetragonal lattice distortion or the introduction of misfit dislocations may occur. The anion exchange process was also determined to be more reactive for specific surfaces, resulting in greater anion intermixing, for which the effects of temperature and anion exposure time were minimal. This chapter provides an investigation of the relationship between anion incorporation and elastic strain energy for the As/P heterostructures. The dependence of anion exchange on substrate temperature and anion exposure time is quantified, and the role of atomic surface structure in the formation of interfaces for mixed anion III-V heterostructures is established. The $\mathrm{GaAsP} / \mathrm{GaAs}$ heterostructure system was used for this analysis of anion exchange.

\subsection{GaAs 1-y $_{\text {y }} / G^{G A A s}$ Heterostructures}

$\mathrm{GaAs}_{1-\mathrm{y}} \mathrm{P}_{\mathrm{y}} / \mathrm{GaAs}$ strained layer multi-quantum wells and superlattices are of great interest for optoelectronic applications. The band gap of $\mathrm{GaAs}_{1-\mathrm{y}} \mathrm{P}_{\mathrm{y}}$ varies almost linearly with composition up to the composition $y=0.45$ and is suitable for both visible and IR emitters and detectors operated between 0.7 and $2.0 \mu \mathrm{m}$. GaAsP is a direct band gap semiconductor for phosphorus compositions ranging from GaAs to $\mathrm{GaAs}_{0.55} \mathrm{P}_{0.45}$ and is indirect from this composition to GaP. The most common alloy composition used in red 
LEDs is $y=0.4$, for which the band gap is direct. The lattice constant of $\mathrm{GaAs}_{1-\mathrm{y}} \mathrm{P}_{\mathrm{y}}$ varies between the binary values of $a_{\mathrm{GaAs}}=5.6533 \AA$ and $a_{\mathrm{GaP}}=5.4512 \AA$. GaAsP layers are under biaxial tension on GaAs (001) substrates, and the critical thickness for epitaxial growth has been determined to be $430 \mathrm{~nm}$ for $y=0.05$ [74]. Investigations of As and $\mathrm{P}$ incorporation in $\mathrm{GaAsP} / \mathrm{GaAs}$ strained layer superlattices have determined that group- $\mathrm{V}$ incorporation characteristics strongly deviate from the linear relationships predicted by Vegard's law [75]. It should be noted that a growth model for the MBE growth of GaAsP layers has been proposed by Nomura et al. [76]. As with any mixed anion material, the critical issue for GaAsP growth is the control of the group-V composition, especially for high temperatures where As desorption readily occurs.

\subsubsection{Experimental Design}

The investigation of the microscopic processes occurring at the interfaces of MBE grown As/P heterostructures proceeded by implementing a D-optimal designed experiment to systematically characterize the growth of 10-period $\mathrm{GaAs}_{1-\mathrm{y}} \mathrm{P}_{\mathrm{y}} / \mathrm{GaAs}$ strained layer superlattice structures. The incorporation of phosphorus in the SLS structures was achieved by the $\mathrm{P}_{2}$ exposure of an As-stabilized GaAs surface and an immediate overgrowth of GaAs. The statistical experimental design technique implemented for this investigation provided a basis for modeling the phosphorus composition at the interfaces of the SLS structures as a function of substrate temperature $\left(T_{\mathrm{s}}\right)$, phosphorus exposure time $\left(t_{\mathrm{exp}}\right)$, and As-stabilizing flux. The input parameter settings and growth conditions for the designed experiment are given in Table 5.1 and Table 5.2. Epitaxial growth was performed on undoped GaAs (001) epi-ready substrates in a Riber 2300 solid-source molecular beam epitaxy system equipped with a valved As 
cracker and a three-zoned valved $\mathrm{P}$ cracker. The surface reconstructions at the interfaces of the SLS structures were observed during $\mathrm{P}_{2}$ exposure of GaAs using in-situ RHEED, and the structural properties of each SLS were characterized using ex-situ HRXRD.

Table 5.1 Input parameter settings for the anion exchange As/P experimental design.

\begin{tabular}{|l|l|l|l|}
\hline Anion Exchange As/P Experimental Design \\
\hline Parameter Description & Abbreviation & Symbol & Settings \\
\hline Substrate Temperature & $\mathrm{T} \_S u b$ & $T_{\mathrm{s}}$ & $420^{\circ} \mathrm{C}$ to $520^{\circ} \mathrm{C}$ \\
\hline $\mathrm{P}_{2}$ Exposure Time & $\mathrm{P}_{2}$ Sk & $t_{\exp }$ & $10 \mathrm{~s}$ to $50 \mathrm{~s}$ \\
\hline $\mathrm{As}_{4}$ Flux (BEP) & $\mathrm{As}_{4}$ Flux & $P_{\mathrm{As}_{4}}$ & $2 \times 10^{-6}$ Torr, $4 \times 10^{-6} \mathrm{Torr}$ \\
\hline
\end{tabular}

Table 5.2 MBE growth conditions for the $\mathrm{P}_{2}$ exposure of As-stabilized GaAs.

\begin{tabular}{|c|c|c|}
\hline \multicolumn{3}{|c|}{$\mathbf{P}_{2} /$ GaAs Growth Conditions } \\
\hline Growth Condition & Symbol & Settings \\
\hline Ga Flux (BEP) & $P_{\mathrm{Ga}}$ & $1.5 \times 10^{-7}$ Torr \\
\hline $\mathrm{P}_{2}$ Flux (BEP) & $P_{\mathrm{P}_{2}}$ & $4.5 \times 10^{-6}$ Torr \\
\hline GaAs Growth Rate & $v_{\mathrm{GaAs}}$ & $0.5 \mathrm{ML} / \mathrm{s}$ \\
\hline
\end{tabular}

\subsubsection{HRXRD and RSM Analysis}

The phosphorus composition present at the interfaces of the $\mathrm{GaAs}_{1-\mathrm{y}} \mathrm{P}_{\mathrm{y}} / \mathrm{GaAs} \mathrm{SLS}$ structures was determined by performing standard HRXRD $\theta / 2 \theta$ linescans around the substrate (004) reflections. Reciprocal space maps around the (115) reflections were also obtained to investigate the biaxial strain present in these heterostructures. A series of HRXRD $\theta / 2 \theta$ linescans for samples grown over the temperature range of the designed experiment are shown in Figure 5.1. These experiments were performed with an Asstabilizing flux $P_{\mathrm{As}_{4}}=4 \times 10^{-6}$ Torr and phosphorus exposure time $t_{\mathrm{P}}=30 \mathrm{~s}$. Twodimensional equivalent alloy compositions were determined from the average lattice 
mismatch using the zero-order SL peak positions for each x-ray spectra and have been confirmed through dynamical simulation.

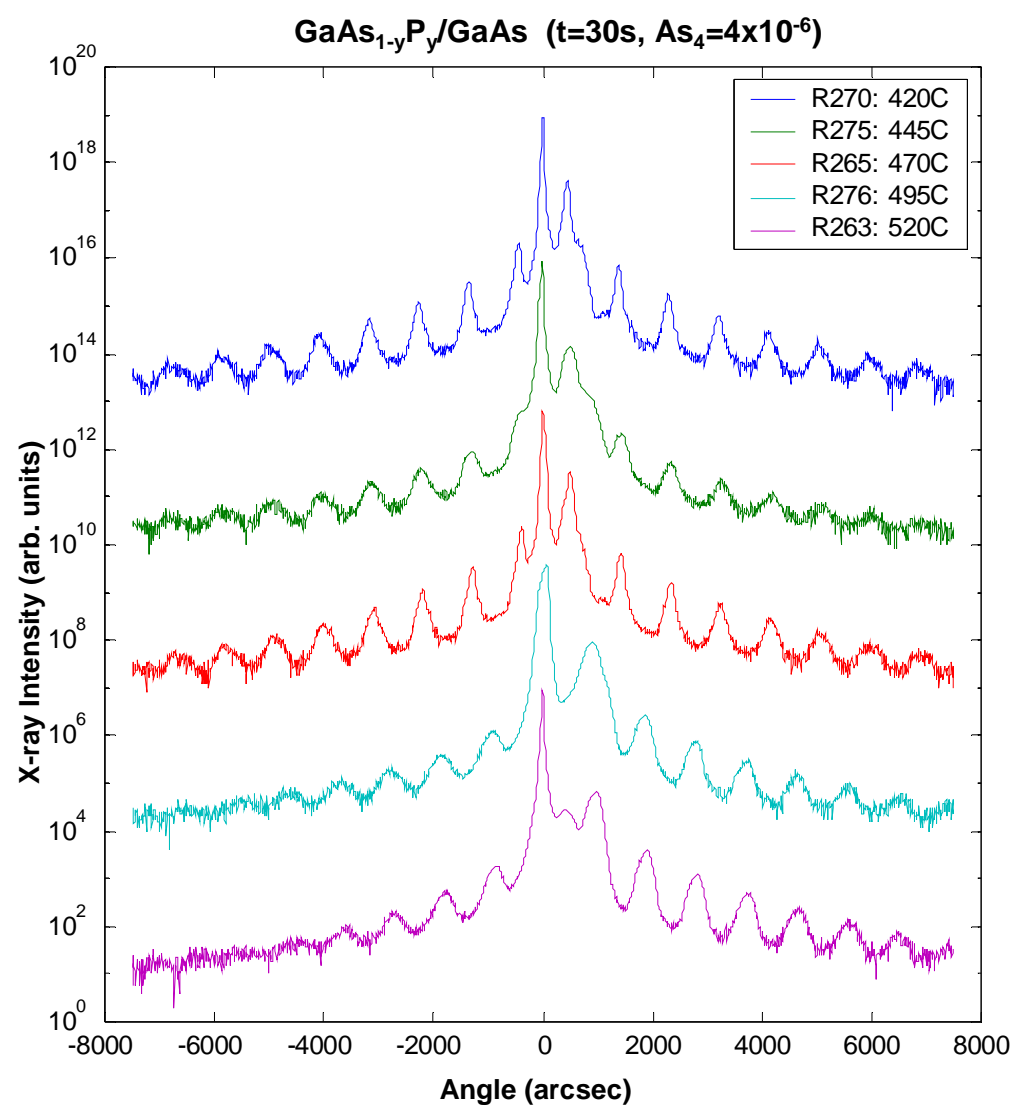

Figure 5.1 HRXRD $\theta / 2 \theta$ linescans for SLS structures formed by the $\mathrm{P}_{2}$-exposure of Asstabilized GaAs surfaces and immediate overgrowth of GaAs.

The HRXRD measurements indicate that the phosphorus composition at the interfaces of the $\mathrm{GaAsP} / \mathrm{GaAs}$ heterostructures increases with substrate temperature, as illustrated in Figure 5.2. These results provide evidence that the phosphorus incorporation at the interface due to P-for-As exchange has a strong non-linear dependence on the substrate temperature. This is in agreement with results obtained by Foxon [77] and other groups investigating the incorporation of phosphorus in GaAsP 
epilayers [51]. Although the GaAsP ternary alloy has the lowest temperature of miscibility among the III-V ternary alloys, the present studies in this system all indicate a strong deviation from linear incorporation [78,79]. Several mechanisms have been proposed to account for the characteristic non-linear incorporation of phosphorus in GaAsP. The dominant theories include anion dimerization [80] and disproportionate sticking probabilities for the group-V elements [81].

\section{$\mathrm{GaAs}_{1-\mathrm{y}} \mathrm{P}_{\mathrm{y}} / \mathrm{GaAs}$ - Average SL Composition Characteristic effect of Ts on SLS $y_{p}$}

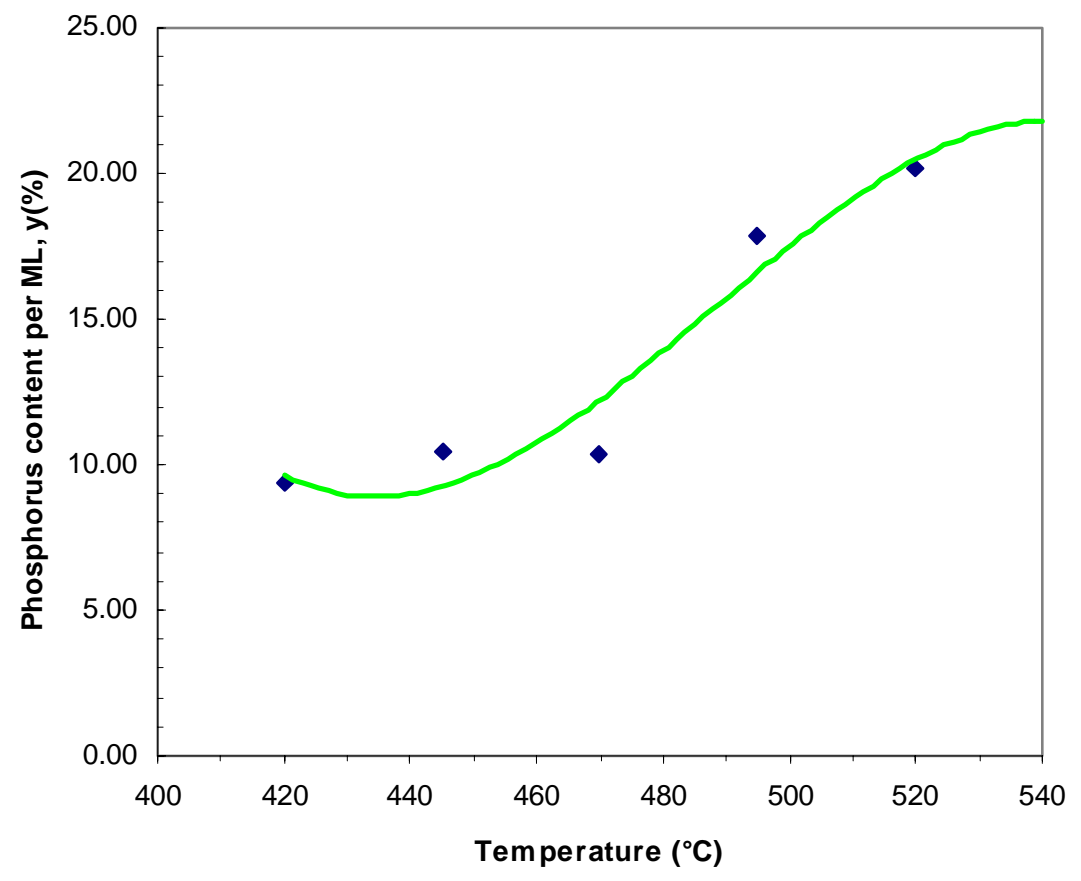

Figure 5.2 The effect of substrate temperature on the phosphorus composition at the interfaces of $\mathrm{GaAsP} / \mathrm{GaAs}$ heterostructures.

The HRXRD $\theta / 2 \theta$ linescans for samples with an As-stabilizing flux $P_{\mathrm{As}_{4}}=2 \times 10^{-6}$ Torr and phosphorus exposure time $t_{\exp }=30 \mathrm{~s}$ grown over the temperature range of the experiment are shown in Figure 5.3. It should be noted that peak broadening was observed for the SL fundamental and satellite peaks of the x-ray spectra for all samples in 
these experiments. Superlattice peak broadening is caused by interfacial roughening, compositional grading, and layer relaxation. Interfacial roughening causes broadening that varies with the square of the satellite order and hence does not broaden the zeroorder peak. Since broadening is observed for the SL fundamental, it is expected that compositional grading is present. When grading and roughness occur together, they can not be distinguished using HRXRD linescans. Similar broadening is observed for relaxed layers, and arises primarily from the dislocation structure. In order to determine if the peak broadening for the GaAsP heterostructures was due to partially strained layers in the SLS, RSMs around the (115) reflections were obtained for each sample.

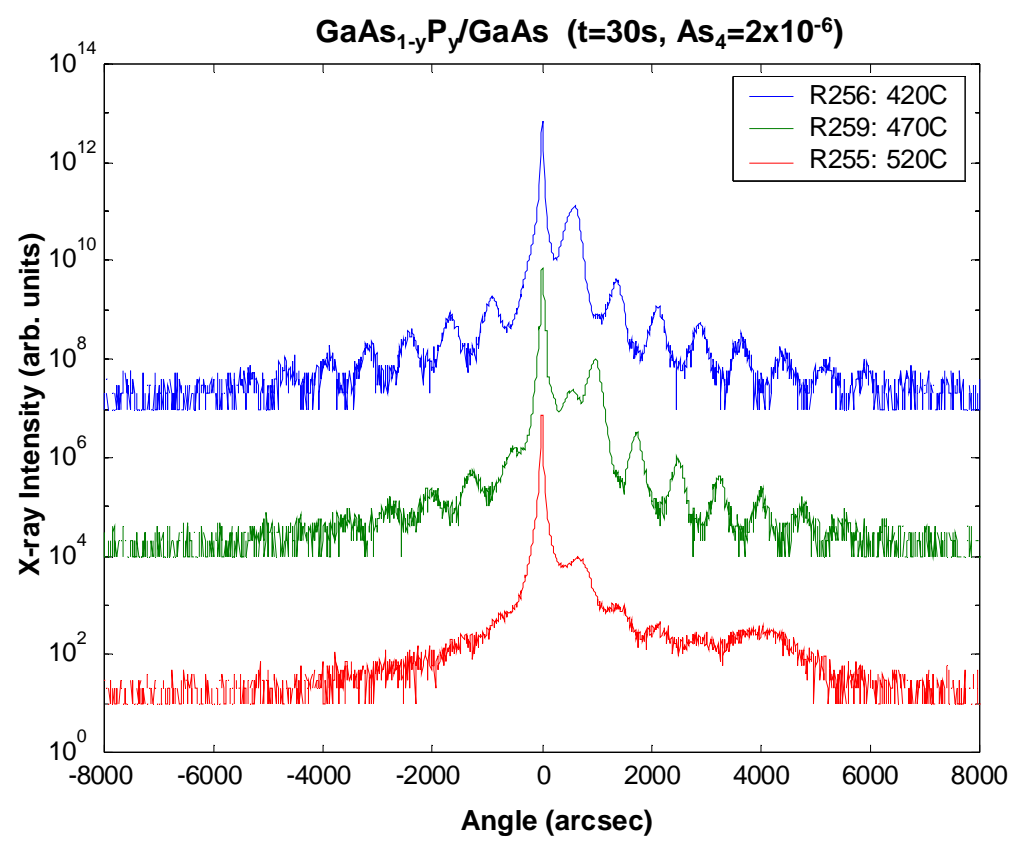

Figure 5.3 HRXRD $\theta / 2 \theta$ linescans for SLS structures formed by the $\mathrm{P}_{2}$-exposure of Asstabilized GaAs surfaces and immediate overgrowth of GaAs.

The RSM data representative of the results obtained for the GaAsP/GaAs heterostructures is shown in Figure 5.4. The interpretation of RSMs is based on the principle that the diffraction peak separation on the horizontal axis $Q_{\mathrm{x}}$ corresponds to 
strain, while the peak separation on the vertical axis $Q_{\mathrm{y}}$ corresponds to lattice mismatch. Diffraction peaks that are vertically aligned are consequently characteristic of pseudomorphic layers. The results obtained for the GaAsP/GaAs SLS indicate that these structures are fully coherent with the GaAs substrate and no strain relaxation is present. The peak broadening observed in the $\mathrm{x}$-ray spectra and RSM data suggest that a thick compositionally graded layer or coherent 3-D islands are formed at the GaAsP/GaAs interface during the phosphorus exposure. The investigations discussed thus far in this research indicate that anion intermixing for these As/P heterostructures can involve several microscopic processes, including anion exchange and diffusion, which could result in compositional variation over many layers at the GaAsP/GaAs interface.

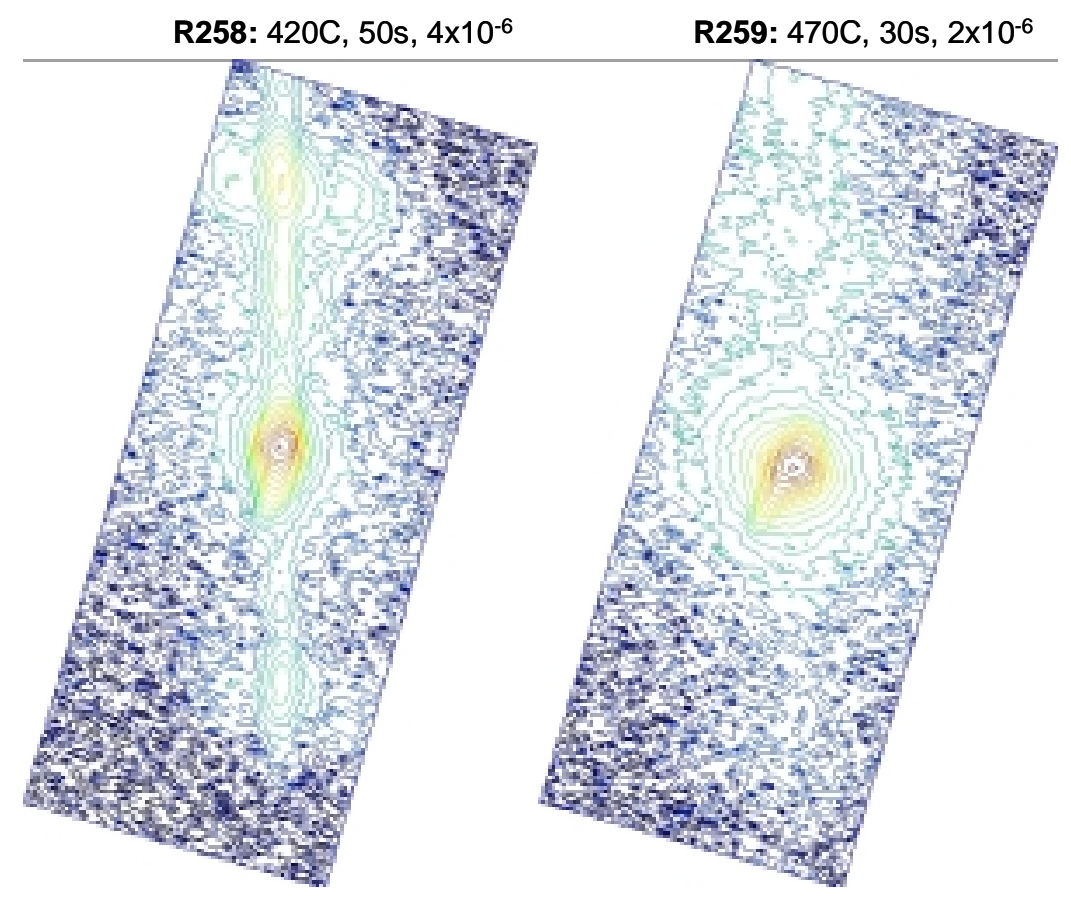

Figure 5.4 Reciprocal space maps around the (115) reflections obtained for GaAsP/GaAs heterostructures. 
The relationship between strain and phosphorus incorporation for the GaAsP/GaAs heterostructures is reasonably straightforward. Based on the RSM data, the difference in lattice parameter between the GaAsP layers and the GaAs buffer layers is accommodated entirely by elastic strain and is defined as

$$
\varepsilon_{0}=2 \frac{a_{f}-a_{s}}{a_{f}+a_{s}} \approx \frac{a_{f}-a_{s}}{a_{s}}=\left(\frac{\Delta a}{a}\right)_{\perp}
$$

This expression represents the unrelaxed strain in the GaAsP layers and is equivalent to the perpendicular lattice mismatch for these SLS structures. The lattice mismatch for experiments with $P_{\mathrm{As}_{4}}=4 \times 10^{-6}$ Torr and $t_{\mathrm{P}}=30 \mathrm{~s}$ are provided in Figure 5.5 and tensile strain is present at the interfaces of the $\mathrm{GaAsP} / \mathrm{GaAs}$ heterostructures.

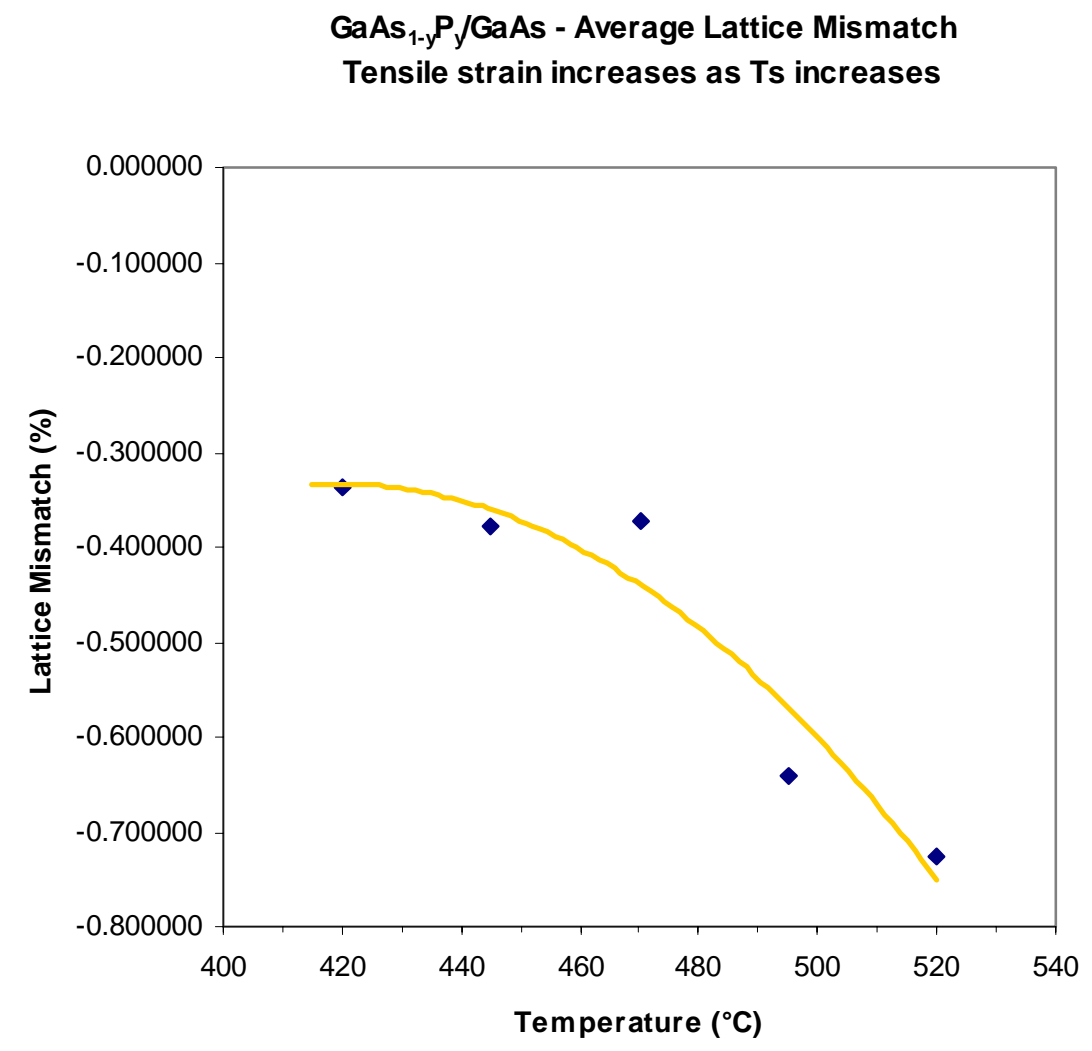

Figure 5.5 The elastic strain in the GaAsP/GaAs SLS structures estimated using the average lattice mismatch. 


\subsubsection{Neural Network Modeling}

The analysis of phosphorus incorporation at the interfaces of $\mathrm{GaAs}_{1-\mathrm{y}} \mathrm{P}_{\mathrm{y}} / \mathrm{GaAs}$ heterostructures required the characterization of the phosphorus composition over the MBE growth regime. This research implemented a neural network modeling technique in order to obtain results for anion intermixing at the interfaces of mixed anion III-V heterostructures. The phosphorus composition data obtained for the D-optimal designed experiment via HRXRD analysis was used to implement a back-propagation (BP) neural network. A three layer BP neural network with a standard 3-5-1 (input-hidden-output) network structure was used to model the phosphorus composition at the interfaces of the GaAsP/GaAs SLS structures. For this model, the input layer corresponded to the experimental parameters, and the output layer corresponded to the phosphorus (dissimilar anion) composition. In order to determine the ability of this model to accurately predict the phosphorus composition, the neural network was trained using $78 \%$ of the experimental data, 18 of the 23 experimental trials selected at random. The remaining five trials were used to test the prediction capability of the network. The neural network model was trained to a root mean square (RMS) error of $4 \%$, and achieved less than $10 \%$ RMS error for test data, implying that the neural network had learned the complex mapping between MBE growth conditions and phosphorus incorporation at the interface.

The neural network model provides fundamental insight into the phosphorus incorporation mechanisms. The dependence of phosphorus composition at the GaAsP/GaAs interface on substrate temperature and phosphorus exposure time is illustrated in Figure 5.6 for an As-stabilizing pressure $P_{\mathrm{As}_{4}}=4 \times 10^{-6}$ Torr and Figure 5.7 for an As-stabilizing pressure $P_{\mathrm{As}_{4}}=2 \times 10^{-6}$ Torr. It is readily seen that the P-for-As 
exchange reaction occurs to a greater extent for samples with an As-stabilizing pressure $P_{\mathrm{As}_{4}}=2 \times 10^{-6}$ Torr compared to samples with an As-stabilizing pressure $P_{\mathrm{As}_{4}}=4 \times 10^{-6}$ Torr. These results indicate that the As-stabilizing flux is a dominant factor for anion intermixing at the interfaces of $\mathrm{GaAsP} / \mathrm{GaAs}$ heterostructures. It should be noted that the non-linear dependence of phosphorus incorporation on substrate temperature is exhibited for the entire range of phosphorus exposure times, and there is a relative increase in Pfor-As exchange for longer anion exposure times.

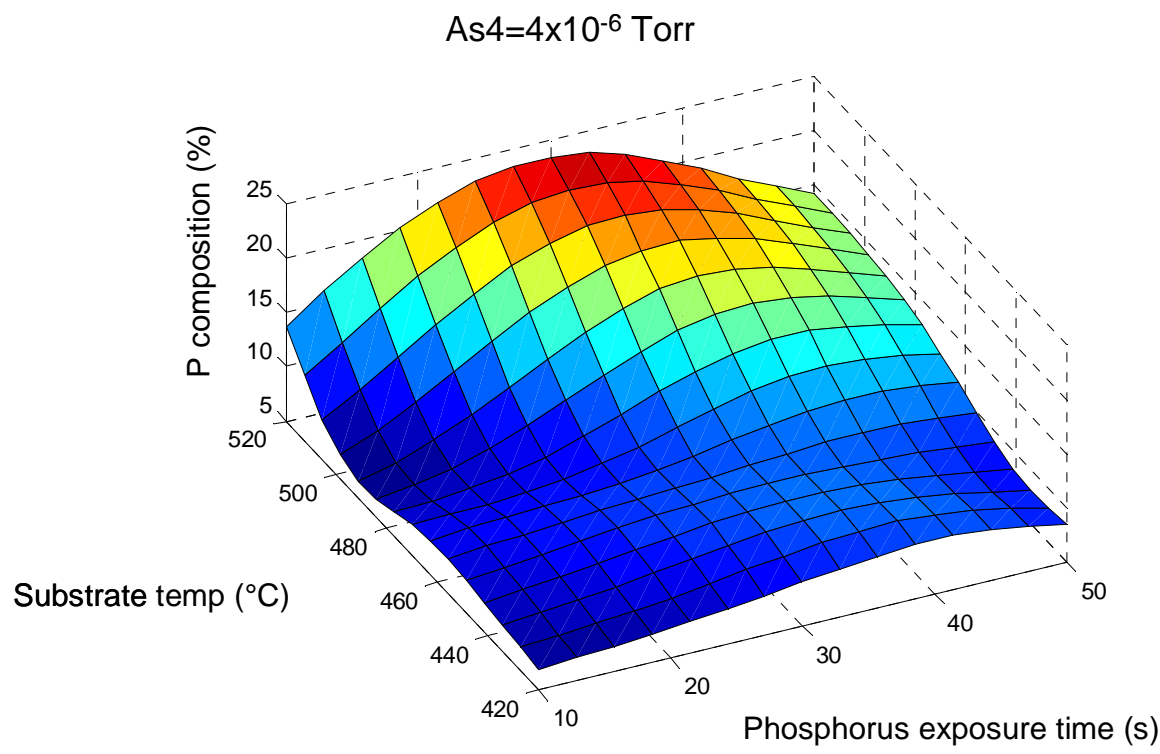

Figure 5.6 Three-dimensional contour plot indicating the dependence of phosphorus composition at the GaAsP/GaAs interface on substrate temperature and phosphorus exposure time.

The phosphorus composition results obtained from the neural network model provide a unique opportunity to determine the primary factors contributing to the phosphorus incorporation mechanism. The sensitivity of the P-for-As exchange process to substrate temperature and anion exposure time was determined using the gradient of the composition data. The results obtained for substrate temperature are provided in 
Figure 5.8. For As-stabilizing pressures $P_{\mathrm{As}_{4}}=4 \times 10^{-6}$ Torr and $P_{\mathrm{As}_{4}}=2 \times 10^{-6}$ Torr, there exist well-defined regions for which the exchange process is invariable to changes in substrate temperature (i.e., $\partial y / \partial T_{\mathrm{s}}=0$ ). Specifically, for $P_{\mathrm{As}_{4}}=2 \times 10^{-6}$ Torr the substrate temperature effects the exchange process only for the growth regime defined by $T_{\mathrm{s}}>$ $490^{\circ} \mathrm{C}$ and $t_{\exp }>25 \mathrm{~s}$. Similar results are obtained for the effects of anion exposure time on the P-for-As exchange process. Therefore, it is apparent that the effects of substrate temperature and phosphorus exposure time on the phosphorus composition at the interface are not as prevalent as that of the As-stabilizing flux. For the GaAsP/GaAs heterostructures, P-for-As anion exchange is more extensive for the low As-stabilizing flux, indicating a dependence of phosphorus incorporation on the static surface structure present before anion exposure or interface formation.

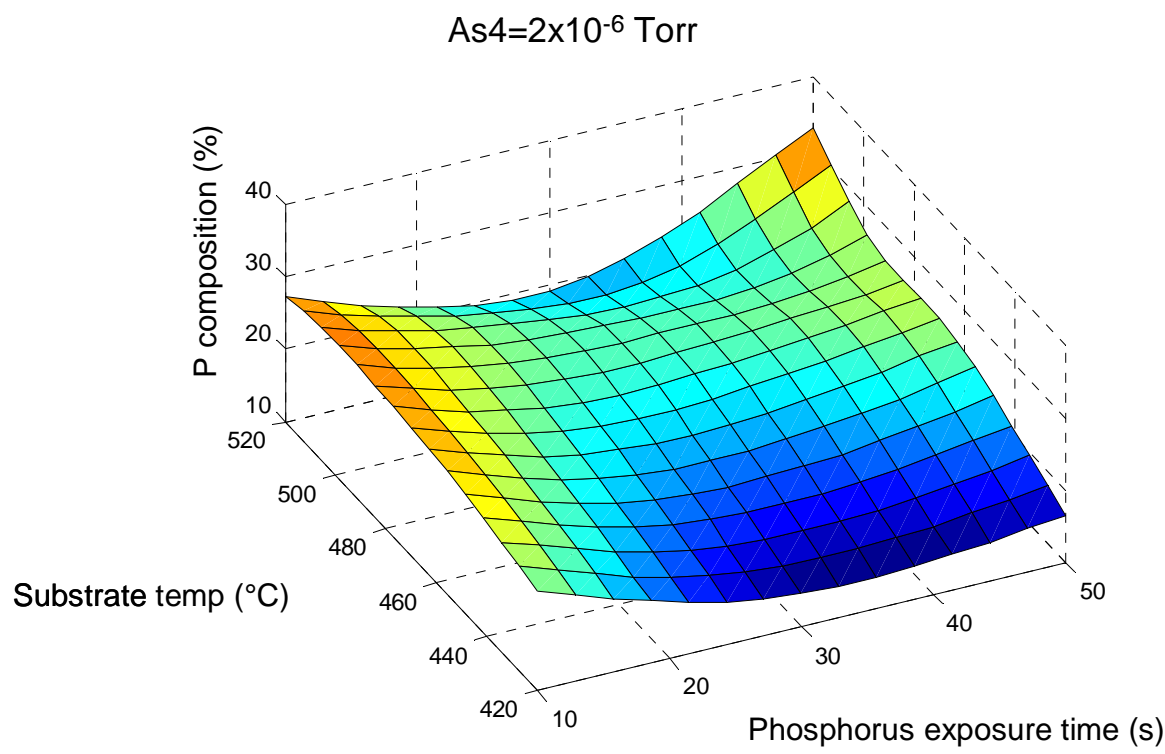

Figure 5.7 Three-dimensional contour plot indicating the dependence of phosphorus composition at the GaAsP/GaAs interface on substrate temperature and phosphorus exposure time. 

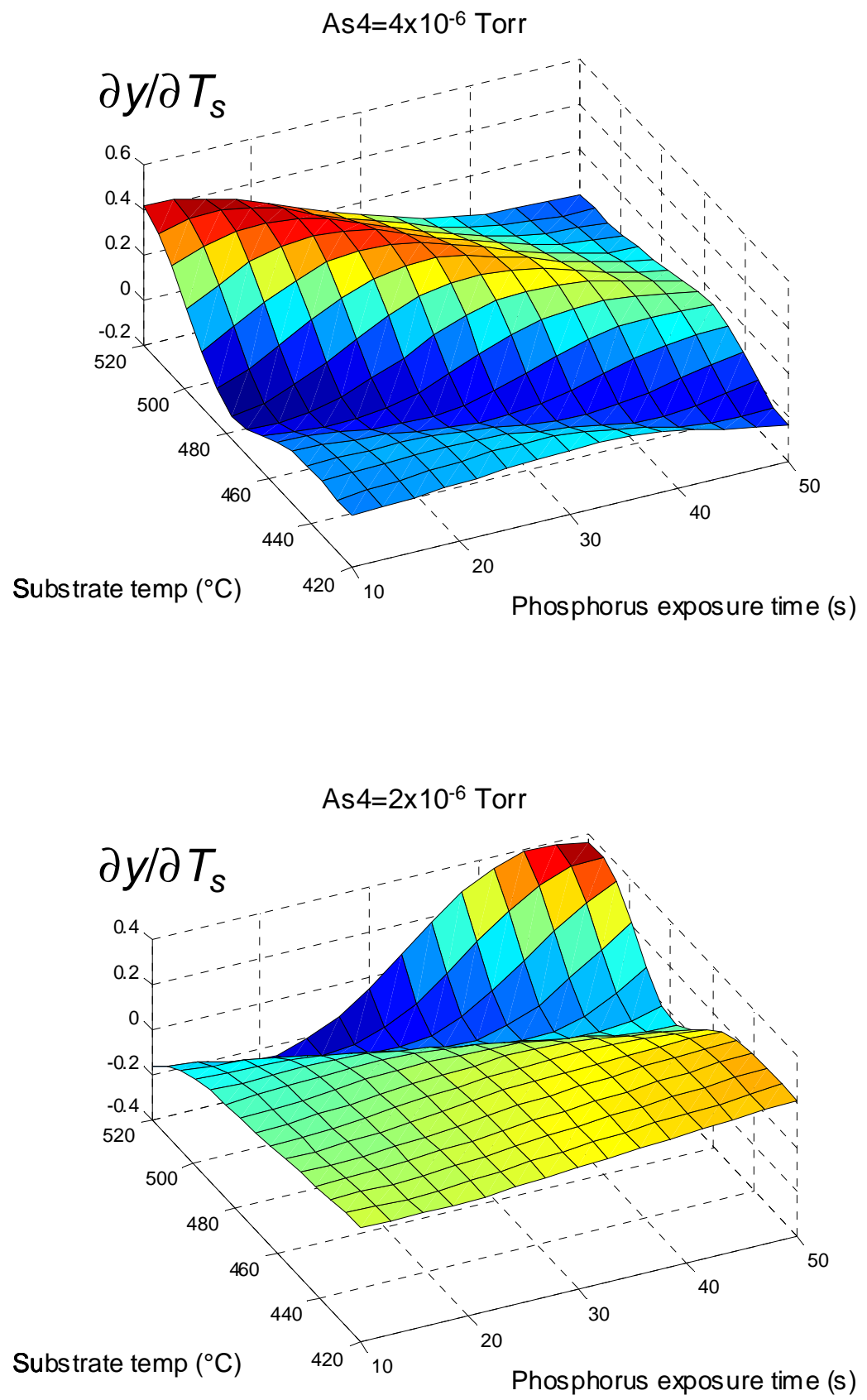

Figure 5.8 Sensitivity of the phosphorus incorporation mechanism with respect to substrate temperature for the high and low As-stabilizing pressures. 


\subsubsection{RHEED and Atomic Surface Structure}

The determination of RHEED characteristic absences has been employed for this research in order to investigate the dependence of anion intermixing at the interfaces of mixed As/P heterostructures on the atomic surface structure of the static As-stabilized GaAs (001) surface. This in-situ surface stoichiometry analysis technique was established by Farrell et al. [39], using kinematic diffraction theory in conjunction with RHEED experiments. These experiments determined that by monitoring the relative intensities of the fractional order RHEED features over the temperature range for MBE growth, the intensity variations observed could be quantified and correlated with specific surface reconstructions. As discussed in Section 3.2, the GaAs $(001)-(2 \times 4)$ pattern exhibits three different contiguous surface phases $(\alpha, \beta$, and $\gamma)$, whose atomic surface structures have been modeled. The results obtained by Farrell et al., illustrated in Figure 5.9, provide a procedure for identifying the three phases of the GaAs $(001)-(2 \times 4)$ surface using the following RHEED characteristic absences: 1) the "least" As-rich $\alpha$ phase is characterized by a relatively weak $2 / 4$ ths diffraction feature, 2) the central $\beta$ phase is characterized by equal intensity among the fourfold fractional order features, and 3) the "most" As-rich $\gamma$ phase is characterized by the absence of the $2 / 4$ ths diffraction feature. This technique provides quantitative $i n$-situ atomic surface structure determination for the MBE growth of GaAs (001) layers.

In order to determine the dependence of anion intermixing at the interfaces of $\mathrm{As} / \mathrm{P}$ heterostructures on atomic surface structure, the RHEED fractional order characterization technique was performed on all SLS structures containing As-stabilized GaAs layers for which RHEED pattern data was acquired. The details of this process, 
along with RHEED data, are provided in Appendix C. The results obtained from this procedure were used to determine the As pressure and substrate temperature transitions for the atomic surface structures, and a map of the As-stabilized GaAs (001) surface reconstructions was constructed. This surface reconstruction map, illustrated in Figure 5.10, provides the fundamental relationships between substrate temperature, Asstabilizing pressure, and the atomic surface structure of the GaAs surface for the MBE growth regime explored in this investigation. The construction of a comprehensive reconstruction map, using the technique described in this section, has been performed by LaBella et al. [82] and shows good agreement with the results obtained here.
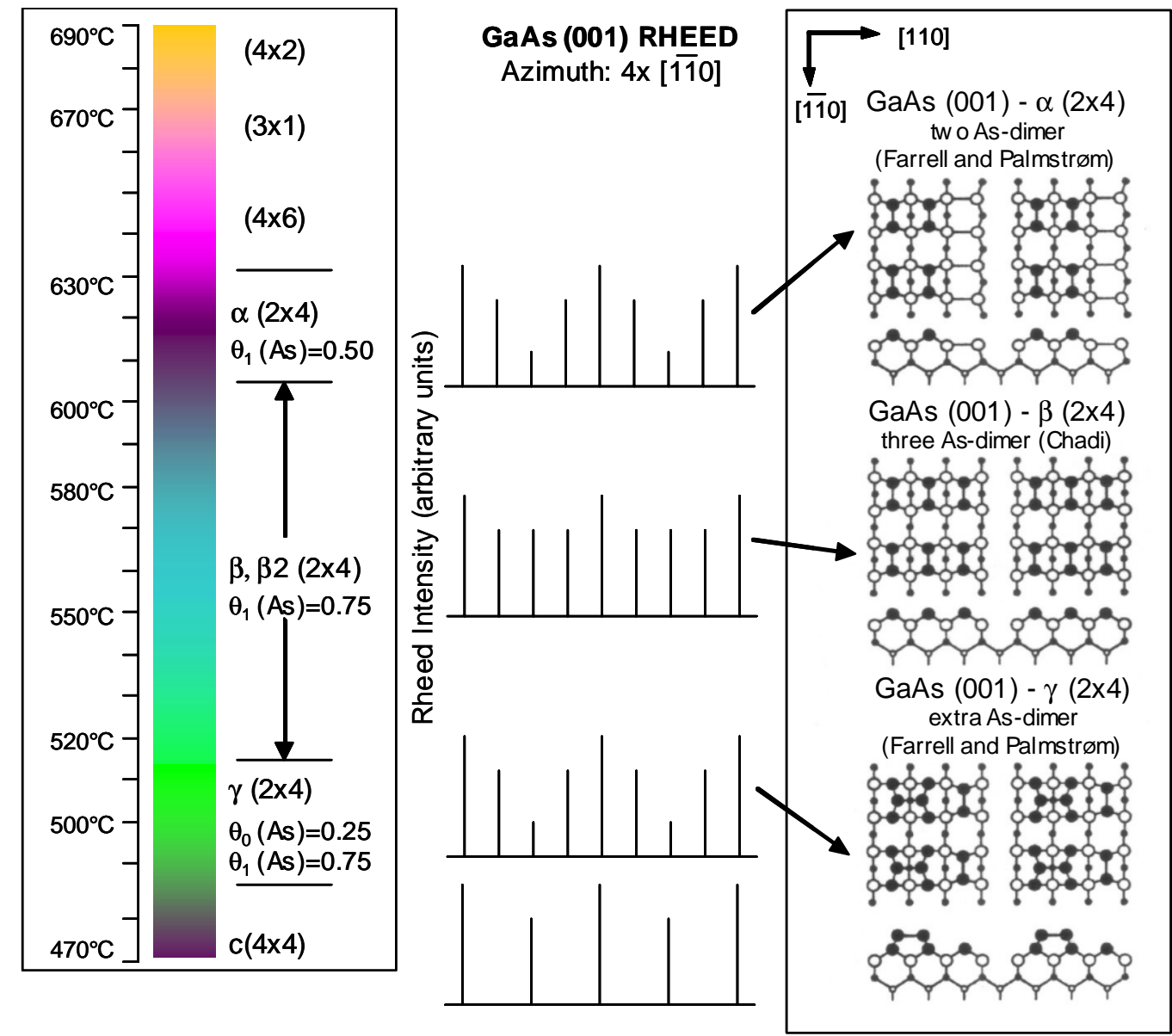

Figure 5.9 RHEED fractional order characterization technique allows in-situ determination of surface stoichiometry and atomic surface structure [39]. 


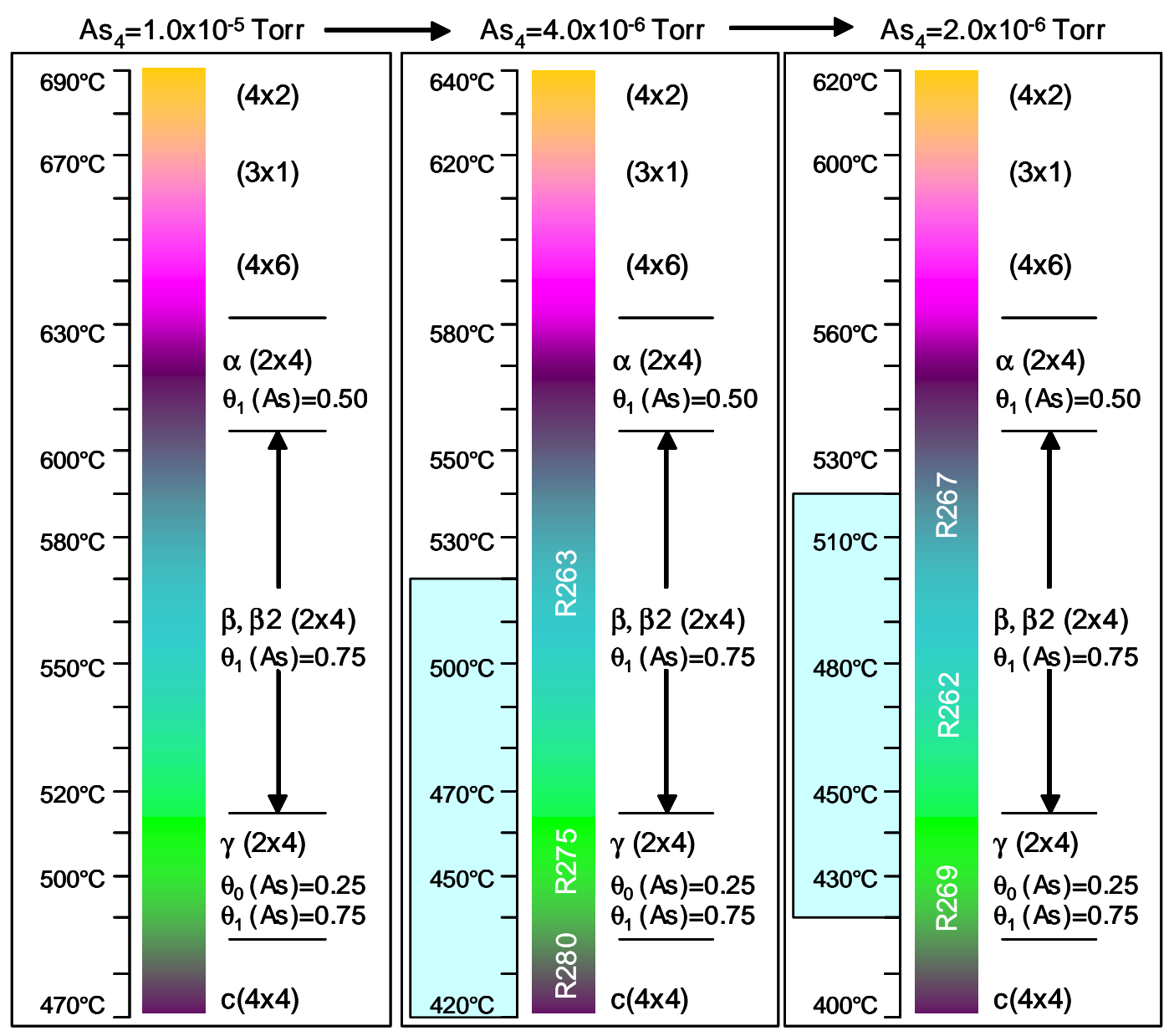

Figure 5.10 Surface reconstruction map for the As-stabilized GaAs (001) surface. 


\subsubsection{Results and Discussion}

The surface reconstruction map for this research has been combined with the neural network modeling results of $\mathrm{P}$-for-As exchange at the interfaces of $\mathrm{GaAsP} / \mathrm{GaAs}$ heterostructures obtained in the previous section. The final results are illustrated in Figure 5.11 for an As-stabilizing flux $P_{\mathrm{As}_{4}}=4 \times 10^{-6}$ Torr and Figure 5.12 for an Asstabilizing flux $P_{\mathrm{As}_{4}}=2 \times 10^{-6}$ Torr. The analysis of these results indicates that several important relationships are present. Specifically, there is a distinct range of phosphorus compositions for each GaAs (001) surface reconstruction, indicating that there exist a strong correlation between phosphorus incorporation and the As-stabilized atomic surface. In general, phosphorus incorporation appears to be inhibited for the GaAs (001)$\mathrm{c}(4 \times 4)$ surface and increases as the surface transitions through the $\gamma, \beta$, and $\alpha$ phases, with the $\alpha$ phase having the largest phosphorus composition, as shown in Figure 5.12. It should be noted that the phosphorus compositions for the $\beta$ and $\gamma$ surface phases are approximately the same for both As-stabilized flux conditions. This provides substantial evidence that the surface reconstruction is the dominant factor for anion intermixing at GaAsP/GaAs interfaces, and the As-stabilizing flux determines only the substrate temperature range for which the atomic surface structures are present. 


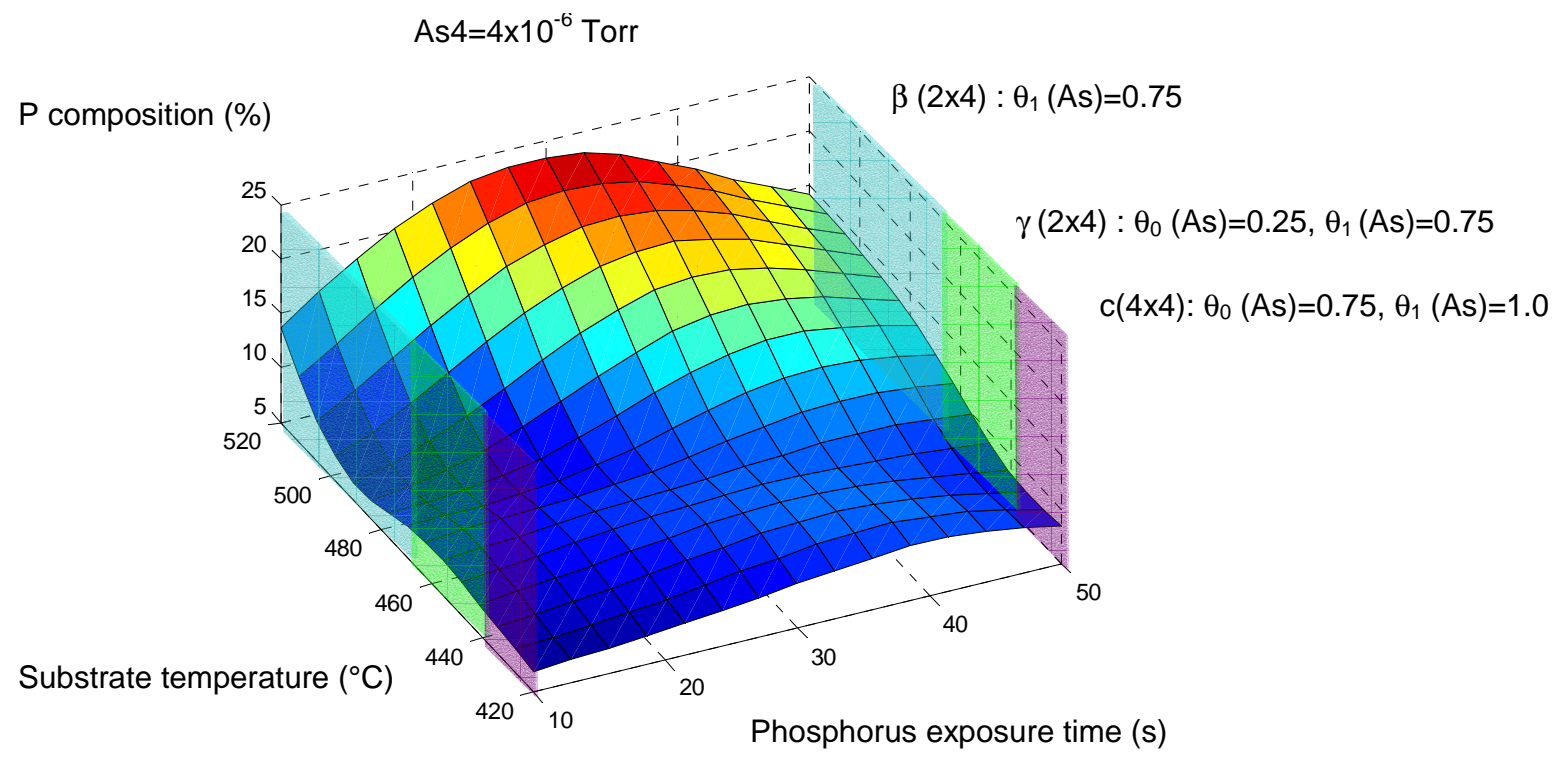

Figure 5.11 Three-dimensional contour plot indicating the dependence of phosphorus composition at the $\mathrm{GaAsP} / \mathrm{GaAs}$ interface on the atomic surface structure of the group- $\mathrm{V}$ stabilized surface.

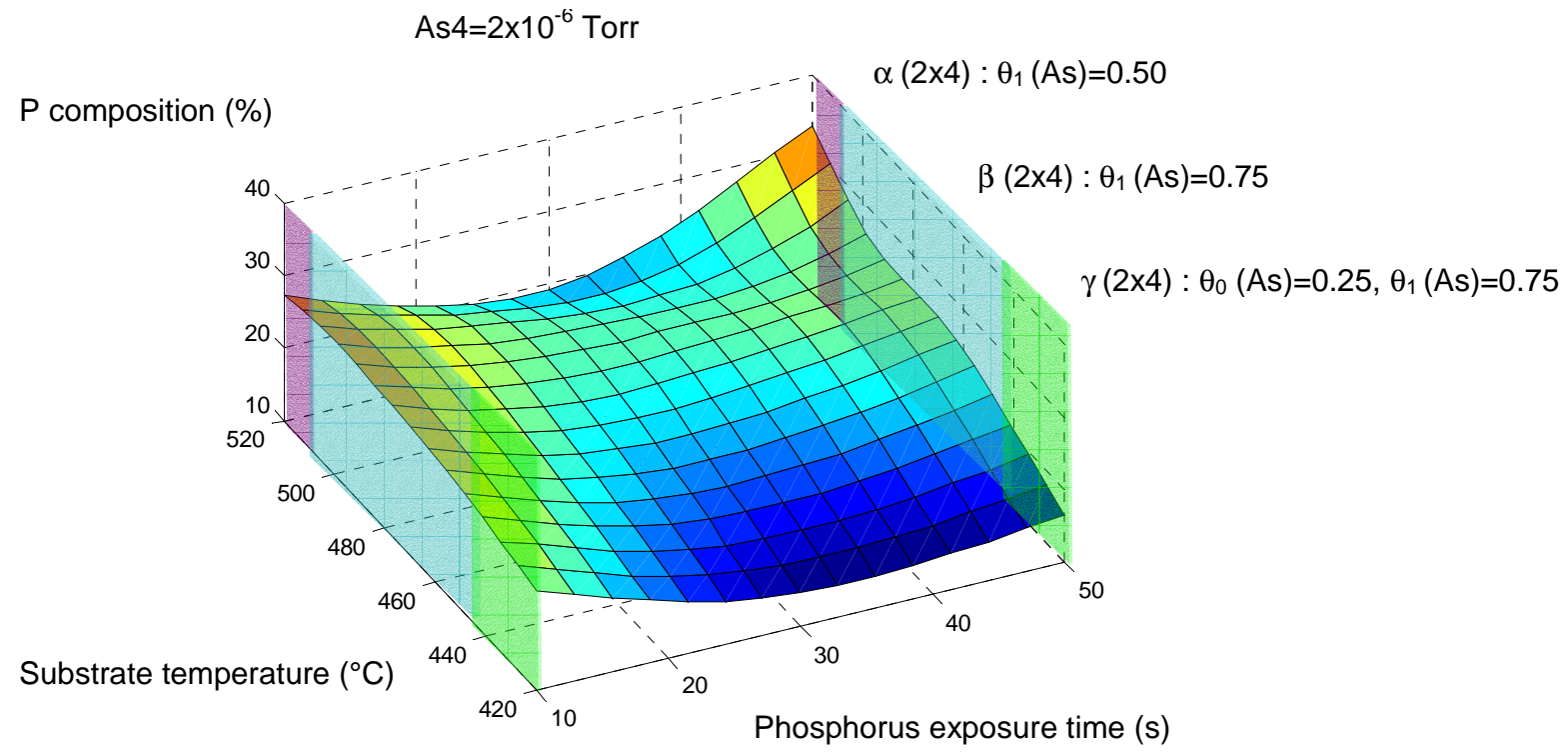

Figure 5.12 Three-dimensional contour plot indicating the dependence of phosphorus composition at the $\mathrm{GaAsP} / \mathrm{GaAs}$ interface on the atomic surface structure of the group- $\mathrm{V}$ stabilized surface. 


\subsection{Summary}

The significance of atomic surface structure for understanding the anion exchange process has been established through the analysis of GaAsP/GaAs interfaces. The phosphorus composition at these interfaces was determined using HRXRD and found to have a strong non-linear dependence on the substrate temperature. A neural network model was constructed to characterize the phosphorus composition with respect to substrate temperature and anion exposure time. The results obtained from the neural network model, in conjunction with RHEED analysis, provided a unique opportunity to determine the dependence of anion exchange on the MBE growth conditions. The investigation revealed significant evidence that the anion exchange process depends primarily on the atomic surface structure present at the interface. The implementation of the hybrid neural network designed to quantify the microscopic processes at the interfaces of mixed anion III-V heterostructures is discussed in the next chapter. 


\section{CHAPTER 6}

\section{ANION EXCHANGE - HYBRID NEURAL NETWORK}

The investigations of anion exchange performed over the course of this research have provided the fundamental knowledge necessary to model the interactions of surface processes and their dependence on MBE growth conditions. The principal objective of this research was achieved by using the understanding of anion exchange, obtained via thin-film characterization and analysis, to construct an advanced process model. The semi-empirical modeling performed for this research identified the contribution of each of the microscopic processes occurring during MBE growth of mixed anion heterostructures. A first-principles kinetic model provided a means for quantifying the effects of anion exchange via kinetic parameters that describe the frequency of surface processes and their probability of occurrence. The focus of this chapter is to discuss the implementation of the hybrid neural network to estimate these kinetic parameters. The algorithms used to construct standard neural networks are introduced, along with the modifications necessary to perform semi-empirical modeling. The implementation of the hybrid neural network is discussed, and its viability as an advanced parameter estimator for complex multivariate modeling is established. The results obtained from the hybrid neural network model are provided and used to analyze the microscopic processes occurring at the interfaces of mixed anion III-V heterostructures. The implementation of the hybrid neural network was performed for the GaAsP/GaAs heterostructure system. 


\subsection{Hybrid Neural Network Modeling}

The semi-empirical hybrid neural network model developed for this research incorporates a first principles kinetic model with a back-propagation neural network. In this way, the hybrid model combines the best aspects of physical and empirical modeling methods. The kinetic model, expressed in Equation (6.1), describes the interactions of the processes occurring at mixed anion interfaces from physical considerations, while the neural network complements this model by estimating the unknown process parameters.

$$
\begin{aligned}
& y\left(T_{s}, t_{\exp }, \Phi\right)=s \Phi \tau\left[1-\exp \left(-t_{\text {exp }} / \tau\right)\right] \times\left[1+2 \sqrt{D t_{\text {exp }} / \pi}\right] \\
& \tau \equiv\left(\tau_{d}^{-1}+s \Phi\right)^{-1}, \tau_{d}^{-1}=\tau_{0 d}^{-1} \exp \left(-E_{d} / k_{B} T_{s}\right), D=D_{0} \exp \left(-E_{a} / k_{B} T_{s}\right)
\end{aligned}
$$

Kinetic Parameters: $s, \tau_{0 d}, E_{d}, D_{0}, E_{a}$

The efforts performed to derive the first-principles kinetic model in Equation (6.1) were discussed in Section 3.4. The physical definitions of each of the variables incorporated into the model are provided in Table 6.1. The primary surface processes of interest for the $\mathrm{GaAsP} / \mathrm{GaAs}$ heterostructure system are anion exchange, surface desorption, and diffusion. The kinetic model describes the complete growth sequence for the SLS structures, and has been expressed here such that it accounts for only those surface processes that are known to occur for the As/P system.

Table 6.1 The physical definitions for each of the variables used in the kinetic model.

\begin{tabular}{|l|l|l|l|}
\hline P-for-As Anion Exchange Kinetic Model Definitions \\
\hline Description & Abbreviation & Symbol & Units \\
\hline Phosphorus Composition & P $_{2}$ Comp & $y$ & percent \\
\hline Desorption Rate Constant & Des_Const & $\tau_{\mathrm{d}}$ & seconds \\
\hline Diffusion Rate Constant & Diff_Coeff & $D$ & $\mathrm{~cm}^{2} /$ second \\
\hline Sticking Coefficient & Stick_Coeff & $s$ & $\mathrm{~cm}^{2}$ \\
\hline
\end{tabular}




\subsubsection{Experimental Design}

The hybrid neural network model was constructed by characterizing the MBE growth of $\mathrm{As} / \mathrm{P}$ heterostructures using a statistically designed experiment. The $\mathrm{GaAsP} / \mathrm{GaAs}$ SLS structures are formed by allowing a $\mathrm{P}_{2}$ flux to impinge on a static Asstabilized GaAs (001) surface. The phosphorus composition $\left(y_{\mathrm{a}}\right)$ at the interfaces of these structures is modeled as a function of substrate temperature $\left(T_{\mathrm{s}}\right)$, phosphorus exposure time $\left(t_{\text {exp }}\right)$, and arsenic stabilizing flux $\left(\Phi_{\mathrm{N}}\right)$. The details of the P-for-As anion exchange experiment were discussed in Section 5.1. The growth conditions and factor settings for the D-optimal designed experiment are provided in Table 6.2.

Table 6.2 Input parameter settings for the anion exchange As/P experimental design.

\begin{tabular}{|l|l|l|l|}
\hline P-for-As Anion Exchange Experimental Design \\
\hline Factor Description & Abbreviation & Symbol & Settings \\
\hline Substrate Temperature & $\mathrm{T} \_$Sub & $T_{\mathrm{s}}$ & 420 to $520^{\circ} \mathrm{C}$ \\
\hline $\mathrm{P}_{2}$ Exposure Time & $\mathrm{P}_{2}$ Sk & $\mathrm{t}_{\mathrm{exp}}$ & 10 to $50 \mathrm{~s}$ \\
\hline $\mathrm{P}_{2}$ Exposure Flux (BEP) & $\mathrm{P}_{2}$ Flux & $P_{\mathrm{P}_{2}}$ & $4.5 \times 10^{-6} \mathrm{Torr}$ \\
\hline $\mathrm{As}_{4}$ Stabilizing Flux (BEP) & $\mathrm{As}_{4}$ _Flux & $P_{\mathrm{As}_{4}}$ & $2 \times 10^{-6} \mathrm{Torr}, 4 \times 10^{-6} \mathrm{Torr}$ \\
\hline Experimental $\mathrm{P}_{2}$ Composition & Exp_Comp & $y_{\mathrm{a}}$ & 0 to 100 percent \\
\hline
\end{tabular}

It should be noted that the kinetic model depends directly on the quantity of each of the constituent elements present on the surface during both As stabilization and phosphorus exposure of the static surface. Therefore, the group-V pressures must be represented as molecular fluxes. The relationship for a molecular species $(i)$ between beam equivalent pressure $\left(P_{i}\right)$ under ultra-high vacuum conditions and the flux $\left(\omega_{i}\right)$ of molecules reaching the surface of the substrate is expressed as

$$
\omega_{i}=P_{i} \sqrt{\frac{N_{A}}{2 \pi \cdot k_{B} M_{i} \cdot T}} \text { molecules } / \mathrm{m}^{2} \cdot \mathrm{s}
$$


where $T$ is the temperature, $M_{i}$ is the molecular weight, and $N_{\mathrm{A}}$ and $k_{\mathrm{B}}$ are the Avogadro and Boltzmann constants, respectively. For the phosphorus exposure pressure $P_{\mathrm{P}_{2}}=$ $4.5 \times 10^{-6}$ Torr and phosphorus evaporation temperature $T=92^{\circ} \mathrm{C}$, Equation (6.2) can be evaluated using SI units, resulting in a phosphorus flux $\Phi=1.051 \times 10^{15} \mathrm{molecules} / \mathrm{cm}^{2} \cdot \mathrm{s}$.

\subsubsection{Hybrid Neural Network Architecture}

The neural networks used in this research are multilayer feed-forward perceptron networks. The structure of such a feed-forward neural network is illustrated in Figure 6.1. These networks consist of multiple layers of neurons - an input layer, several hidden layers, and an output layer. The input layer neurons correspond to the input parameters or experimental conditions, while the output neurons provide the overall response of the network. Individual neurons are interconnected in such a way that the weights of the connections between them are the free parameters of the network. This massively parallel interconnection allows neural networks to resolve arbitrary nonlinear mappings.

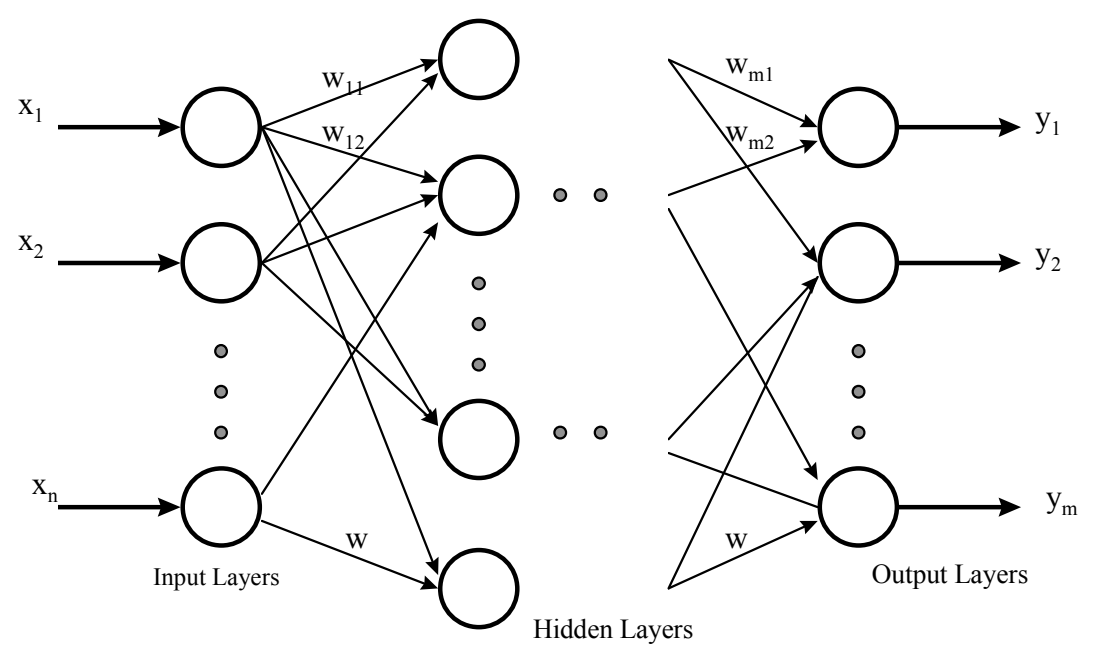

Figure 6.1 The structure for the multilayer feed-forward neural network showing input, hidden, and output layers. 


\subsubsection{Standard Neural Network Algorithm}

The most common method of learning for feed-forward neural networks is the error back-propagation (BP) algorithm, which is classified as a supervised learning technique. The initial step for learning is to set the weights (free parameters) of the network to small random numbers that are uniformly distributed. The BP algorithm consists of two phases, the forward propagation phase and the back-propagation phase, and requires that the network be trained using a set of input/output data having $n$ training vectors $(d(n): n=1,2,3, \ldots, N)$, whose functional relationship the network attempts to learn. The forward propagation phase requires that the input vectors be presented to the network and propagated through the layers of the network. The functionality of each neuron is to compute the weighted sum of its inputs and determine its activation level using a non-linear function. This process can be analytically expressed as

$$
\begin{aligned}
& x_{i}^{(k)}(n)=\sum_{j} w_{i, j}^{(k)}(n) \cdot y_{j}^{(k-1)}(n) \\
& y_{i}^{(k)}(n)=\frac{1}{1+\exp \left(-x_{i}^{(k)}(n)\right)}
\end{aligned}
$$

where $w_{i, j}^{(k)}$ is the synaptic weight, $x_{i}^{(k)}$ is the input, and $y_{i}^{(k)}$ is the output corresponding to the $i^{\text {th }}$ neuron in the $k^{\text {th }}$ layer. The nonlinear activation function used to determine the output of each neuron enables neural networks to generalize with a degree of freedom not available in statistical techniques [56]. The activation level of the neurons in the output layer determines the predicted response of the network. After the outputs $\left(o_{i}(n)\right)$ of this final layer are computed, they are compared to the desired or measured output data $\left(d_{i}(n)\right)$, generating an error signal that is expressed as $e_{i}(n)=d_{i}(n)-o_{i}(n)$. The mean 
squared training error $(E)$ of the network is computed by taking the sum of the squared errors over all of the neurons in the output layer:

$$
E=\frac{1}{2} \sum_{i} e_{i}^{2}(n)=\frac{1}{2} \sum_{i}\left(d_{i}(n)-o_{i}(n)\right)^{2}
$$

After the forward propagation phase is completed, the back-propagation phase proceeds by minimizing the network error via modification of the weights, one layer at a time. The mathematical approach for error minimization is a first-order gradient descent method, which adjust the network weights by an amount proportional to the derivative of the error with respect to the previous weights. The first step in this process involves computing the local gradients $\left(\delta_{i}^{(k)}\right)$ of the network. These gradients are the Euclidean distance in weight space which the network attempts to minimize. They are defined as

$$
\delta_{i}^{(k)}=-\frac{\partial E}{\partial x_{i}^{(k)}}=\left(\frac{\partial E}{\partial y_{i}^{(k)}}\right)\left(\frac{\partial y_{i}^{(k)}}{\partial x_{i}^{(k)}}\right)
$$

and are determined by propagating the error backward through the network, resulting in

$$
\begin{aligned}
& \delta_{i}^{(k)}=e_{i}(n)\left[1-o_{i}^{2}(n)\right] \\
& \delta_{i}^{(k)}=\left\lfloor 1-\left(y_{i}^{(k)}(n)\right)^{2}\right\rfloor \cdot \sum_{l} \delta_{l}^{(k+1)}(n) \cdot w_{l, j}^{(k+1)}(n)
\end{aligned}
$$

for neurons in the output layer, Equation (6.7), and neurons in hidden layers, Equation (6.8). The gradient of the error with respect to the previous weights $\left(\partial E / \partial w_{i, j}^{(k)}\right)$ is a sensitivity factor and determines the direction of search in weight space for the synaptic weight $w_{i, j}^{(k)}$. This gradient can be derived from the above equations and is expressed as

$$
\frac{\partial E}{\partial w_{i, j}^{(k)}}=\left(\frac{\partial E}{\partial x_{i}^{(k)}}\right)\left(\frac{\partial x_{i}^{(k)}}{\partial w_{i, j}^{(k)}}\right)=-\delta_{i}^{(k)} \cdot y_{j}^{(k-1)}
$$


The minus sign in this expression accounts for gradient descent in weight space, and this result is used to construct the synaptic weight correction mechanism, known as the delta rule. The delta rule defines the change in synaptic weight $w_{i, j}^{(k)}$ and is given by

$$
\begin{aligned}
& w_{i, j}^{(k)}(n+1)=w_{i, j}^{(k)}(n)+\Delta w_{i, j}^{(k)} \\
& \Delta w_{i, j}^{(k)}=-\eta \frac{\partial E}{\partial w_{i, j}^{(k)}}
\end{aligned}
$$

where $\eta$ is a proportionality constant called the learning-rate. The gradient of the error with respect to the weights is calculated for one input/output training vector at a time. In this way, the synaptic weights are updated such that the predicted responses of the network approach the desired responses. After the network weights are updated, the procedure is repeated for the set of input/output data until convergence is achieved.

\subsubsection{Hybrid Neural Network Training Algorithm}

The structure of the hybrid neural network model designed to predict anion intermixing for the GaAsP/GaAs heterostructures is illustrated in Figure 6.2. The neural network component of the hybrid model has the MBE process conditions as its inputs. The outputs of the neural network component are the unknown parameters required to implement the kinetic model. The neural network component consists of two backpropagation neural networks in parallel. These 3-layer neural networks are implemented using 3-4-3 (input-hidden-output neuron) and 3-4-2 network structures, where each network uses all of the input factors to estimate a subset of the model parameters. An important issue in neural network modeling is the effect of the size of the available training data set on the accuracy of the learned model. The general rule for ensuring a neural network's ability to resolve complex nonlinear mappings is that the number of free 
parameters in the network should not exceed the number of training vectors. When this condition is not satisfied the network may be under-constrained, resulting in a strong dependence on interpolation. The parallel neural network architecture developed for this research provides a 50 to $58 \%$ reduction in the number of free parameters compared to a 3-6-5 network structure and drastically improves convergence. It should be noted that while a standard neural network relies only on training data to perform mapping, a hybrid neural network already contains a partial model. This reduces the error signal to a subspace that can be sampled sufficiently using fewer training vectors and coupled with the advantages of parallel networks, results in improved generalization and extrapolation.

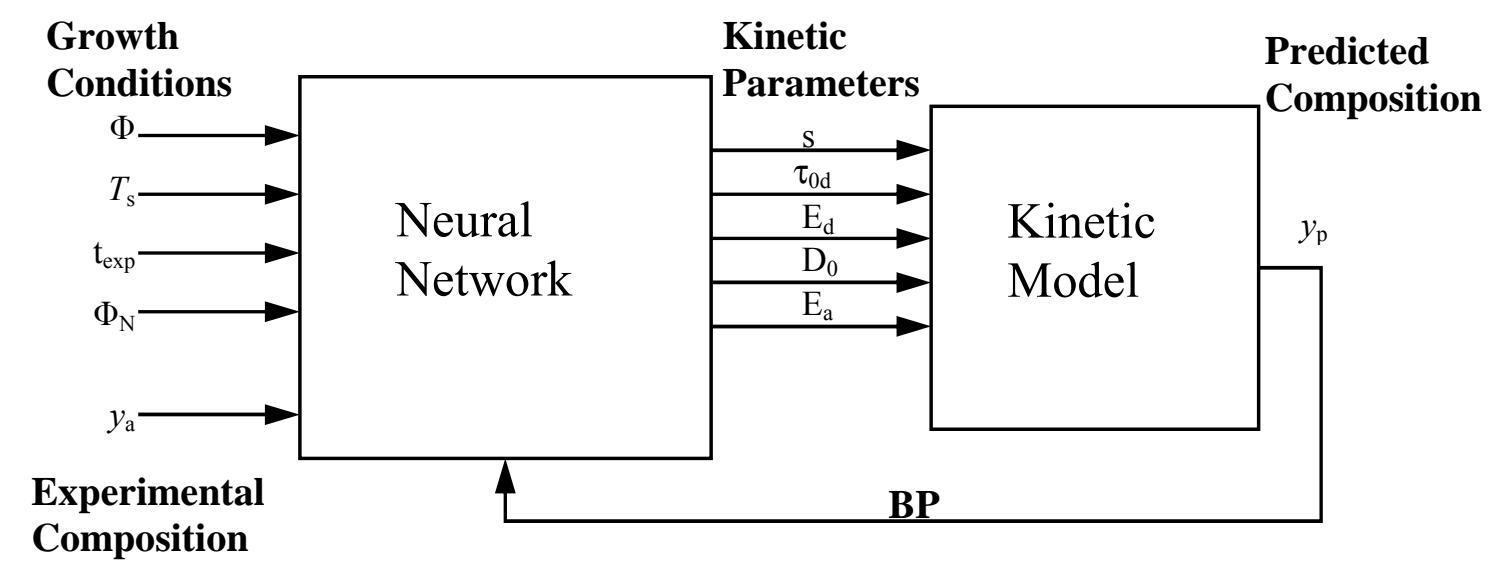

Figure 6.2 The structural implementation of the hybrid neural network capable of estimating the kinetic parameters for the As/P heterostructures.

The BP algorithm detailed in the previous section is the method of learning implemented for the hybrid neural network model. It should be noted that network learning for this modeling technique is designed to determine an appropriate set of network weights for the neural network component to facilitate process parameter estimation. The forward and back-propagation phases of the BP algorithm proceed in a 
manner similar to that of standard neural networks. Network training occurs by means of a modified error gradient that takes into account the error contribution from each kinetic parameter determined by the partial derivatives of the kinetic model [83].

The initial step for learning is to set the weights of the neural network component to small random numbers. After initializing the neural network component, the forward propagation phase begins by presenting the input vectors to the hybrid neural network. The estimated parameters corresponding to the outputs of the neural network component are the inputs to the kinetic model. These unknown physical constants are used to compute the predicted phosphorus composition at the interfaces of the $\mathrm{GaAsP} / \mathrm{GaAs}$ heterostructures. This predicted composition is the final output of the hybrid neural network and is used to determine the network error. The predicted composition $\left(y_{p}(n)\right)$ is compared to the experimental phosphorus composition $\left(y_{a}(n)\right)$, producing an error signal that is expressed as $e(n)=y_{a}(n)-y_{p}(n)$. The mean squared prediction error $(E)$ of the hybrid neural network is computed using this error signal and is expressed as

$$
E=\frac{1}{2} e^{2}(n)=\frac{1}{2}\left(y_{a}(n)-y_{p}(n)\right)^{2}
$$

The back-propagation phase proceeds by minimizing the network error via modification of the synaptic weights. The gradient descent method is the approach used for error minimization and is analytically the same as that for standard neural networks. The first step is to compute the local gradients $\left(\delta_{i}^{(k)}\right)$ by propagating the error backward through the hybrid neural network. The network error can be propagated backward through the kinetic model component, essentially by computing the partial derivatives of the kinetic model with respect to each of the process parameters. Using this procedure, 
the contribution of each kinetic parameter to the hybrid neural network error is obtained and used to calculate modified local gradients for the neural network component. These modified local gradients define the distance in weight space that the hybrid neural network model attempts to minimize and are expressed as

$$
\delta_{i}^{(k)}=-\frac{\partial E}{\partial x_{i}^{(k)}}=\left(\frac{\partial E}{\partial y_{p}}\right)\left(\frac{\partial y_{p}}{\partial y_{i}^{(k)}}\right)\left(\frac{\partial y_{i}^{(k)}}{\partial x_{i}^{(k)}}\right)
$$

where $\left(\partial E / \partial y_{p}\right)$ is the error signal $e(n)=y_{a}(n)-y_{p}(n)$, and $\left(\partial y_{i}^{(k)} / \partial x_{i}^{(k)}\right)$ is the current derivative of the network output used in the standard BP algorithm. The partial derivative defined by $\left(\partial y_{p} / \partial y_{i}^{(k)}\right)$ is computed separately for each kinetic parameter and represents the modification necessary to perform semi-empirical modeling. The local gradients for the neural network component are used to derive the gradient of the error with respect to the previous weights $\left(\partial E / \partial w_{i, j}^{(k)}\right)$. This gradient is required to construct the weight correction mechanism and is expressed as

$$
\frac{\partial E}{\partial w_{i, j}^{(k)}}=\left(\frac{\partial E}{\partial x_{i}^{(k)}}\right)\left(\frac{\partial x_{i}^{(k)}}{\partial w_{i, j}^{(k)}}\right)=\left(\frac{\partial E}{\partial y_{p}}\right)\left(\frac{\partial y_{p}}{\partial y_{i}^{(k)}}\right)\left(\frac{\partial y_{i}^{(k)}}{\partial x_{i}^{(k)}}\right)\left(\frac{\partial x_{i}^{(k)}}{\partial w_{i, j}^{(k)}}\right)=-\delta_{i}^{(k)} \cdot y_{j}^{(k-1)}
$$

It is apparent from this expression that the partial derivatives of the kinetic model account for the contribution of each kinetic parameter to the network error. The delta rule defined by the standard BP algorithm is also used for the hybrid neural network, and the synaptic weights are updated such that the predicted responses approach the desired responses. This modified BP algorithm is repeated for the set of input/out data until convergence is achieved, at which point the kinetic parameters are known explicitly. The partial derivatives for the five unknown parameters of the first principles kinetic model developed for this research are provided in Equations (6.14) through (6.18). 


$$
\begin{aligned}
& \frac{\partial y}{\partial s}=1 / 2 \Phi \tau_{d}\left(\pi+2 \sqrt{\pi \cdot D t_{\exp }}\right)\left(1-h_{1}+s \Phi t_{\exp } h_{1}+s^{2} \Phi^{2} t_{\exp } h_{1} \tau_{d}\right) \cdot \pi^{-1}\left(1+s \Phi \tau_{d}\right)^{-2} \\
& \frac{\partial y}{\partial \tau_{0 d}}=r\left(1-\exp \left(-t_{\exp } / \tau\right)\right) \cdot h_{2} \tau^{2}\left(\tau_{0 d} \cdot \tau_{d}\right)^{-1}-r t_{\exp } \exp \left(-t_{\exp } / \tau\right) \cdot h_{2} \tau\left(\tau_{0 d} \cdot \tau_{d}\right)^{-1} \\
& \frac{\partial y}{\partial E_{d}}=r\left(1-\exp \left(-t_{\exp } / \tau\right)\right) \cdot h_{2} \tau^{2}\left(\tau_{d} \cdot k_{B} T_{s}\right)^{-1}-r t_{\exp } \exp \left(-t_{\exp } / \tau\right) \cdot h_{2} \tau\left(\tau_{d} \cdot k_{B} T_{s}\right)^{-1} \\
& \frac{\partial y}{\partial D_{0}}=1 / 2 r \tau\left(1-\exp \left(-t_{\exp } / \tau\right)\right) \cdot D t_{\exp }\left(D_{0} \pi \sqrt{D t_{\exp } / \pi}\right)^{-1} \\
& \frac{\partial y}{\partial E_{a}}=-1 / 2 r \tau\left(1-\exp \left(-t_{\exp } / \tau\right)\right) \cdot D t_{\exp }\left(k_{B} T_{s} \pi \sqrt{D t_{\exp } / \pi}\right)^{-1} \\
& r=s \Phi, h_{1}=\exp \left(-t_{\exp }\left(1+s \Phi \tau_{d}\right) / \tau_{d}\right), h_{2}=1 / 2+\sqrt{D t_{\exp } / \pi}
\end{aligned}
$$

\subsubsection{Kinetic Model Parameter Estimation}

The hybrid neural network estimates the following unknown process parameters: the sticking coefficient $(s)$, desorption coefficient and its associated activation energy $\left(\tau_{0 \mathrm{~d}}\right.$, $\left.E_{\mathrm{d}}\right)$, and diffusion coefficient and its activation energy $\left(D_{0}, E_{\mathrm{a}}\right)$. The physical ranges for these kinetic parameters are provided in Table 6.3. These parameter ranges define the boundaries or limits of validity for the physical model.

Table 6.3 The physical ranges of the kinetic parameters that are estimated by the hybrid neural network model.

\begin{tabular}{|l|l|l|}
\hline P-for-As Anion Exchange Kinetic Parameters \\
\hline Description & Symbol & Physical Range \\
\hline Desorption Coefficient & $\tau_{0 \mathrm{~d}}$ & 0 to $10 \mathrm{~s}$ \\
\hline Desorption Activation Energy & $E_{d}$ & 0 to $5 \mathrm{eV}$ \\
\hline Diffusion Coefficient & $D_{0}$ & 0 to $80 \mathrm{~cm}^{2} / \mathrm{sec}$ \\
\hline Diffusion Activation Energy & $E_{a}$ & 0 to $5 \mathrm{eV}$ \\
\hline Sticking Coefficient & $s$ & 0 to $1 \mathrm{~cm}^{2}$ \\
\hline
\end{tabular}


In order to obtain estimates for the kinetic model parameters, the measured phosphorus composition $\left(y_{a}\right)$ was used as an input to the hybrid neural network during training. This provides the neural network component with prior knowledge of the desired hybrid neural network response, and facilitates parameter estimation for the kinetic model component. The parameter estimation results obtained during hybrid neural network training are provided in Table 6.4 for an As-stabilizing flux $P_{\mathrm{As}_{4}}=4 \times 10^{-6}$ Torr and Table 6.5 for an As-stabilizing flux $P_{\mathrm{As}_{4}}=2 \times 10^{-6}$ Torr. It should be noted that each entry in these tables represents a training vector or experimental trial. Therefore, the substrate temperature $\left(T_{s}\right)$, phosphorus exposure time $\left(t_{\exp }\right)$, measured and predicted phosphorus composition, and parameter estimates are provided for each vector.

Table 6.4 The hybrid neural network parameter estimations for $\mathrm{As}_{4}=4 \times 10^{-6}$ Torr.

\begin{tabular}{|c|c|c|c|c|c|c|c|c|}
\multicolumn{8}{|c|}{ Parameter Estimation - $\mathbf{A s} \mathbf{4}=\mathbf{4 \times 1 0 ^ { - 6 }}$ Torr } \\
\hline$T_{\mathrm{s}}$ & $\mathrm{t}_{\exp }$ & $y_{\mathrm{a}}$ & $y_{\mathrm{p}}$ & $s$ & $\tau_{0 \mathrm{~d}}$ & $E_{\mathrm{d}}$ & $D_{0}$ & $E_{\mathrm{a}}$ \\
\hline$\left({ }^{\circ} \mathrm{C}\right)$ & $(\mathrm{s})$ & $(\%)$ & $(\%)$ & $\left(\mathrm{cm}^{2}\right)$ & $(\mathrm{s})$ & $(\mathrm{eV})$ & $\left(\mathrm{cm}^{2} / \mathrm{s}\right)$ & $(\mathrm{eV})$ \\
\hline 420 & 50 & 8.39 & 8.390089 & 0.342846 & 6.766507 & 2.925813 & 18.88093 & 0.184673 \\
\hline 420 & 10 & 7.08 & 7.080224 & 0.447021 & 6.585642 & 2.527076 & 17.86789 & 0.108546 \\
\hline 470 & 50 & 11.39 & 11.39027 & 0.309196 & 6.387 & 3.165246 & 17.87287 & 0.150838 \\
\hline 420 & 30 & 9.34 & 9.339524 & 0.377816 & 6.735592 & 2.82534 & 18.21261 & 0.137565 \\
\hline 470 & 10 & 7.69 & 7.68942 & 0.450963 & 6.469017 & 2.558437 & 18.20244 & 0.105334 \\
\hline 520 & 10 & 13.63 & 13.62989 & 0.394033 & 6.150161 & 2.919911 & 17.09242 & 0.021235 \\
\hline 445 & 30 & 10.48 & 10.48005 & 0.368312 & 6.622955 & 2.905669 & 17.78163 & 0.125179 \\
\hline 495 & 30 & 17.85 & 17.85015 & 0.304093 & 6.231352 & 3.324152 & 15.06194 & 0.046752 \\
\hline 520 & 50 & 14.18 & 14.18032 & 0.281095 & 6.034265 & 3.368671 & 17.0747 & 0.125348 \\
\hline 520 & 30 & 20.16 & 20.16015 & 0.285573 & 6.046485 & 3.430027 & 14.73495 & 0.02921 \\
\hline 470 & 30 & 9.63 & 9.630063 & 0.37282 & 6.497513 & 2.889369 & 18.15639 & 0.142906 \\
\hline
\end{tabular}

For an ideal system (i.e., a non-linear function that is well defined and differentiable, along with a dataset containing no noise) parameter estimation should result in the same solution for all training vectors. The hybrid neural network model 
developed for this research was initially tested using a known response defined by the non-linear function $F(x, y)=\alpha \cdot x^{2}+\beta \cdot y^{2}$, where $x$ and $y$ were the experimental conditions (inputs), and $\alpha$ and $\beta$ were the parameters being estimated. This function was evaluated using a random set of inputs along with predetermined parameter ranges, and the ideal case was obtained, providing verification of the hybrid neural network implementation and establishing its viability as an advanced parameter estimator.

Table 6.5 The hybrid neural network parameter estimations for $\mathrm{As}_{4}=2 \times 10^{-6}$ Torr.

\begin{tabular}{|c|c|c|c|c|c|c|c|c|}
\hline \multicolumn{9}{|c|}{ Parameter Estimation $-\mathbf{A s}_{4}=2 \times 10^{-6}$ Torr } \\
\hline$T_{\mathrm{s}}$ & $t_{\text {exp }}$ & $y_{\mathrm{a}}$ & $y_{p}$ & $s$ & $\tau_{0 \mathrm{~d}}$ & $E_{\mathrm{d}}$ & $D_{0}$ & $E_{\mathrm{a}}$ \\
\hline$\left({ }^{\circ} \mathrm{C}\right)$ & $(\mathrm{s})$ & $(\%)$ & $(\%)$ & $\left(\mathrm{cm}^{2}\right)$ & (s) & $(\mathrm{eV})$ & $\left(\mathrm{cm}^{2} / \mathrm{s}\right)$ & $(\mathrm{eV})$ \\
\hline 520 & 30 & 19.25 & 19.24996 & 0.365109 & 4.280078 & 2.541532 & 29.08873 & 0.082355 \\
\hline 420 & 30 & 14.62 & 14.61991 & 0.332716 & 2.715344 & 2.21908 & 16.93086 & 0.074601 \\
\hline 520 & 10 & 27.24 & 27.2401 & 0.430642 & 5.100752 & 2.836114 & 56.16052 & 0.002583 \\
\hline 520 & 50 & 34.81 & 34.80994 & 0.414387 & 4.436666 & 2.470239 & 27.6142 & 0.029421 \\
\hline 470 & 30 & 19.4 & 19.40016 & 0.352965 & 3.50258 & 2.402748 & 21.61391 & 0.05709 \\
\hline 420 & 50 & 16.47 & 16.47033 & 0.33944 & 2.426094 & 2.039185 & 15.77891 & 0.085687 \\
\hline 470 & 30 & 24.18 & 24.18002 & 0.373941 & 3.713711 & 2.462066 & 23.50593 & 0.032884 \\
\hline 420 & 10 & 15.08 & 15.08 & 0.339765 & 3.14971 & 2.445438 & 25.25683 & 0.028897 \\
\hline
\end{tabular}

Experimentally, it is not always possible to obtain the exact solution for all training vectors, and as expected, the kinetic parameter results obtained for this research do not reflect ideal behavior. This is due primarily to the inherent noise in the measured experimental conditions and measured responses (e.g. obtaining slightly different responses for identical measured experimental conditions). It also should be noted that the variability in the kinetic parameter estimates for the hybrid neural network model are minimal, but may indicate that the kinetic model does not completely describe the mechanisms that control the surface processes being modeled. 


\subsubsection{Results and Discussion}

Evaluation of the trained hybrid neural network model is performed in terms of root mean squared error (RMSE), computed as the square root of the network prediction error $(E)$. The hybrid neural network implemented for samples with an As-stabilizing flux $P_{\mathrm{As}_{4}}=4 \times 10^{-6}$ Torr demonstrated a training RMSE of $0.028 \%$ and a prediction RMSE of $0.574 \%$. Figure 6.3 illustrates the measured phosphorus composition versus the predicted composition for these samples, indicating the accuracy of the hybrid neural process model. Similar results were obtained for the samples with an As-stabilizing flux $P_{\mathrm{As}_{4}}=2 \times 10^{-6}$ Torr. The measured phosphorus composition versus the predicted composition for these samples is illustrated in Figure 6.4.

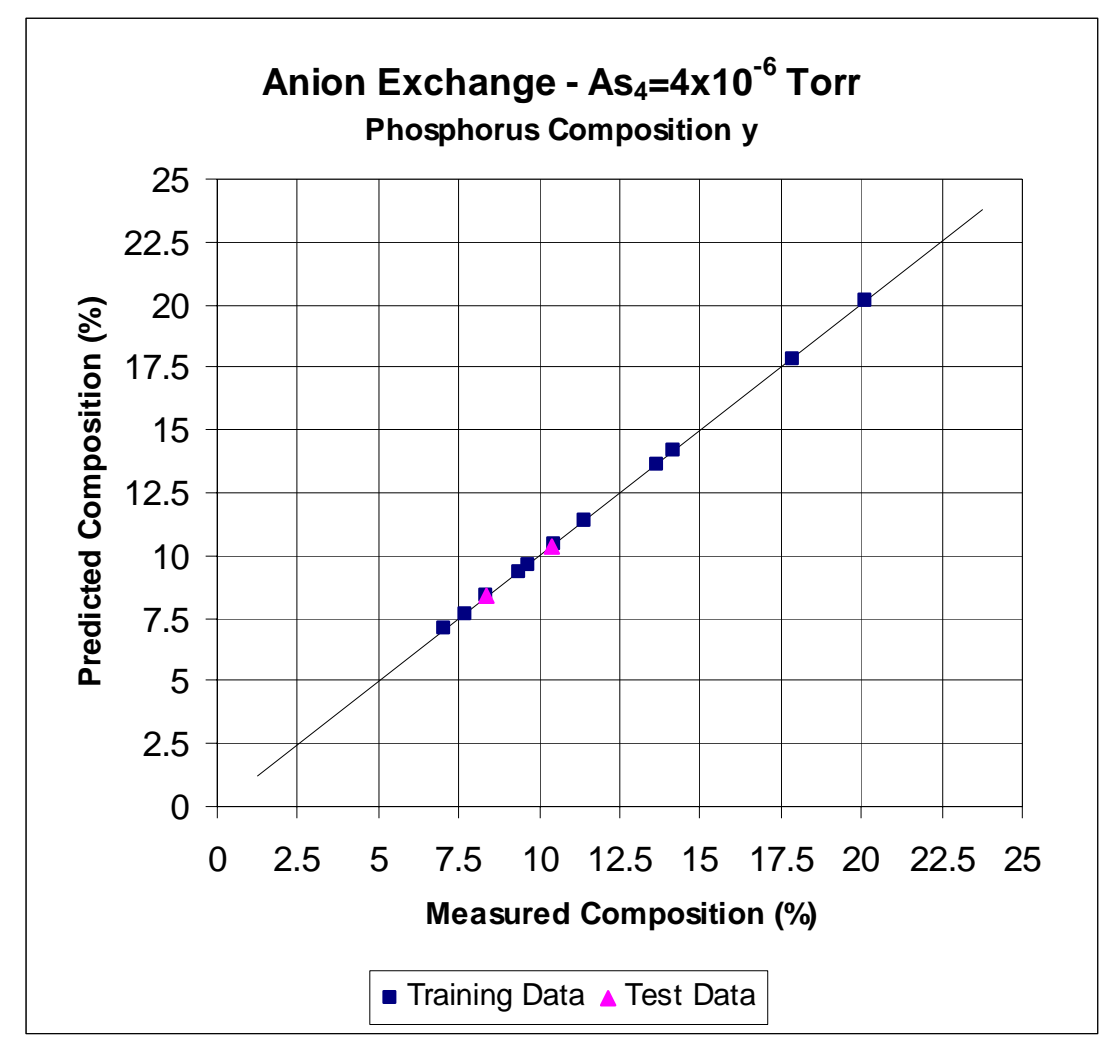

Figure 6.3 Experimental phosphorus composition versus the neural network predictions for $\mathrm{As}_{4}=4 \times 10^{-6}$ Torr. 


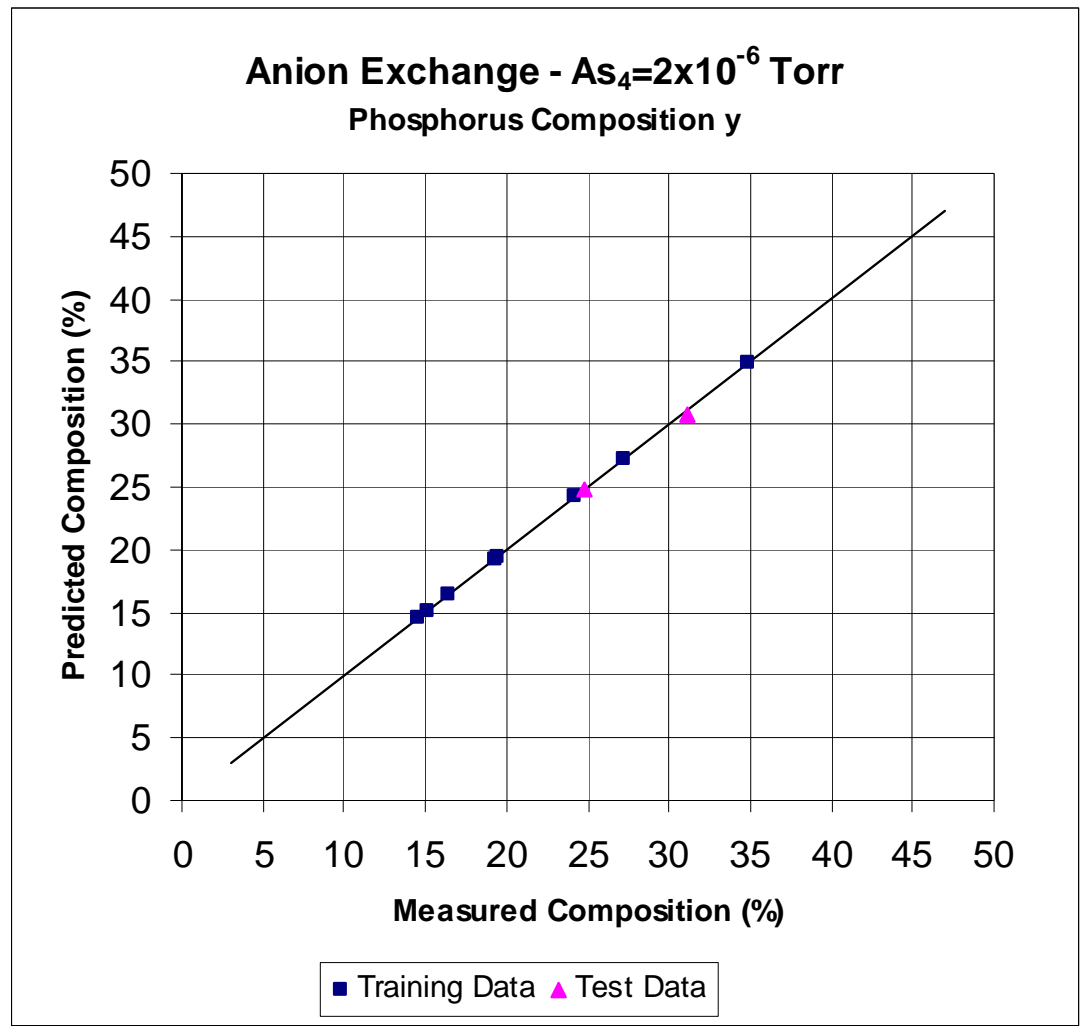

Figure 6.4 Experimental phosphorus composition versus the neural network predictions for $\mathrm{As}_{4}=2 \times 10^{-6}$ Torr.

In order to obtain the final kinetic parameters required to implement the first principles kinetic model, a mean filter was applied to the estimated parameters obtained during hybrid neural network training. The kinetic parameters obtained for this research are provided in Table 6.6 for samples with an As-stabilizing flux $P_{\mathrm{As}_{4}}=4 \times 10^{-6}$ Torr and Table 6.7 for samples with an As-stabilizing flux $P_{\mathrm{As}_{4}}=2 \times 10^{-6}$ Torr. Using these estimated process parameters, the kinetic model can predict the phosphorus composition of the mixed arsenic/phosphorus III-V heterostructures.

The hybrid neural network results are important for analyzing the microscopic processes occurring at the interfaces of the $\mathrm{GaAsP} / \mathrm{GaAs}$ heterostructures. The kinetic 
parameters for the two distinct As-stabilizing fluxes were compared, and several interesting relationships were evident. As discussed in Section 5.1.4, the higher As flux corresponds to atomic surface structures with increased surface arsenic coverage. Taking this into account, it is immediately apparent from the hybrid neural network results that the phosphorus sticking coefficient for samples with $P_{\mathrm{As}_{4}}=4 \times 10^{-6}$ Torr is slightly lower than those with $P_{\mathrm{As}_{4}}=2 \times 10^{-6}$ Torr. This is expected and likely due to the relative increase in As surface coverage corresponding to surface structures such as the $c(4 \times 4)$. It should also be noted that theoretical estimates of group-V sticking coefficients predict values of less than $0.5 \mathrm{~cm}^{2}$, and the results obtained here are in good agreement [77].

Table 6.6 The kinetic parameters obtained by the semi-empirical hybrid neural network model for $\mathrm{As}_{4}=4 \times 10^{-6}$ Torr.

\begin{tabular}{|c|c|c|c|c|}
\hline \multicolumn{6}{|c|}{ Kinetic Parameters $-\mathbf{A} \mathbf{s}_{\mathbf{4}}=\mathbf{4 \times 1 0 ^ { - 6 }}$ Torr } \\
\hline$s$ & $\tau_{0 \mathrm{~d}}$ & $E_{\mathrm{d}}$ & $D_{0}$ & $E_{\mathrm{a}}$ \\
\hline$\left(\mathrm{cm}^{2}\right)$ & $(\mathrm{s})$ & $(\mathrm{eV})$ & $\left(\mathrm{cm}^{2} / \mathrm{s}\right)$ & $(\mathrm{eV})$ \\
\hline 0.357615 & 6.411499 & 2.985428 & 17.35807 & 0.107053 \\
\hline
\end{tabular}

For the phosphorus surface desorption process, a lower activation energy and increased reaction frequency are observed for the $P_{\mathrm{As}_{4}}=2 \times 10^{-6}$ Torr samples. These parameter results indicate an unexpected increase in phosphorus desorption for the $P_{\mathrm{As}_{4}}=$ $2 \times 10^{-6}$ Torr samples. While it is unclear what mechanism drives this process, it should be noted that the surface structures for the $P_{\mathrm{As}_{4}}=2 \times 10^{-6}$ Torr samples occur at relatively higher temperatures than those for the $P_{\mathrm{As}_{4}}=4 \times 10^{-6}$ Torr samples. These results support the idea that surface desorption is primarily dependent on substrate temperature. 
The dominant microscopic process occurring for the GaAsP/GaAs samples is phosphorus diffusion. As expected, the diffusion mechanism occurs more rapidly for the $P_{\mathrm{As}_{4}}=2 \times 10^{-6}$ Torr samples and has a lower activation energy. This indicates that the phosphorus diffusion mechanism for the $P_{\mathrm{As}_{4}}=4 \times 10^{-6}$ Torr samples occurs to a greater extent than for the $P_{\mathrm{As}_{4}}=2 \times 10^{-6}$ Torr samples. The hybrid neural network results obtained demonstrate excellent agreement with the RHEED and HRXRD results obtained in this research. This is a strong indication that the hybrid neural network accurately predicts the contribution of each of the microscopic processes occurring at the interfaces of the mixed anion III-V heterostructures.

Table 6.7 The kinetic parameters obtained by the semi-empirical hybrid neural network model for $\mathrm{As}_{4}=2 \times 10^{-6}$ Torr.

\begin{tabular}{|c|c|c|c|c|}
\multicolumn{6}{|c|}{ Kinetic Parameters - $\mathbf{A s}_{\mathbf{4}}=\mathbf{2 \times 1 0 ^ { - 6 }}$ Torr } \\
\hline$S$ & $\tau_{0 \mathrm{~d}}$ & $E_{\mathrm{d}}$ & $D_{0}$ & $E_{\mathrm{a}}$ \\
\hline$\left(\mathrm{cm}^{2}\right)$ & $(\mathrm{s})$ & $(\mathrm{eV})$ & $\left(\mathrm{cm}^{2} / \mathrm{s}\right)$ & $(\mathrm{eV})$ \\
\hline 0.368621 & 3.665617 & 2.42705 & 26.99374 & 0.04919 \\
\hline
\end{tabular}

\subsection{Summary}

The implementation of the hybrid neural network model as an advanced parameter estimator for the first principles kinetic model developed in this research has been performed. The algorithms used to construct the hybrid neural network model were discussed, and the kinetic parameter estimates obtained were used to analyze the microscopic processes occurring at the interfaces of the $\mathrm{GaAsP} / \mathrm{GaAs}$ heterostructures. The analysis of anion exchange was greatly enhanced by the implementation of the semiempirical hybrid neural network model. The conclusions for this research and suggestions for future investigations are provided in the next chapter. 


\section{CHAPTER 7}

\section{CONCLUSIONS}

The primary mechanisms contributing to anion exchange at the interfaces of mixed anion III-V heterostructures have been investigated. The statistical experimental design technique was used in conjunction with neural network modeling to determine the effects of substrate temperature, V/III growth flux ratio, and anion exposure time on the anion exchange process for the $\mathrm{As} / \mathrm{P}$ and $\mathrm{Sb} / \mathrm{As}$ material systems. The interfacial profiles present due to anion exchange were determined; the dependence of anion exchange on atomic surface structure was established; and a hybrid neural network model capable of quantifying the effects of MBE growth processes at the interfaces of mixed anion III-V heterostructures was developed. The following results are of significance for this dissertation research:

The compositional interface profiles present for the $\mathrm{MBE}$ growth of GaInP/GaAs heterostructures were determined and correlated with specific anion exchange processes. These structures were examined based on the surface processes contributing to anion intermixing and the formation of structural defects. The $\mathrm{P}_{2} / \mathrm{GaAs}_{4}$ and $\mathrm{As}_{2} / \mathrm{GaInP}$ exchange processes were found to exhibit surface roughening during anion exposure, resulting in considerable interface degradation. The compositional interface profiles for the $\mathrm{GaAsSb} / \mathrm{GaAs}$ and $\mathrm{GaSbAs} / \mathrm{GaSb}$ heterostructures were determined using HRXRD dynamical simulations. The primary surface process contributing to anion intermixing for $\mathrm{As}_{4}$-exposed $\mathrm{GaSb}$ was determined to be anion exchange, while surface segregation dominated the anion intermixing for $\mathrm{Sb}_{2}$-exposed GaAs. 
The significance of atomic surface structure for understanding the anion exchange process has been established through the analysis of $\mathrm{GaAsP} / \mathrm{GaAs}$ interfaces. The phosphorus composition at these interfaces was determined using HRXRD and found to have a strong non-linear dependence on the substrate temperature. A neural network model was constructed to characterize the phosphorus composition, and the results were used, in conjunction with RHEED analysis, to determine the dependence of anion exchange on the MBE growth conditions. It was found that phosphorus incorporation increases as the atomic surface structure transitions from the GaAs $(001)-\mathrm{c}(4 \times 4)$ surface to the $\gamma, \beta$, and $\alpha$ GaAs $(001)-(2 \times 4)$ surface phases, with the $\alpha$ phase having the largest phosphorus composition. The investigation revealed that the anion exchange process depends primarily on the atomic surface structure present at the interface, and the Asstabilizing flux determines only the substrate temperature range for which the atomic surface structures are present.

The kinetic model developed for this research effort quantitatively describes the contributions of the microscopic processes of anion exchange, surface desorption, diffusion, and surface segregation and can be used to determine the composition of the dissimilar anion at the interfaces of mixed anion heterostructures. The implementation of the hybrid neural network model as an advanced parameter estimator for the first principles kinetic model was performed, and the kinetic parameter estimates obtained were used to analyze the microscopic processes occurring at the interfaces of the GaAsP/GaAs heterostructures. These results confirmed the dependence of anion exchange on atomic surface structure and indicated that diffusion was the dominant process occurring for these mixed anion structures. 


\section{CHAPTER 8}

\section{FUTURE RESEARCH}

The objective for the proposal of anion exchange surface mechanisms is to explore methods for applying the results obtained from the investigation of anion exchange to understanding the surface dynamics occurring at mixed anion interfaces. The hybrid neural network model results obtained for this research are essential to understanding the underlying kinetics of the microscopic processes occurring at the interfaces of mixed anion materials. These results, in conjunction with characterization data from RHEED, STM, and XPS, can be used to perform a more fundamental analysis of anion exchange. The determination of surface mechanisms involves studying the surface dynamics occurring during the transition of a group- $\mathrm{V}$ stabilized reconstructed surface to a dissimilar anion reconstructed surface. The electron counting model and other techniques can be used to narrow the possible surface mechanisms and determine if stable intermediate atomic surface structures exist.

The dependence of anion exchange on atomic surface structure indicates that different surface mechanisms occur for different initial atomic surface structures. This would account for both the various modes of anion exchange and the distinct MBE growth conditions for which anion exchange is inhibited. In order to begin formulating the relationships between microscopic processes and surface dynamics, the technique used in Section 2.2.4 can be used to establish theories related to the probabilistic behavior of dissimilar group- $\mathrm{V}$ atoms on a binary surface alloy in terms of a unit cell evolving from the binary alloys atomic surface structure to an anion inter-mixed surface structure. 


\section{APPENDIX A}

\section{STM IMAGES AND ATOMIC SURFACE MODELS}
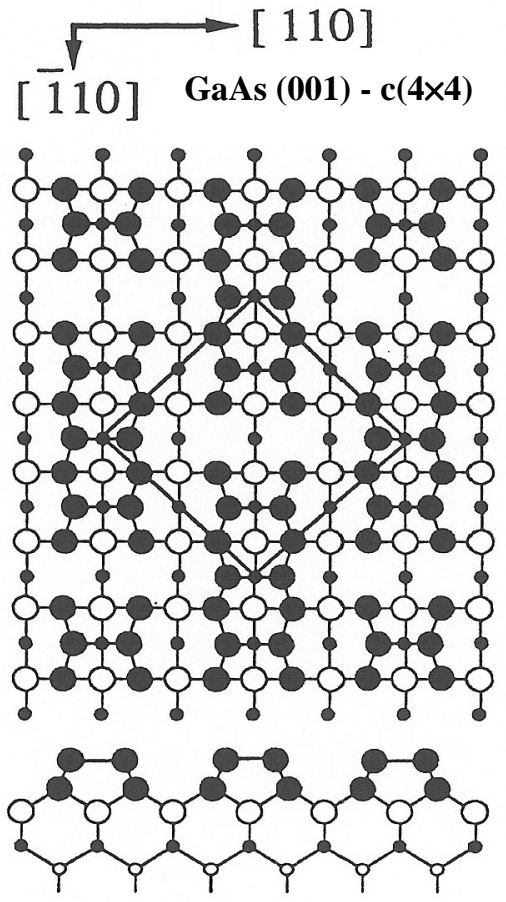
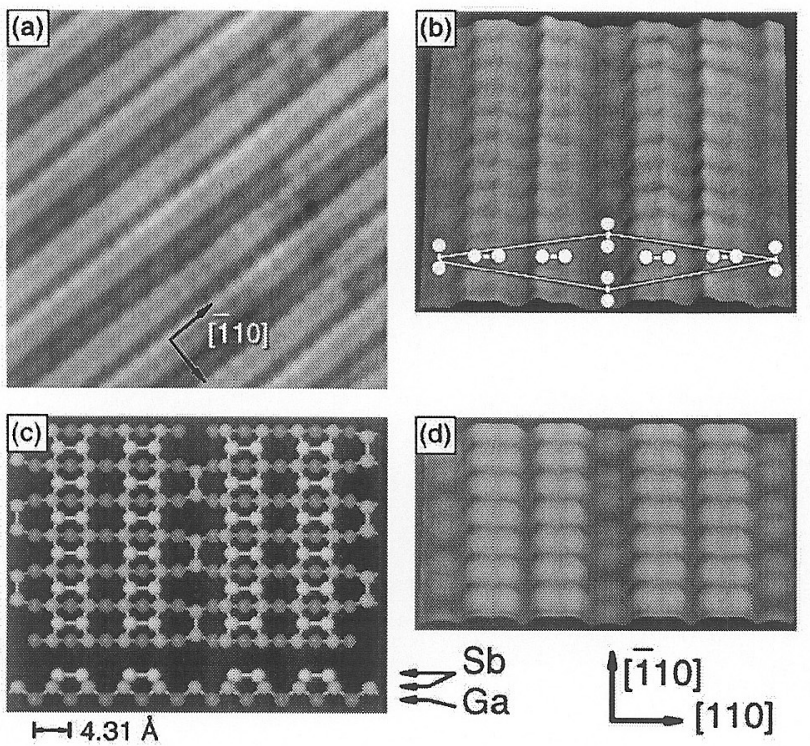

Filled-state STM images of $\mathrm{GaSb}(001)-(1 \times 5)$ acquired at $1.8 \mathrm{~V}$ : (a) $80 \AA \times 80 \AA$, (b) $48 \AA \times 48 \AA$. The lower dimers give the surface a $2 \times$ periodicity along [110]; a $c(2 \times 10)$ unit cell is indicated. Note that the slight asymmetry of the right dimer row of each row pair is a feedback artifact resulting from the large gain required to resolve the internal structure of the dimers. (c) Proposed $c(2 \times 10)$ structural model for this surface. (d) Simulated STM image of this structure calculated for filled states within $1.8 \mathrm{eV}$ of the Fermi level. 

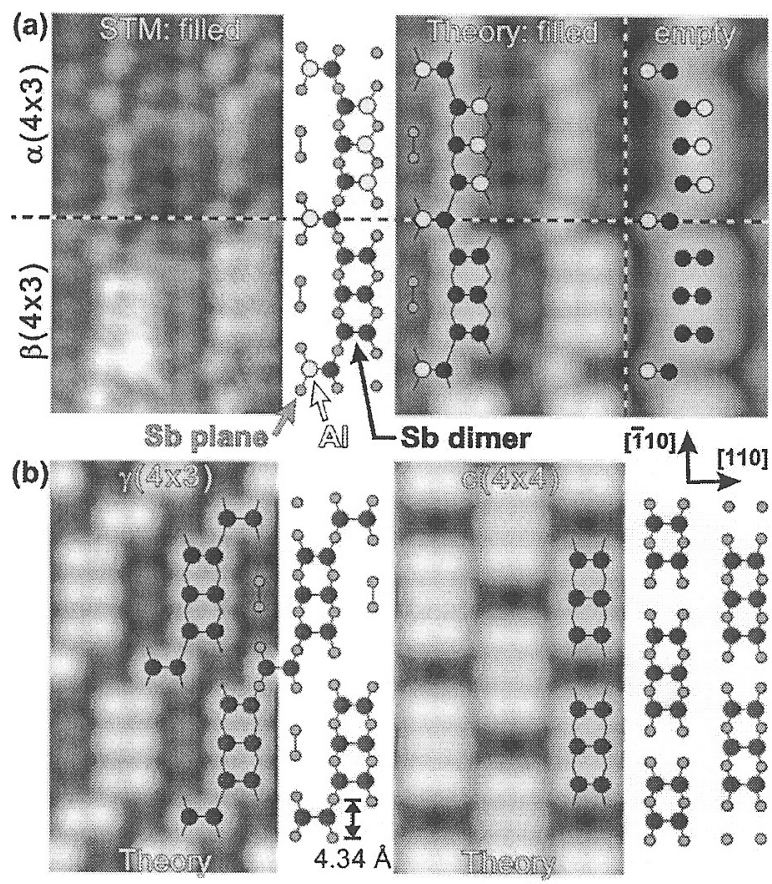

Structural models and atomic-scale constant-current images of the stable Sb-rich AlSb(001) reconstructions. (a) A comparison of the experimental (left) and simulated (right) filled-state STM images for $\alpha(4 \times 3)$ (top) and $\beta(4 \times 3)$ (bottom). Simulated empty-state images are also shown on the right [compare with the insets in Figs. 1(a) and 1(c)]. (b) Simulated images of the $\gamma(4 \times 3)$ and $c(4 \times 4)$ phases [compare with Fig. 1(e)].
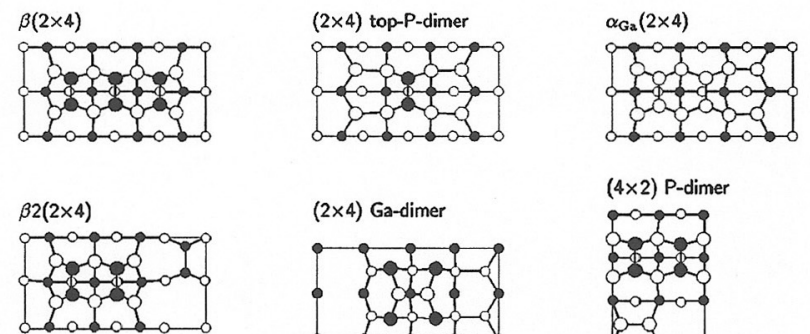

(2x4) Ga-dimer

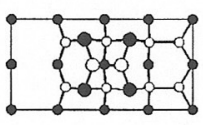

$\alpha(2 \times 4)$
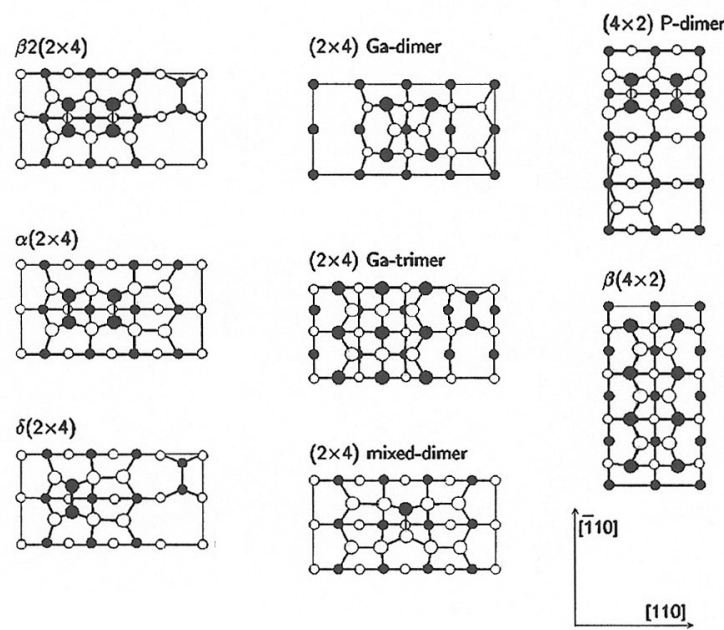

Top view of relaxed $\mathrm{GaP}(001)(2 \times 4)$ and $(4 \times 2)$ surface reconstruction models. Empty (filled) circles represent $\mathrm{Ga}(\mathrm{P})$ atoms. Large (small) symbols indicate positions in the first and second (third and fourth) atomic layers. $(2 \times 4)$ and $(4 \times 2)$ reconstructions are ordered by increasing $\mathrm{Ga}$ coverage. 


\section{APPENDIX B}

\section{RHEED AND TEM ANALYSIS}

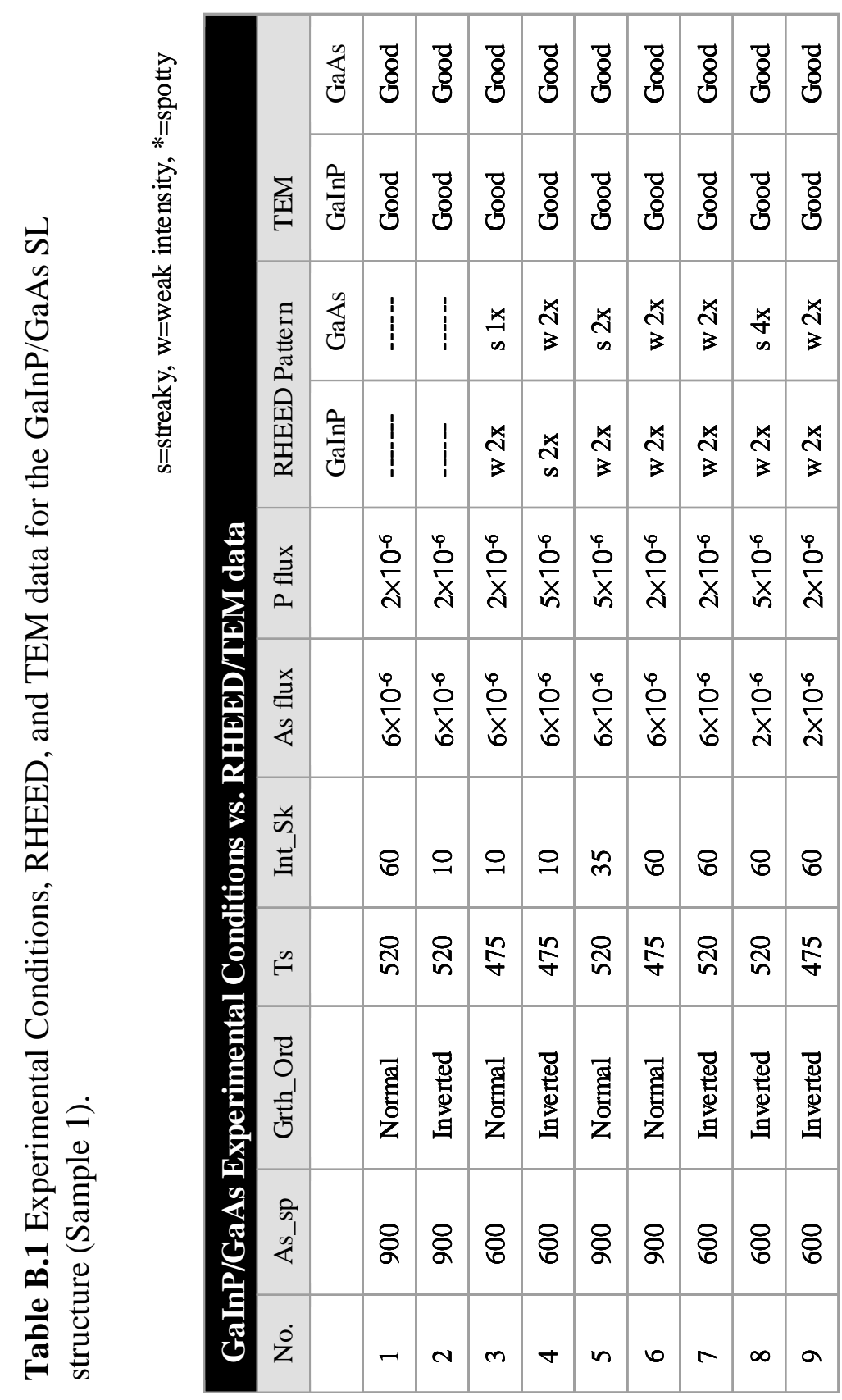




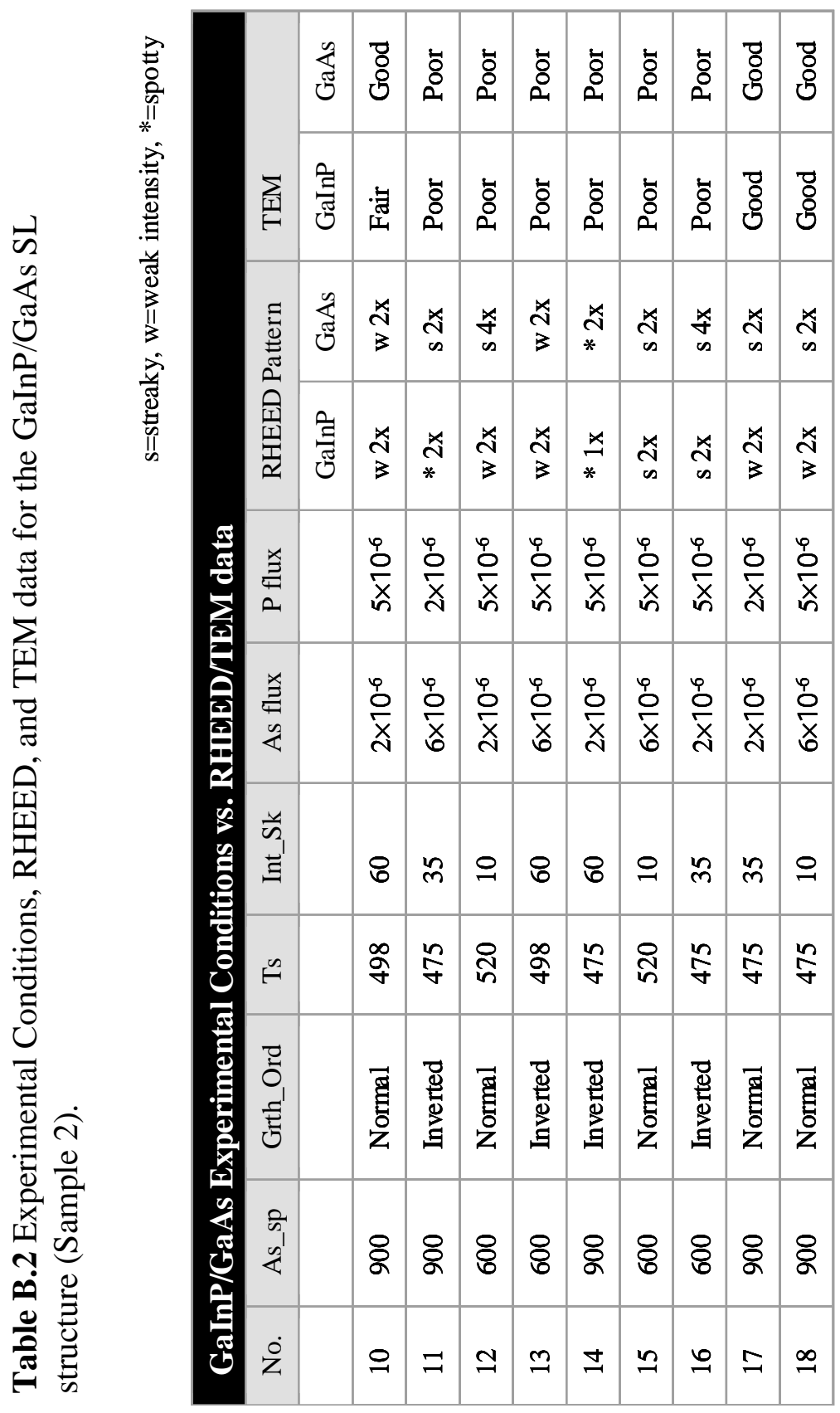




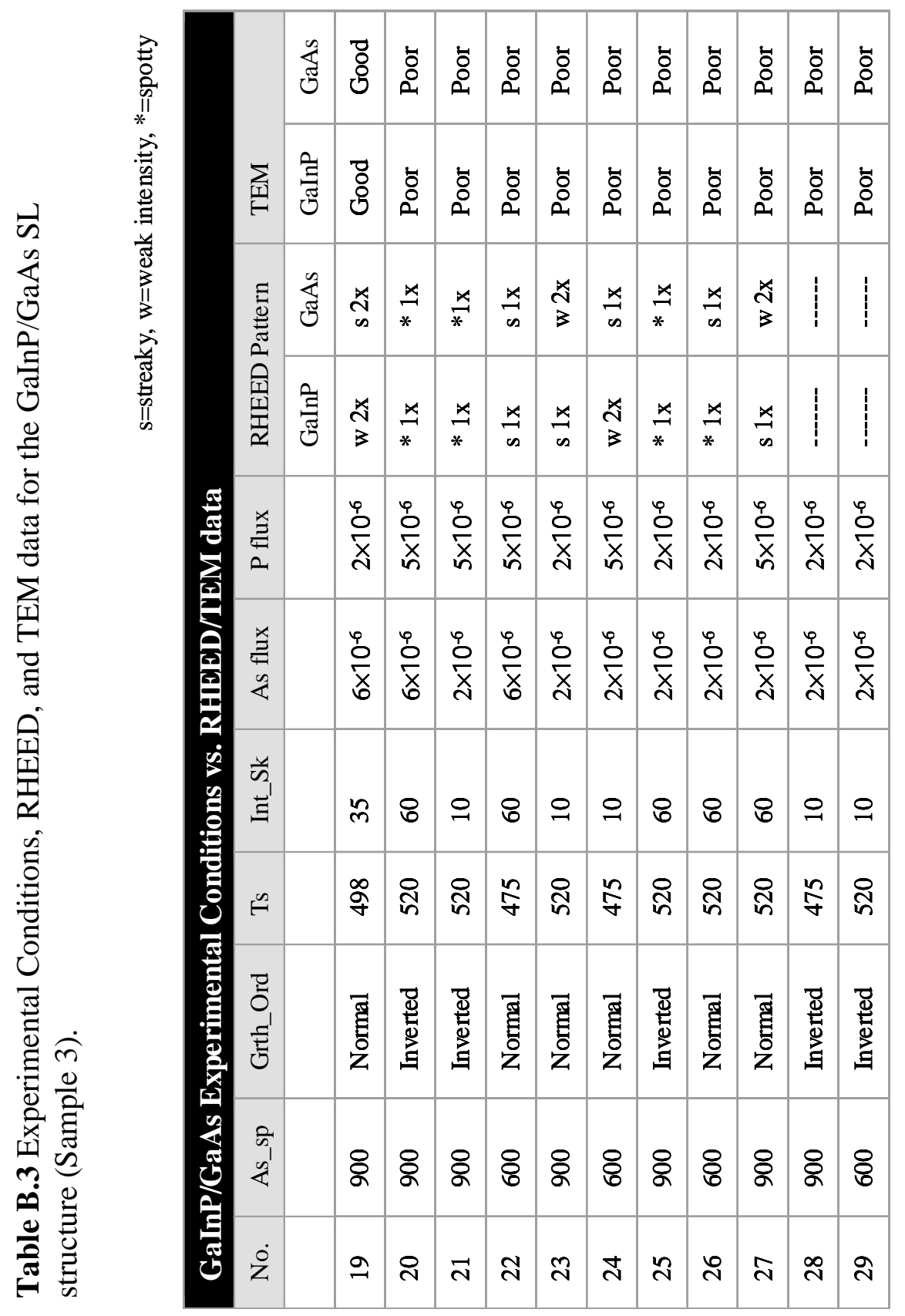




\section{APPENDIX C}

\section{RHEED CHARACTERISTIC ABSENCES}
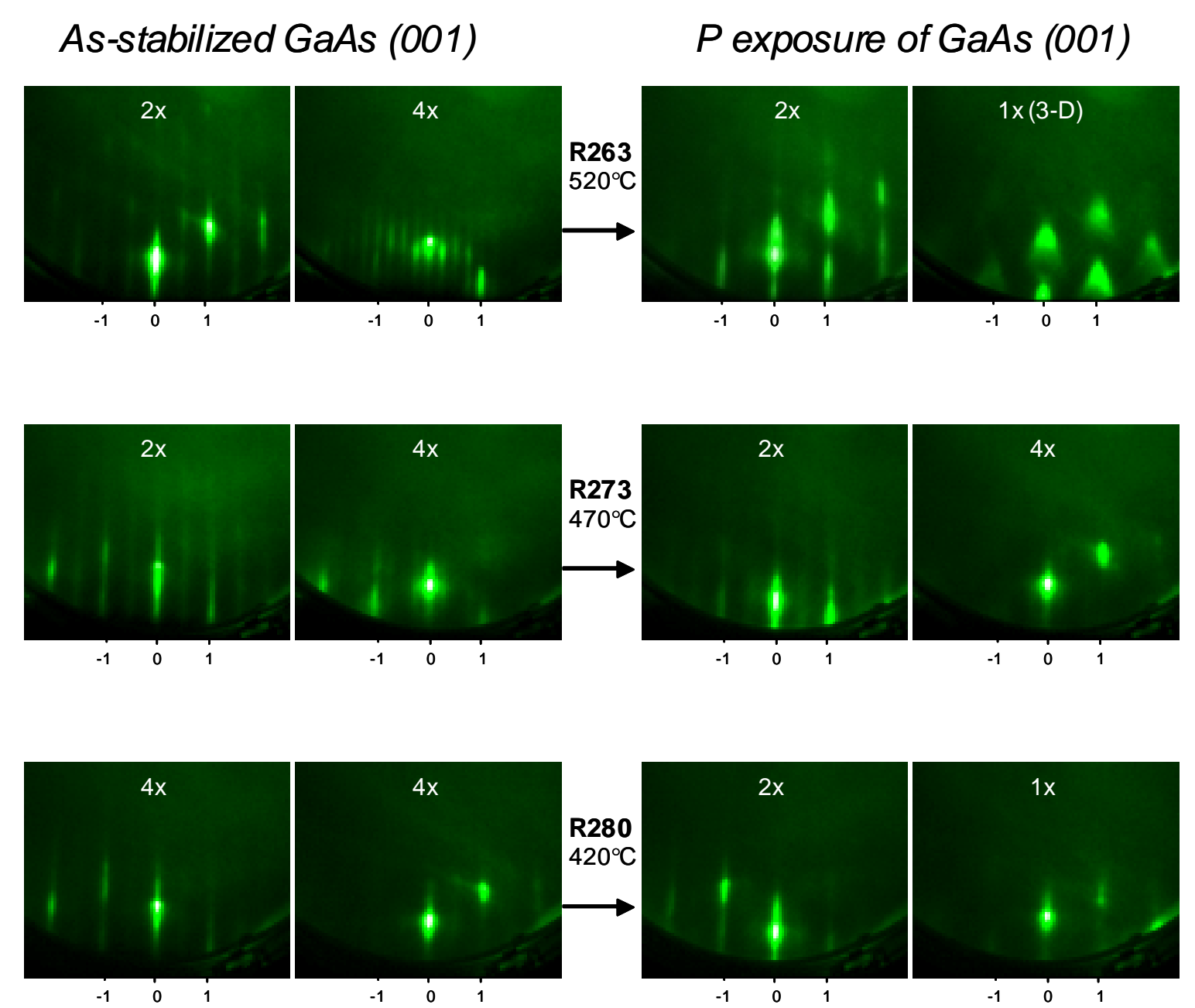

Figure C.1 RHEED data obtained during the $\mathrm{P}_{2}$ exposure of GaAs (001) surfaces. 


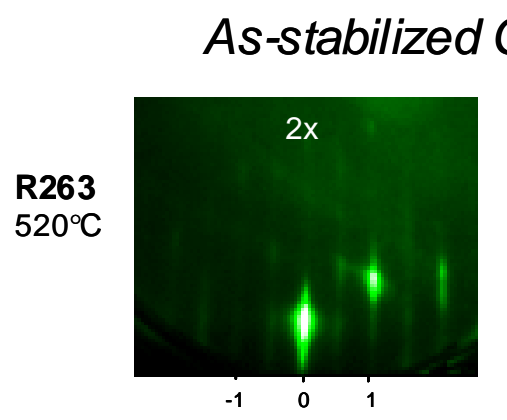

GaAs (001)
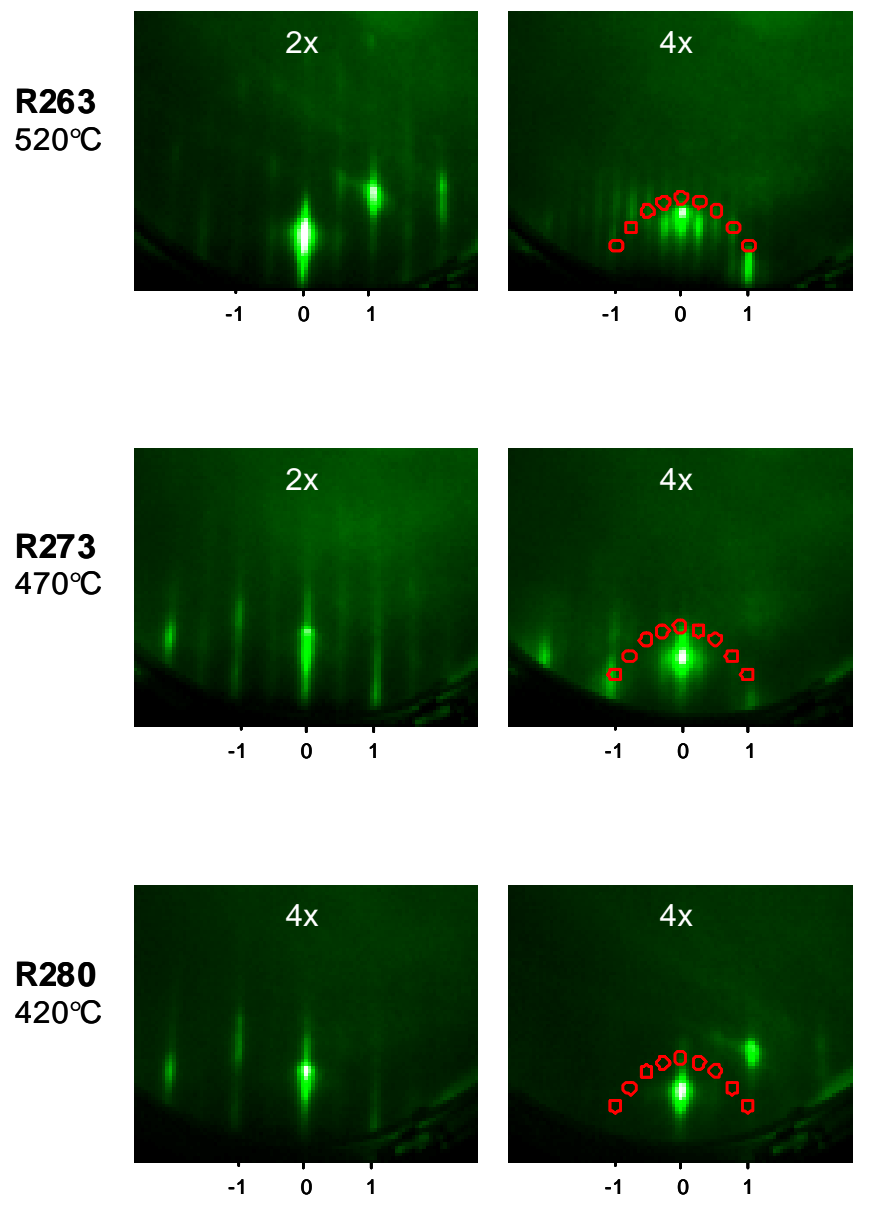

\section{Surface Structure}

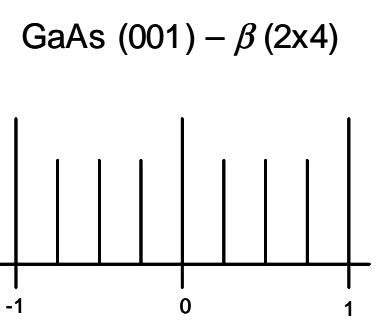

GaAs $(001)-\gamma(2 \times 4)$

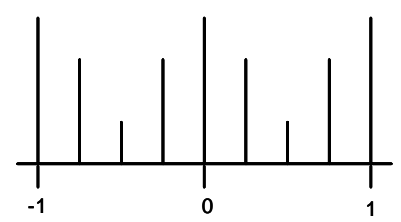

GaAs $(001)-c(4 \times 4)$

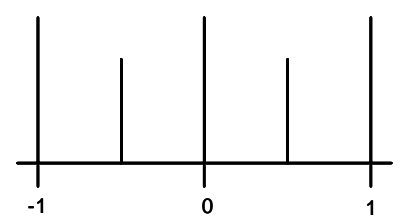

Figure C.2 Determination of atomic surface structure using the RHEED fractional order characterization technique. 


\section{APPENDIX D}

\section{OBJECT ORIENTED NEURAL NETWORK SIMULATOR}

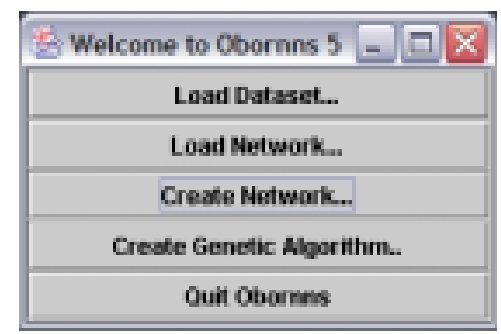

Figure D.1 The main control panel for ObOrNNS version 5.

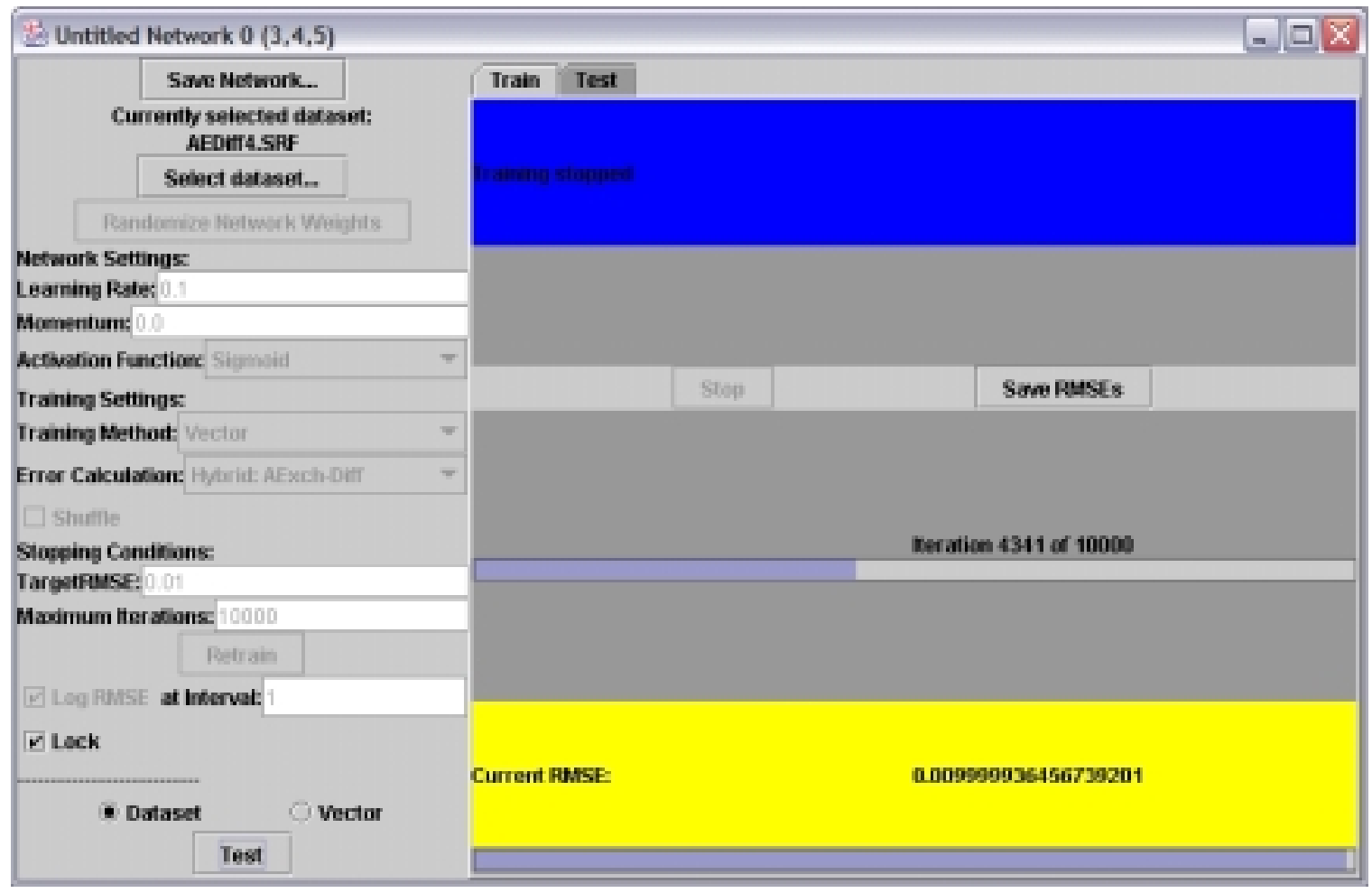

Figure D.2 The network training interface for ObOrNNS version 5. 


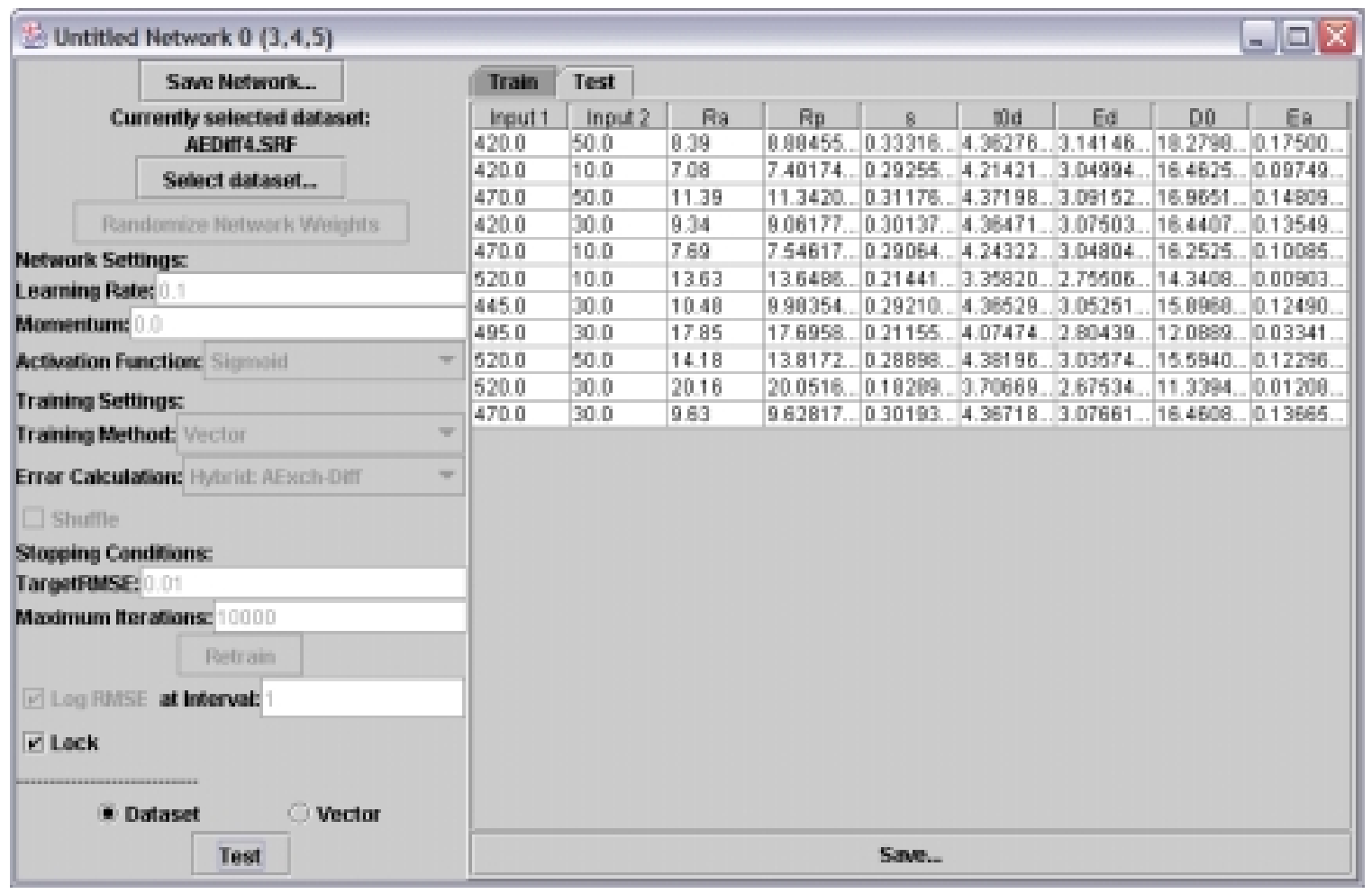

Figure D.3 The network testing interface for ObOrNNS version 5. 


\section{REFERENCES}

1. A. Y. Cho, Appl. Phys. Lett. 19, 467 (1971).

2. L. L. Chang, L. Esaki, W. E. Howard, R. Ludeke, J. Vac. Sci. Technol. 10, 11 (1973).

3. A. S. Brown, "Molecular Beam Epitaxy of III-V Materials including Migrationenhanced Epitaxy," The Encyclopedia of Advanced Materials, ed. R. Cahn, New York, New York: Pergamon Press, 1623-1629 (1990).

4. C. T. Foxon, J. Cryst. Growth 251, 1-8 (2003).

5. A. Y. Cho, D. L. Sivco, H. M. Ng, C. Gmachl, A. Tredicucci, A. L. Hutchinson, S. N. G. Chu, F. Capasso, J. Cryst. Growth 227/228, 1-7 (2001).

6. R. F. C. Farrow, J. Phys. D 67, L121 (1974).

7. A. Y. Cho, J. R. Arther, Prog. Solid State Chem. 10, 157 (1975).

8. M. A. Herman, H. Sitter, Molecular Beam Epitaxy Fundamentals and Current Status, New York, New York: Springer-Verlag (1989).

9. J. M. Blakely, Introduction to the Properties of Crystal Surfaces, New York, New York: Pergamon Press (1979).

10. C. T. Foxon, B. A. Joyce, Surf. Sci. 64, 293 (1977).

11. C. T. Foxon, B. A. Joyce, Surf. Sci. 50, 434 (1975).

12. B. G. Streetman, Solid State Electronic Devices, Englewood Cliffs, New Jersey: Prentice Hall (1990).

13. L. Esaki, R. Tsu, IBM J. Rese. Devel. 14, 61 (1970).

14. R. Dingle, H. L. Storrmer, A. C. Gossard, and W. Wiegmann, Appl. Phys. Lett. 37, 805 (1978).

15. Z. Dobrovolskis, K. Grigoras, A. Krotkus, Appl. Phys. A, 48, 245 (1989).

16. S. V. Ivanov, P. S. Kop'ev, Antimonide Related Strained-Layer Heterostructures, ed. M. O. Manasreh, Amsterdam: Gordon and Breach, 95-170, (1997). 
17. G. Triplett, G. May, and A. Brown, "Modeling Electron Mobility in MBE-Grown InAs/AlSb Thin Films for HEMT Applications Using Neural Networks," Solid-State Electronics 46, 1519-1524 (2002).

18. J.-J. Shen, A. S. Brown, R. A. Metzger, B. Sievers, L. Bottomley, P. Eckert, and W. Carter, "Modification of quantum dot properties via surface exchange and annealing: Substrate temperature effects," J. Vac. Sci. Technol. B 16, 1326 (1998).

19. B. Bennett, B. V. Shanabrook, and M. E. Twigg, J. Appl. Phys. 85, 2157 (1999).

20. D. H. Tomich, W. C. Mitchel, P. Chow, and C. W. Tu, J. Cryst. Growth 201/202, 868 (1999).

21. A. Sato, K. Ohtani, R. Terauchi, Y. Ohno, F. Matsukura, and H. Ohno, J. Cryst. Growth 201/202, 861 (1999).

22. R. Kaspi, J. Cryst. Growth 201/202, 864 (1999).

23. Q. Xie, J. E. Van Nostrand, J. L. Brown, and C. E. Stutz, J. Appl. Phys. 86, 329 (1999).

24. A. Zangwill, Physics at Surfaces, Cambridge, New York: Cambridge University Press (1988).

25. B. A. Joyce, Rep. Prog. Phys. 48, 1637 (1985).

26. E. Bauer, Z. Krist. 110, 372 (1958).

27. Q. Xie, A. Madhukar, P. Chen, N. Kobayashi, Phys. Rev. Lett. 75, 2542 (1995).

28. Y. Q. Wang, Z. L. Wang, T. Brown, A. Brown, and G. May, "Thermodynamic Analysis of Anion Exchange during Heteroepitaxy," J. Cryst. Growth 242, 5-14 (2002).

29. Z. Zhang, M. G. Lagally, Science 276, 377 (1997).

30. J. A. Venables, "Atomic Interactions and Surface Processes in Heteroepitaxy," Thin Films: Heteroepitaxial Systems, ed. W. Liu, Singapore: World Scientific (1999).

31. M. D. Pashley, Phys. Rev. B 40, 10481 (1989).

32. H. H. Farrell, J. P. Harbison, and L. D. Peterson, J. Vac. Sci. Technol. B 5, 1482 (1987).

33. E. A. Wood, J. Appl. Phys. 4, 1305 (1964). 
34. D. J. Chadi, J. Vac. Sci. Technol. A 5, 834 (1987).

35. D. J. Frankel, C. Yu, J. P. Harbison, and H. H. Farrell, J. Vac. Sci. Technol. B 5, 1113 (1987).

36. M. D. Pashley, K. W. Haberern, W. Friday, J. M. Woodall, and P. D. Kirchner, Phys. Rev. Lett. 60, 2176 (1988).

37. I. Kamiya, D. E. Aspnes, L. T. Flores, and J. P. Harbison, Phys. Rev. B 4615894 (1992).

38. J. Falta, R. M. Tromp, M. Copel, G. D. Pettit, and P. D. Kirchenr, Phys. Rev. Lett. 69, 3068 (1992).

39. H. H. Farrell and C. J. Palmstrøm, J. Vac. Sci. Technol. B 8 (4), 903 (1990).

40. D. K. Biegelsen, R. D. Bringans, J. E. Northrup, and L. E. Swartz, Phys. Rev. B 41, $5701(1990)$.

41. J. E. Northrup, Phys. Rev. B 50, 2015 (1994).

42. W. A. Harrison, Electronic Structure and the Properties of Solids, San Francisco, California: Freeman (1980).

43. J. P. Harbison and H. H. Farrell, J. Vac. Sci. Technol. B 6, 733 (1988).

44. B. A. Joyce, "Heteroepitaxial Growth Modes and Morphologies on GaAs Surfaces," Thin Films: Heteroepitaxial Systems, ed. W. Liu, Singapore: World Scientific (1999).

45. M. Kodama and M. Kimata, J. Cryst. Growth 73, 641 (1985).

46. R. A. Swalin, Thermodynamics of Solids, Wiley, New York (1972).

47. M. Yoshitake, Y. R. Aparna, Z. Yoshihara, J. Vac, Sci. Technol. A 19, 1432 (2001).

48. RS/Discover Statistical Appendices, BBN Software Products Corporation, (1995).

49. Z. Galil, J. Kiefer, "Time and Space-Saving Computer Methods, Related to Mitchelle's DETMAX, for Finding D-Optimum Designs," Technometrics 21, 301313 (1980).

50. Z. L. Wang, Reflection electron microscopy and spectroscopy for surface analysis. New York, New York: Cambridge University Press, (1996).

51. B. W. Liang, C. W. Tu, J. Appl. Phys. 74, 255 (1993). 
52. S. Yu. Karpov, M. A. Maĭorov, Tech. Phys. Lett. 23, 38 (1997).

53. Z. Z. Bandić, T. C. McGill, R. J. Hauenstein, M. L. O’Steen, J. Vac. Sci. Technol. B 14, 2948 (1996).

54. K. K. Lee, W. A. Doolittle, A. S. Brown, G. S. May, "Using statistical experimental design to investigate the role of the initial conditions on $\mathrm{GaN}$ epitaxial films grown by molecular beam epitaxy," J. Vac. Sci. Technol. B 18, 1448 (2000)

55. C. Himmel, G. May, "Advantages of Plasma Etch Modeling Using Neural Networks Over Statistical Techniques," IEEE Trans. Semi. Manufac., 6, 103 (1993).

56. D. C. Psichogios, L. H. Ungar, AIChE 38, 1499 (1992).

57. H. C. Casey and M. B. Panish, Heterostructure Lasers part B, New York: Academic Press (1978).

58. K. Ouchi, T. Mishima, J. Cryst. Growth 209, 242 (2000).

59. J. C. Garcia, J. Cryst. Growth 127, 255 (1993).

60. J. M. Kuo, Y. K. Chen, IEEE Electron. Dev. Lett. 15, 13 (1994).

61. Y. Wang, Z. Wang, T. Brown, A. Brown, and G. May, "Configurations of Misfit Dislocations at Interfaces of Lattice-Matched GaInP/GaAs Heterostructures," Appl. Phys. Let. 77, 223-225 (2000).

62. Y. Wang, Z. Wang, T. Brown, A. Brown, and G. May, "Lateral Compositional Modulation in Lattice-Matched GaInP/GaAs Heterostructures," J. Elec. Mat. 29, 1372-1379 (2000).

63. M. Yamada, T. Anan, K. Kurihara, K. Nishi, K. Tokutome, and A. Kamei, Electron. Lett. 36, 637 (2000).

64. F. Quochi, D. Kilper, J. Cummingham, M. Dinu, and J. Shah, IEEE Photonics Technol. Lett. 13, 921 (2001).

65. O. Blum and J. Klem, IEEE Photonics Technol. Lett. 12, 771 (2000).

66. D. Bimberg, M. Grundmann, and N. Ledentsov, Quantum Dot Heterostructures, Sussex: Wiley (1999).

67. B. R. Bennet, R. Magno and B. V. Shanabrook, Appl. Phys. Lett. 68, 505 (1996).

68. R. Kaspi, W. T. Cooley, K. R. Evans, J. Cryst. Growth 173, 5 (1997). 
69. M. Wang, D. Collins, T. C. McGill, R. Grant, R. Feenstra, J. Vac. Sci. Technol. B 13, 1689 (1995).

70. P. C. Huang, S. R. Stock, A. Torabi, C. J. Summers, Advances in X-ray Analysis 33, 67 (1990).

71. P. Yashar, M. Pillai, J. Mirecki-Millunchick, and S. Barnett, J. Appl. Phys. 83, 2010 (1998).

72. J. Schmitz, J. Wagner, F. Fuchs, N. Herres, P. Koidl, and J. D. Ralston, J. Cryst. Growth 150, 858 (1994).

73. T. Brown, A. Brown, and G. May, "Anion Exchange at the Interfaces of Mixed Anion III-V Heterostructures Grown by Molecular Beam Epitaxy," J. Vac. Sci. Technol. B 20, 1771 (2002).

74. B. A. Fox, W. A. Jesser, J. Appl. Phys. 68, 2739 (1990).

75. P. F. Fewster, C. J. Curling, J. Appl. Phys. 62, 4154 (1987).

76. T. Nomura, H. Ogasawara, M. Miyao, and Hagino, J. Cryst.Growth 111, 61 (1991).

77. C. T. Foxon, J. Vac. Sci. Technol. B 1, 293 (1983).

78. J. R. Arthur and J. L. Lepore, J. Vac. Sci. Technol. 65, 45 (1969).

79. C. H. Chen, D. S. Cao, G. B. Stringfellow, J. Electron. Mater. 17, 67 (1983).

80. J. E. Cunningham, M. B. Santos, K. W. Goossen, M. D. Williams, W. Jan, Appl. Phys. Lett. 64, 2418 (1994).

81. C. T. Foxon, B. A. Joyce, M. T. Norris, J. Cryst. Growth 49, 132 (1980).

82. V. P. LaBella, D. W. Bullock, C. Emery, Z. Ding, P. M. Thibado, Appl. Phys. Lett. 79, 19 (2001).

83. Z. Nami, O. Misman, A. Erbil and G. May, "Semi-Empirical Neural Network Modeling of Metal-Organic Chemical Vapor Deposition," IEEE Trans. Semi. Manufac. 10, 288-294, (1997). 


\section{VITA}

Terence Dominic Brown was born on December 3, 1974 in Atlanta, Georgia. His desire to perform research in the fields of Science and Technology began at the Physics Department of Clark Atlanta University. He later performed DNA research at the Dolphous E. Mulligan Research Institute. Terence received his Bachelor and Master of Science degrees in Electrical Engineering from the Georgia Institute of Technology in June 1996 and September 1998, respectively.

Terence entered into the Ph.D. program in the College of Engineering at the Georgia Institute of Technology in 1998. He has worked in the fields of Microelectronics and Digital Signal Processing since 1996, conducting research and development for various initiatives. He is currently a member of the Institute of Electrical and Electronics Engineers and the Golden Key National Honor Society. His primary research interests focus on the design, growth, and investigation of compound semiconductor structures for device applications using in-situ monitoring, coupled with statistical analysis and advanced modeling techniques. 